\title{
Five-dimensional cohomological localization and squashed $q$-deformations of two-dimensional Yang-Mills theory
}

\author{
Leonardo Santilli, ${ }^{a}$ Richard J. Szabo ${ }^{b, c, d}$ and Miguel Tierz ${ }^{e, a}$ \\ ${ }^{a}$ Grupo de Física Matemática, Departamento de Matemática, Faculdade de Ciências, \\ Universidade de Lisboa, Campo Grande, Edifício C6, 1749-016 Lisboa, Portugal \\ ${ }^{b}$ Department of Mathematics, Heriot-Watt University, Colin Maclaurin Building, \\ Riccarton, Edinburgh EH14 4AS, U.K. \\ ${ }^{c}$ Maxwell Institute for Mathematical Sciences, Bayes Centre, \\ 47 Potterrow, Edinburgh EH8 9BT, U.K. \\ ${ }^{d}$ Higgs Centre for Theoretical Physics, James Clerk Maxwell Building, \\ Kings Buildings, Edinburgh EH9 3JZ, U.K. \\ e Departamento de Matemática, ISCTE - Instituto Universitário de Lisboa, \\ Avenida das Forças Armadas, 1649-026 Lisboa, Portugal \\ E-mail: lsantilli@fc.ul.pt, r.j.szabo@hw.ac.uk, mtpaz@iscte-iul.pt
}

ABSTRACT: We revisit the duality between five-dimensional supersymmetric gauge theories and deformations of two-dimensional Yang-Mills theory from a new perspective. We give a unified treatment of supersymmetric gauge theories in three and five dimensions using cohomological localization techniques and the Atiyah-Singer index theorem. We survey various known results in a unified framework and provide simplified derivations of localization formulas, as well as various extensions including the case of irregular Seifert fibrations. We describe the reductions to four-dimensional gauge theories, and give an extensive description of the dual two-dimensional Yang-Mills theory when the three-dimensional part of the geometry is a squashed three-sphere, including its extension to non-zero area, and a detailed analysis of the resulting matrix model. The squashing parameter $b$ yields a further deformation of the usual $q$-deformation of two-dimensional Yang-Mills theory, which for rational values $b^{2}=p / s$ yields a new correspondence with Chern-Simons theory on lens spaces $L(p, s)$.

Keywords: Field Theories in Higher Dimensions, Field Theories in Lower Dimensions, Supersymmetric Gauge Theory, Differential and Algebraic Geometry

ARXiv EPrint: 2003.09411 


\section{Contents}

1 Introduction 1

2 Preliminaries on superconformal field theories and localization 3

2.1 Squashed geometry and six-dimensional superconformal field theories 3

2.2 Reductions to two and three dimensions 5

$\begin{array}{lll}2.3 & \text { Basics of cohomological localization } & 7\end{array}$

2.4 Localization of $\mathcal{N}=1$ gauge theories on Seifert manifolds 8

$3 \mathcal{N}=2$ cohomological gauge theories in three dimensions 12

3.1 Supersymmetric Yang-Mills theory and its cohomological formulation 12

$\begin{array}{lll}3.2 & \text { One-loop determinant of the vector multiplet in a regular background } \quad 15\end{array}$

3.3 One-loop determinant of the vector multiplet in an irregular background 17

$\begin{array}{ll}3.4 & \text { One-loop determinant of a hypermultiplet } \\ 3.5 & 17\end{array}$

$\begin{array}{ll}3.5 & \text { Applications of the cohomological localization formulas } \\ & 18\end{array}$

$\begin{array}{ll}3.6 & \text { Extension to } S^{1} \times C_{g}\end{array} 21$

$4 \mathcal{N}=1$ cohomological gauge theories in five dimensions $\quad 22$

4.1 Supersymmetric Yang-Mills theory and its cohomological formulation 23

4.2 One-loop determinant of the vector multiplet in a regular background 26

4.3 One-loop determinant of the vector multiplet in an irregular background 28

4.4 One-loop determinant of a hypermultiplet 30

$\begin{array}{lll}4.5 & \text { Perturbative partition functions } & 31\end{array}$

4.6 Contact instantons and their pushdown to four dimensions 32

$\begin{array}{lll}4.7 & \text { Borel summability } & 35\end{array}$

4.8 Extension to $S^{1} \times C_{g} \times \Sigma_{h}$

$5 \quad q$-deformed Yang-Mills theories from cohomological localization $\quad 38$

$\begin{array}{lll}5.1 & \text { Localization on } S^{3} \times \Sigma_{h} & 38\end{array}$

$\begin{array}{lll}5.2 & \text { Localization on squashed } S_{b}^{3} \times \Sigma_{h} & 42\end{array}$

5.3 Localization on ellipsoid $S_{b}^{3} \times \Sigma_{h} \quad 42$

5.4 The matrix model 44

5.5 Localization from seven dimensions $\quad 50$

$\begin{array}{ll}\text { A Spinor conventions } & 53\end{array}$

$\begin{array}{ll}\text { B Squashed three-spheres } & 54\end{array}$

$\begin{array}{ll}\text { C Sasaki-Einstein five-manifolds } & 56\end{array}$ 


\section{Introduction}

Supersymmetric gauge theories in five and six dimensions have undergone a surge of extensive and diverse investigations in recent years. They have played critical roles in the understanding of the strong coupling dynamics of quantum field theory, of M-theory where they can be engineered, and of various problems in geometry. Their compactifications generate many interesting theories in lower dimensions, which can be used to elucidate novel features of lower-dimensional quantum field theories from a geometric perspective.

Five-dimensional gauge theories with $\mathcal{N}=1$ supersymmetry can be engineered from compactifications of M-theory on Calabi-Yau threefolds [1-3], which are related to the embedding of topological string theory into M-theory [4]. In six dimensions the worldvolume theory of coincident M5-branes is a six-dimensional $\mathcal{N}=(2,0)$ superconformal field theory. Twisted compactification of this worldvolume theory on an $n$-punctured Riemann surface $\Sigma_{h, n}$ of genus $h$ generically engineers an $\mathcal{N}=2$ superconformal field theory of class $\mathcal{S}$ in four dimensions [5,6], which upon further twisted compactification on a circle $S^{1}$ in the Schur limit is conjecturally equivalent to two-dimensional $q$-deformed Yang-Mills theory on $\Sigma_{h, n}$ in the zero area limit [7]. Because of the lack of a Lagrangian description of this six-dimensional theory, its twisted partition function is most easily computed by dimensionally reducing on a circle of radius $\beta$ and computing the twisted partition function of five-dimensional $\mathcal{N}=2$ supersymmetric Yang-Mills theory with gauge coupling $g_{\mathrm{YM}}^{2}=2 \pi \beta[8,9]$; the duality with two-dimensional $q$-deformed Yang-Mills theory in this five-dimensional setting was checked by [10] using explicit supersymmetric localization techniques. From the four-dimensional perspective, the class $\mathcal{S}$ theory is then equivalent to a three-dimensional theory on a certain squashed deformation $M_{b}$ of the three-dimensional part of the compactification in six dimensions. In this paper we mostly focus on the round three-sphere $S^{3}$ and its squashed deformations $S_{b}^{3}$.

These dualities have by now been extensively discussed. The purpose of the present paper is to survey and reinvestigate these correspondences from a new and more detailed perspective; we give extensive pointers to and comparisons with relevant previous works on the subject as we go along. The main technique which we exploit in this paper is cohomological localization, as pioneered by Källén for three-dimensional theories in [11], and subsequently extended to five dimensions by Källén, Qiu and Zabzine in [12, 13]. This enables a unified treatment of supersymmetric gauge theories in three and five dimensions using solely topological techniques based on the Atiyah-Singer index theorem, which extends and simplifies previous treatments based on supersymmetric localization; this method is ultimately one of the main messages of the present work. Compared to previous work on the subject, we study full topologically twisted theories, instead of partially twisted theories, with which we further extend general results in the literature on cohomological localization. Our simplified treatment also unavoidably comes with some limitations, and we shall extensively discuss which backgrounds do not fit into our localization framework.

In three dimensions we provide different simplified derivations of some known scattered results, treated in a unified framework. For example, the index theory calculations of localization on $S_{b}^{3}$ in [14] uses a procedure which is different from that of [11], and their Lie 
derivative appearing in the square of the supercharge is not along the Reeb vector field. We will explain this point thoroughly in this paper. When the supersymmetry transformations of [14, appendix B] are put into the cohomological form of [11], index theorem calculations can be applied to obtain the same results in a more economical way. Generally, our procedure of topological twisting rigorously justifies the localization calculations we employ at the field theory level; in particular, it justifies the usage of the index formula and related techniques from $[12,13]$.

In five dimensions the core of our work starts, where the reader versed in localization techniques may begin. We adapt the formalism of $[12,13]$ to reobtain some known results in a different way using a twisted gauge theory approach and also extend some general results (see in particular our localization formulas (4.23) and (4.32)). In particular, we rederive the results of $[15,16]$ through the Atiyah-Singer index theorem, first in the case of $S^{3} \times \Sigma_{h, 0}$ (which for $h=0$ is a relatively straightforward adaptation from the literature), and then extending it to $S_{b}^{3} \times \Sigma_{h, 0}$. We also describe the pushdown of these theories to the horizontal four-dimensional part of the geometry, which was justified in [16] only for $h=0$, and is different from [13]. We study the resulting theory in detail and describe its precise relation to $q$-deformed Yang-Mills theory on $\Sigma_{h, 0}$; we improve on various results in the literature (with some overlap with [16]), for example determining the two-dimensional theory at non-zero area, and pay more attention to the underlying matrix model, which has not been previously considered. For non-trivial squashing parameters $b \neq 1$, the resulting two-dimensional gauge theory is new and we refer to it as a squashed $q$-deformation of Yang-Mills theory on $\Sigma_{h, 0}$. When $b^{2} \in \mathbb{Q}$, we show that this theory is closely related to the $q$-deformations of Yang-Mills theory considered in [17] in a completely different setting, which in turn is related to three-dimensional Chern-Simons theory on general lens spaces $L(p, s)$.

The outline of the remainder of this paper is as follows. We have endeavoured throughout to give a relatively self-contained presentation, while glossing over some well-known technical aspects for which we refer to the pertinent literature; thus some of the earlier parts of this paper are somewhat expository in nature. We begin in section 2 by giving a more detailed introduction and background to the setting discussed briefly above, summarising the geometric settings and classifications, techniques and notation that will be used throughout this paper.

Section 3 is dedicated to the three-dimensional case, wherein we review the ideas behind Källén's cohomological localization technique, and explain how they are modified on squashed manifolds. We discuss the construction of the cohomological gauge theory, the computation of the one-loop determinants for the vector multiplet on the two possible classes of Seifert three-manifolds admitting $\mathcal{N}=2$ supersymmetry, and the computation of the hypermultiplet one-loop determinants. We describe several explicit applications of our localization formulas.

Section 4 presents the five-dimensional analog of our considerations from section 3 . We describe the cohomological field theory and derive the one-loop determinants of the vector multiplet in the two classes of Seifert five-manifolds admitting $\mathcal{N}=1$ supersymmetry. We describe explicit applications of our localization formulas, and we briefly address the proof 
of Borel summability of the perturbative partition functions. We also explain in detail the relationship with a four-dimensional theory.

Section 5 explains the relation between our five-dimensional cohomological field theories and two-dimensional Yang-Mills theory. We derive the standard $q$-deformation of Yang-Mills theory on $\Sigma_{h, 0}$ through a localization calculation on $S^{3} \times \Sigma_{h, 0}$, and subsequently extend these considerations to the squashed deformations $S_{b}^{3} \times \Sigma_{h, 0}$ where we obtain a new two-parameter deformation. We investigate the matrix model in detail and derive a new correspondence with Chern-Simons gauge theory on lens spaces $L(p, s)$. We conclude by briefly addressing how to obtain more general deformations of two-dimensional Yang-Mills theory through localization calculations in higher dimensions.

Three appendices at the end of the paper provide various technical details complementing some of the analysis in the main text. Appendix A summarises our conventions and notation for spinors, which are adapted to treat the three-dimensional and five-dimensional cases, as well as the vector multiplets and matter hypermultiplets, in a unified way. Appendix B provides mathematical details of the different types of squashings of spheres that preserve $\mathcal{N}=2$ supersymmetry in three dimensions. Appendix $\mathrm{C}$ provides some mathematical details on Sasaki-Einstein manifolds, which preserve $\mathcal{N}=1$ supersymmetry in five dimensions, and we briefly review the formalism of [18] to better explain why our results appear to be so different from those of $[18,19]$.

\section{Preliminaries on superconformal field theories and localization}

In this preliminary section we collect the relevant background material that will be used throughout this paper. We begin with a discussion of superconformal field theories in six dimensions, which gives one of the primary motivations behind the present investigations. We discuss how certain compactifications of these theories on a Riemann surface suggest a duality between four-dimensional superconformal theories and the standard $q$-deformation of two-dimensional Yang-Mills theory. The purpose of this paper is to investigate in detail how this duality is modified in the case where the three-dimensional part of the compactification is a squashed geometry, and we describe how cohomological localization techniques for superconformal field theories on Seifert manifolds will be applied to investigate the correspondence.

\subsection{Squashed geometry and six-dimensional superconformal field theories}

Consider the six-dimensional superconformal $(2,0)$ theory with gauge group $G$ of ADE type on a twisted compactification of the form

$$
M_{6}=S^{1} \times S^{3} \times \Sigma_{h}
$$

where $S^{3}$ is the standard round three-sphere and $\Sigma_{h}$ is a compact oriented Riemann surface of genus $h$ without boundaries. This setup dictates a remarkable duality: the correlators of a certain two-dimensional topological quantum field theory on $\Sigma_{h}$ compute the partition function of a four-dimensional $\mathcal{N}=2$ field theory of class $\mathcal{S}$ on $S^{1} \times S^{3}$, which is the 
superconformal index $[20]$

$$
\mathcal{I}(u, q, t)=\operatorname{Tr}(-1)^{\mathrm{F}}\left(\frac{t}{u q}\right)^{\mathrm{r}} u^{\mathrm{J}_{+}} q^{\mathrm{J}_{-}} t^{\mathrm{R}},
$$

where $J_{ \pm}$are the rotation generators in the two orthogonal planes constructed from the Cartan generators of the Lorentz $\mathrm{SU}(2)_{\mathrm{L}} \times \mathrm{SU}(2)_{\mathrm{R}}$ isometry group of $S^{3}$, the operator $r$ is the $\mathrm{U}(1)_{r}$ generator, and $\mathrm{R}$ is the $\mathrm{SU}(2)_{R}$ generator of $\mathrm{R}$-symmetries. The superconformal index of four-dimensional $\mathcal{N}=2$ superconformal field theories was originally introduced in [21, $22]$, and it is a highly non-trivial function of the three superconformal fugacities $(u, q, t)$.

However, this duality is difficult to test because our current understanding of the sixdimensional $(2,0)$ theory is rather incomplete. Instead, we dimensionally reduce over $S^{1}$, which yields five-dimensional supersymmetric Yang-Mills theory. The Yang-Mills coupling in five dimensions has dimensions of length, $g_{\mathrm{YM}}^{2}=2 \pi \beta$, where $\beta$ is the radius of $S^{1}$. The dimensional reduction over $S^{1}$ of the supersymmetric partition function on $S^{1} \times S^{3}$ is achieved by assigning scaled chemical potentials to the fugacities according to

$$
q=\mathrm{e}^{2 \pi \mathrm{i} \beta \epsilon_{1}}, \quad t=\mathrm{e}^{2 \pi \mathrm{i} \beta \epsilon_{2}} \quad \text { and } \quad u=\mathrm{e}^{2 \pi \mathrm{i} \beta},
$$

and taking the limit $\beta \rightarrow 0$. Then the four-dimensional index becomes a three-dimensional ellipsoidal partition function [23-25], i.e. the partition function on the squashed sphere $S_{b}^{3}$ with squashing parameter

$$
b=\sqrt{\frac{\epsilon_{1}}{\epsilon_{2}}} .
$$

This deformation of the three-sphere of radius $r=\sqrt{\epsilon_{1} \epsilon_{2}}$ can be parametrized by the ellipsoid in $\mathbb{C}^{2}$ defined by

$$
b^{2}\left|z_{1}\right|^{2}+b^{-2}\left|z_{2}\right|^{2}=r^{2},
$$

which has isometry group $\mathrm{U}(1) \times \mathrm{U}(1)$. In this setting the deformation parameters $\left(\epsilon_{1}, \epsilon_{2}\right)$ can also be interpreted as equivariant parameters of the $\Omega$-background [26], which are the holonomies of the twisted M-theory compactification defined by an $S^{1}$-bundle over $M_{6} \times \mathrm{TN}$ realised as the quotient of $\mathbb{R} \times M_{6} \times \mathrm{TN}$ by the $\mathbb{Z}$-action

$$
n \cdot\left(\tau, x, z_{1}, z_{2}\right)=\left(\tau+2 \pi \beta n, x, q^{n} z_{1}, t^{-n} z_{2}\right),
$$

where $n \in \mathbb{Z}, \tau \in \mathbb{R}, x \in M_{6}$ and $\left(z_{1}, z_{2}\right) \in \mathbb{C}^{2}$ are local coordinates on the Taub-NUT space TN.

Hence we consider supersymmetric Yang-Mills theory on the five-manifold

$$
M_{5}=S_{b}^{3} \times \Sigma_{h}
$$

This theory sits in the infrared of a renormalization group flow triggered by a relevant deformation of a five-dimensional superconformal field theory in the ultraviolet [27]. For the ellipsoid $S_{b}^{3}$ [28] described above, the five-dimensional theory has four supercharges and can alternatively be taken to directly descend from the $\mathcal{N}=(1,0)$ superconformal field theory on $S^{1} \times S_{b}^{3} \times \Sigma_{h}$, with only one chiral Killing spinor in six dimensions. On the 
other hand, from the present perspective it is more natural to use the squashed sphere $S_{b}^{3}$ of [29] (see appendix B for details), for which the five-dimensional theory descends from twisted compactification of the $\mathcal{N}=(2,0)$ superconformal field theory on $S^{1} \times S^{3} \times \Sigma_{h}$ with round $S^{3}$. The geometric meaning of the squashing parameter $b$ is different in the two cases: $b>0$ for the ellipsoid, while $b \in \mathbb{C}$ with $|b|=1$ for the squashed sphere. From the point of view of the matrix ensemble we will find in section 5.4, the natural choice would be $b>0$. In section 5 we consider both cases. We will find that, regardless of what choice we make for $S_{b}^{3}$, the partition function is the same and is a holomorphic function of $b$ in the punctured complex plane $\mathbb{C} \backslash\{0\}$, so we may start with either the squashed sphere or the ellipsoid and then analytically continue the result.

\subsection{Reductions to two and three dimensions}

By a localization calculation over the squashed sphere $S_{b}^{3}$, we will identify the partition function of the two-dimensional gauge theory dual on $\Sigma_{h}$ of the $\mathcal{N}=2$ theory of class $\mathcal{S}$. In this paper we are exclusively interested in the slice of the superconformal fugacity space defined by the Schur limit $u=0, q=t$ of the superconformal index which is the Schur index

$$
\mathcal{I}(q)=\operatorname{Tr}(-1)^{\mathrm{F}} q^{\mathrm{J}_{-}+\mathrm{R}}
$$

where the trace is now restricted to states with $\mathrm{U}(1)_{r}$ charge $r=J_{+}$. In this case the index was computed in [7, 30] from a topological quantum field theory on the Riemann surface $\Sigma_{h}$, which can be identified with the zero area limit $\operatorname{vol}\left(\Sigma_{h}\right)=0$ of the usual $q$-deformed two-dimensional Yang-Mills theory; the duality with this two-dimensional gauge theory is confirmed in [10] by an explicit localization computation on $S^{3} \times \Sigma_{h}$ (i.e. for $b=1$ ). The $q$-deformed Yang-Mills theory is not topological when $\operatorname{vol}\left(\Sigma_{h}\right) \neq 0$, but it still has a natural class $\mathcal{S}$ theory interpretation as the supersymmetric partition function of the $(2,0)$ theory on $S^{1} \times S^{3} \times \Sigma_{h}$ where the area of the ultraviolet curve $\Sigma_{h}$ is kept finite [31]. We shall study this proposal explicitly via a localization calculation on the five-manifold $S_{b}^{3} \times \Sigma_{h}$. The two-dimensional theory we find is a further deformation by the squashing parameter $b$, that we call 'squashed' $q$-deformed Yang-Mills theory. For later use, let us now briefly review the standard $q$-deformation of two-dimensional Yang-Mills theory.

Let $\mathfrak{g}$ be the Lie algebra of a connected Lie group $G$. Let $\triangle$ be the root system of $\mathfrak{g}$ and $\triangle_{+}$the system of positive roots; similarly let $\Lambda \cong \mathbb{Z}^{\operatorname{rank}(G)}$ be the weight lattice of $\mathfrak{g}$ with dominant weights $\Lambda_{+}$. We fix an invariant bilinear form $(-,-)$on $\mathfrak{g}$, usually the Killing form. Let

$$
\delta=\frac{1}{2} \sum_{\alpha \in \triangle_{+}} \alpha
$$

be the Weyl vector of $\mathfrak{g}$. The vector $2 \delta$ is always a weight of $\mathfrak{g}$; if $G$ is semi-simple, then $\delta$ is also a weight and we can identify it with an integer vector $\delta \in \mathbb{Z}^{\operatorname{rank}(G)}$.

The partition function for the $q$-deformation of Yang-Mills theory with gauge group $G$ on a closed oriented Riemann surface $\Sigma_{h}$ of genus $h \geq 0$ can be written as a generalization 
of the Migdal heat kernel expansion given by [32]

$$
Z_{h, p}(q)=\sum_{\lambda \in \Lambda_{+}} \operatorname{dim}_{q}\left(R_{\lambda}\right)^{2-2 h} q^{\frac{p}{2}(\lambda+2 \delta, \lambda)},
$$

where $p \in \mathbb{Z}$ is a discrete parameter and the sum runs over all isomorphism classes of irreducible unitary representations $R_{\lambda}$ of $G$ which are parametrized by dominant weights $\lambda \in \Lambda_{+}$. The deformation parameter

$$
q=\mathrm{e}^{-g_{\mathrm{str}}}
$$

is identified with the coupling constant $g_{\text {str }}$ in topological string theory. The quantum dimension of the representation $R_{\lambda}$ labelled by $\lambda \in \Lambda_{+}$is

$$
\operatorname{dim}_{q}\left(R_{\lambda}\right)=\prod_{\alpha \in \triangle_{+}} \frac{[(\lambda+\delta, \alpha)]_{q}}{[(\delta, \alpha)]_{q}}
$$

where

$$
[x]_{q}=\frac{q^{x / 2}-q^{-x / 2}}{q-q^{-1}}
$$

for $x \in \mathbb{R}$ is a $q$-number. This theory is closely related to Chern-Simons theory on a principal U(1)-bundle of degree $p$ over $\Sigma_{h}$ [33].

For many computations it is useful to have an explicit expression for the partition function (2.10) in terms of highest weight variables. For this, we define shifted weights $\vec{k} \in \mathbb{Z}^{\operatorname{rank}(G)}$ by

$$
\vec{k}=\lambda+\delta,
$$

and use the Weyl reflection symmetry of the summand of the partition function (2.10) to remove the restriction to the fundamental chamber of the summation over $\vec{k}$. Up to overall normalization, the partition function (2.10) can thus be written as

$$
Z_{h, p}(q)=\sum_{\vec{k} \in\left(\mathbb{Z}^{\operatorname{rank}(G)}\right)_{\operatorname{reg}}} \Delta\left(g_{\text {str }} \vec{k}\right)^{2-2 h} \mathrm{e}^{-\frac{p g_{\text {str }}}{2}(\vec{k}, \vec{k})},
$$

where the Weyl determinant is given by

$$
\Delta(\vec{x})=\prod_{\alpha \in \triangle_{+}} 2 \sinh \frac{(\alpha, \vec{x})}{2}
$$

for $\vec{x}=\left(x_{1}, \ldots, x_{\operatorname{rank}(G)}\right) \in \mathbb{C}^{\operatorname{rank}(G)}$. The sum in (2.15) is restricted to those shifted weights where $\Delta\left(g_{\text {str }} \vec{k}\right)$ is non-zero, i.e. $(\alpha, \vec{k}) \neq 0$ for $\alpha \in \triangle_{+}$.

When the gauge group is the unitary group $G=\mathrm{U}(N)$, this two-dimensional gauge theory is conjecturally a non-perturbative completion of topological string theory on the local Calabi-Yau threefold which is the total space of the rank 2 holomorphic vector bundle

$$
\mathcal{O}_{\Sigma_{h}}(p+2 h-2) \oplus \mathcal{O}_{\Sigma_{h}}(-p) \longrightarrow \Sigma_{h}
$$


with $N$ D4-branes wrapping the exceptional divisor $\mathcal{O}_{\Sigma_{h}}(-p)$ and D2-branes wrapping the base $\Sigma_{h}$ [32]. In turn, for $h=0$ the two-dimensional theory defines an analytical continuation of Chern-Simons gauge theory on the lens space $L(p, 1)$ to the case where $q$ is not a root of unity [34]. In section 5 we shall find that five-dimensional cohomological localization over $S_{b}^{3} \times \Sigma_{h}$ gives a squashed deformation of this theory at $p=1$, which for genus $h=0$ and rational values $p / s$ of the squashing parameter $b^{2}$ is an analytical continuation of Chern-Simons theory on the more general lens spaces $L(p, s) \cong S_{b}^{3}$.

The correspondence with three-dimensional field theories has also been studied from other perspectives. In [35], the dimensional reduction of six-dimensional theories on $S^{1} \times$ $S_{b}^{3} \times \Sigma_{h}$ is considered by reducing to five dimensions as above, compactifying on $\Sigma_{h}$, and then obtaining a three-dimensional theory on the squashed sphere; this enables a comparison of the two possible compactification paths: first along $S^{1}$ and then on $\Sigma_{h}$ to obtain a theory on $S_{b}^{3}$, or first along $\Sigma_{h}$ to obtain a four-dimensional theory of class $\mathcal{S}$ and then relating it by standard reasoning to the three-dimensional partition function. In [36-38] the five-dimensional theory on $M_{3} \times S^{2}$ is obtained from dimensional reduction of the six-dimensional superconformal field theory on $S^{1} \times M_{3} \times S^{2}$ for more general threemanifolds $M_{3}$. The resulting theory on $M_{3}$ is related to complex Chern-Simons theory. In these cases the theory is partially twisted along $M_{3}$, and supersymmetric localization on $S^{2}$ is used to reduce to a twisted three-dimensional theory; this differs from the perspective of $[10,15]$, where the partial twist is along $\Sigma_{h}$ and localization over $S^{3}$ reduces the theory to two dimensions. In contrast, in this paper we will consider the fully twisted theory on $S_{b}^{3} \times \Sigma_{h}$. Finally, in [39], with squashed sphere of [29], the six-dimensional theory on $S_{b}^{3} \times M_{3}$ is reduced along the Hopf fibre of $S_{b}^{3}$, then twisted along $M_{3}$ and localized to reduce along $S^{2}$; the resulting three-dimensional theory is the same as in $[36,37]$.

\subsection{Basics of cohomological localization}

In this paper we use techniques based on localization theorems in equivariant cohomology, applied to supersymmetric quantum field theory, see e.g. [40, 41] for introductions to the subject; we will now briefly sketch the main ideas that will be used extensively in the remainder of this paper. Supersymmetric localization is a technique which allows the reduction of a supersymmetry preserving Euclidean path integral to an integral over the smaller set of fixed points of a supercharge Q. To compute the partition function, one adds a Q-exact term $\mathrm{Q} V$ to the action $S$ of the theory and computes the deformed partition function $Z(t)$ defined by functional integration of the Boltzmann weight $\mathrm{e}^{-S-t \mathrm{Q} V}$ for $t \in \mathbb{R}$; then $Z(0)$ is the original partition function we wish to compute. Supersymmetry of the path integral then implies that $Z(t)$ is formally independent of the parameter $t$, so that letting $t \rightarrow \infty$ and choosing the localizing term $V$ to be positive semi-definite, the functional integration reduces to a localization calculation around the fixed points $\mathrm{Q} V=0$ of the supercharge $\mathrm{Q}$. In general the set of fixed points is a superspace, with odd coordinates associated with supersymmetric fermionic modes that have vanishing action in the localizing term in the bosonic background.

In this paper we consider Euclidean manifolds preserving rigid supersymmetry with four and eight supercharges; we restrict them to non-trivial circle bundles $M_{2 n+1} \rightarrow K_{2 n}$, 
whose total spaces have odd dimension $2 n+1$, in order to avoid dealing with fermionic fixed points. We shall derive the fixed point loci based on cohomological forms of the (BRST) supersymmetry transformations which are compatible with the $\mathrm{U}(1)$-action on the circle bundle [11, 42]; this procedure is called topological twisting and the resulting theory is called a cohomological field theory (in the sense of equivariant cohomology). We shall also only consider localization on the Coulomb branch of the supersymmetric gauge theory, where the path integral is reduced to a finite-dimensional integral over a classical moduli space parameterized by scalars in vector multiplets, and holonomies and fluxes of gauge fields around non-contractible cycles in $M_{2 n+1}$ (possibly together with other continuous moduli).

The localization calculation amounts to computing a ratio of one-loop fluctuation determinants which is schematically given by [43]

$$
h(\phi)=\frac{\left.\operatorname{det} i \mathrm{~L}_{\phi}\right|_{\text {coker } \mathrm{D}}}{\left.\operatorname{det} \mathrm{i} \mathrm{L}_{\phi}\right|_{\operatorname{ker} \mathrm{D}}}
$$

where $\mathrm{D}$ denotes differential operators entering the localizing terms $V$, and $\mathrm{L}_{\phi}=-\mathrm{i} \mathrm{Q}^{2}$ generates the geometric $\mathrm{U}(1)$-action and gauge transformations parametrized by $\phi$ on the fields of the theory; the adjoint scalar $\phi$ is Q-closed and does not have a fermionic partner. Then the equivariant cohomology in the localization of the supersymmetric gauge theory consists of gauge-invariant states on the base space $K_{2 n}$ of the circle bundle, together with an infinite tower of Kaluza-Klein modes on the $S^{1}$ fibre. The effective contribution to this ratio from the zero modes which remain after cancellation between fermionic and bosonic states is computed using the Atiyah-Singer index theorem for transversally elliptic operators and the Atiyah-Bott localization formula in equivariant cohomology for the U(1)-action on $M_{2 n+1}$. The schematic form of the localized partition function is then given by

$$
Z\left(M_{2 n+1}\right)=\int_{\mathfrak{g}} \mathrm{d} \sigma \int_{\mathscr{M}_{G}^{\mathrm{BPS}}\left(M_{2 n+1}\right)} \mathrm{d} \boldsymbol{m} \mathrm{e}^{-S_{\mathrm{cl}}(\boldsymbol{m} ; \sigma)} Z_{\mathrm{vec}}\left(M_{2 n+1}\right) Z_{\mathrm{hyp}}\left(M_{2 n+1}\right),
$$

where $\mathfrak{g}$ is the Lie algebra of the gauge group $G$, and $\mathscr{M}_{G}^{\mathrm{BPS}}\left(M_{2 n+1}\right)$ is the BPS locus inside a moduli space of $G$-connections on $M_{2 n+1}$ parametrized by moduli $\boldsymbol{m}$. The action $S_{\mathrm{cl}}$ is the classical bosonic action, while $Z_{\text {vec }}$ and $Z_{\text {hyp }}$ are respectively the one-loop fluctuation determinants associated with the vector multiplet and the matter hypermultiplets of the supersymmetric gauge theory.

\subsection{Localization of $\mathcal{N}=1$ gauge theories on Seifert manifolds}

In this paper we mainly focus on Seifert manifolds which admit a free U(1) action, so that they admit a $\mathrm{U}(1)$ isometry. We shall comment where appropriate on the extension to more general principal U(1)-bundles over orbifolds, where the $\mathrm{U}(1)$ action has fixed points.

Geometric setup. Let $M_{2 n+1} \stackrel{\pi}{\longrightarrow} K_{2 n}$ be a circle bundle of degree $p$ over a compact Kähler manifold $\left(K_{2 n}, \omega\right)$ of real dimension $2 n$ with $[\omega] \in H^{2}\left(K_{2 n}, \mathbb{Z}\right)$. The almost contact structure $\kappa \neq 0$ on $M_{2 n+1}$ can be chosen to be a connection one-form on this bundle which is locally written as

$$
\kappa=\mathrm{d} \theta+p \pi^{*}(a)
$$


where $\theta \in[0,2 \pi r)$ is a local coordinate of the $S^{1}$ fibre and $a$ is a local symplectic potential for $\omega=\mathrm{d} a$. Then

$$
\mathrm{d} \kappa=p \pi^{*}(\omega)
$$

In contrast to $[12,13]$, we will not assume $\kappa$ to be a K-contact structure on $M_{2 n+1}$. Instead, our interest will mainly focus on product manifolds $M_{2 n+1}=M_{2 n-1} \times \Sigma_{h}$, where $M_{2 n-1}$ is a compact contact manifold, but in general not K-contact, and $\Sigma_{h}$ is a compact Riemann surface of genus $h$. Then $\omega=\omega_{K_{2 n-2}}+\omega_{\Sigma_{h}}$ is the sum of the symplectic forms on the base $K_{2 n-2}$ of the Seifert fibration of $M_{2 n-1}$ and $\Sigma_{h}$, and $\kappa \wedge(\mathrm{d} \kappa)^{\wedge(n-1)} \neq 0$ is proportional to the volume form on $M_{2 n-1}$ induced by a metric compatible with the contact structure. The canonical volume form on the total space $M_{2 n+1}$ is

$$
\mathrm{d} \Omega_{M_{2 n+1}}=\frac{(-1)^{n}}{2^{n-1}(n-1) !} \kappa \wedge(\mathrm{d} \kappa)^{\wedge(n-1)} \wedge \omega_{\Sigma_{h}} .
$$

The Reeb vector field $\xi$ is defined by the duality contraction

$$
\xi\llcorner\kappa=1
$$

and the invariance condition

$$
\mathcal{L}_{\xi} \kappa=\xi\llcorner\mathrm{d} \kappa=0,
$$

where $\mathcal{L}_{\xi}=\mathrm{d} \xi\llcorner+\xi\llcorner\mathrm{d}$ is the Lie derivative along $\xi$. It is the generator of the $\mathrm{U}(1)$-action on $M_{2 n+1}$, and in the coordinates (2.20) it assumes the form

$$
\xi=\frac{\partial}{\partial \theta}
$$

A natural choice of $\mathrm{U}(1)$-invariant metric on $M_{2 n+1}$ is given by

$$
\mathrm{d} s_{M_{2 n+1}}^{2}=\pi^{*}\left(\mathrm{~d} s_{K_{2 n}}^{2}\right)+\kappa \otimes \kappa
$$

where $\mathrm{d} s_{K_{2 n}}^{2}$ is the Kähler metric on $K_{2 n}$. Any $k$-form $\alpha$ on $M_{2 n+1}$ can be decomposed using the projector $\kappa \wedge \xi\llcorner$ into horizontal and vertical components as

$$
\alpha=\alpha_{H}+\alpha_{V}:=(1-\kappa \wedge \xi\llcorner) \alpha+\kappa \wedge \xi\llcorner\alpha,
$$

where $\xi\llcorner\alpha$ is the $k-1$-form component of $\alpha$ along the fibre direction.

The computation of the perturbative partition function of (twisted) $\mathcal{N}=1$ supersymmetric Yang-Mills theory on $M_{2 n+1}$ is described in [12,13], using equivariant localization techniques with respect to the $\mathrm{U}(1)$ action on $M_{2 n+1}$ and the maximal torus of the gauge group $G$. The relevant computations typically involve the determinant of the square of the (twisted) supercharge (equivariant differential), which is the kinetic operator

$$
\mathrm{L}_{\phi}=\mathcal{L}_{\xi}+\mathcal{G}_{\phi}
$$

acting on the tangent space to the space of fields, where $\mathcal{G}_{\phi}$ denotes the action by an element $\phi$ valued in the Cartan subalgebra of the Lie algebra $\mathfrak{g}$ of $G$; for fields in the vector 
multiplet of the supersymmetric gauge theory, $\mathcal{G}_{\phi}=\operatorname{ad}_{\phi}$ is the adjoint action. Here we assume momentarily that the localization locus consists of constant field configurations $\phi$; the case of non-constant $\phi$ is discussed below. The operator $\mathrm{L}_{\phi}$ acts with the same eigenvalue on both even and odd degrees in the spaces $\Omega_{H}^{(0, \bullet)}\left(M_{2 n+1}, \mathfrak{g}\right)$ of horizontal antiholomorphic $\mathfrak{g}$-valued forms. The cancellation between bosonic and fermionic fluctuation determinants in the localized path integral is determined by the index of the Dolbeault complex of $K_{2 n}$ twisted by the line bundles $\mathscr{L}^{\otimes m}$ for $m \in \mathbb{Z}$, where $\mathscr{L} \rightarrow K_{2 n}$ is the complex line bundle associated to the circle bundle $M_{2 n+1} \stackrel{\pi}{\longrightarrow} K_{2 n}$ with first Chern class $c_{1}(\mathscr{L})=p[\omega]$. Denoting the corresponding twisted Dolbeault operators as $\bar{\partial}^{(m)}$, the Atiyah-Singer index theorem gives the index as

$$
\text { index } \bar{\partial}^{(m)}=\int_{K_{2 n}} \operatorname{ch}\left(\mathscr{L}^{\otimes m}\right) \wedge \operatorname{Td}\left(T^{1,0} K_{2 n}\right),
$$

where $T^{1,0} K_{2 n}$ is the holomorphic tangent bundle of $K_{2 n}$, while ch and Td respectively denote the Chern character and the Todd class.

Geometry from rigid supersymmetry. We want to define a supersymmetric field theory on the backgrounds $M_{2 n+1}$ described above. In this paper we focus on theories with four supercharges, which means $\mathcal{N}=2$ theories in three dimensions and $\mathcal{N}=1$ theories in five dimensions; a thorough discussion on localization in five-dimensional superconformal field theories can be found in [44]. We follow the approach of [45]: we add a supergravity multiplet in flat spacetime, and then take the rigid limit of the supergravity theory. This is done in two steps: in the first step we set to zero all fermion fields, and in the second step we set to zero the supersymmetry variations of the fermion fields. The equation obtained by imposing the vanishing of the gravitino variation is called the (generalized) Killing spinor equation. The vector multiplet and hypermultiplets of the gauge theory are then coupled to the background values of the supergravity multiplet, and the theory is effectively put on a curved spacetime. For the description of supersymmetric backgrounds in three dimensions we mainly follow [46, 47], and in five dimensions we follow [48, 49].

Let $\varepsilon$ be a solution to the Killing spinor equation for $M_{2 n+1}$. We use it to define the vector field $v$ on $M_{2 n+1}$ as

$$
v^{\mu}=\varepsilon^{\dagger} \Gamma^{\mu} \varepsilon,
$$

where $\Gamma^{\mu}$ are the gamma-matrices in either three or five dimensions. The fact that $\varepsilon$ satisfies the Killing spinor equation guarantees that $v$ is a nowhere vanishing Killing vector field. In particular, its orbits foliate $M_{2 n+1}$.

As explained in [46, section 5] and in [47, section 4], in three dimensions there are two possibilities:

(I) The orbits of $v$ are closed. In this case $M_{3}$ is a Seifert manifold and $v$ coincides with the Reeb vector field $\xi$ of the $\mathrm{U}(1)$ fibration of $M_{3}$. Particular examples belonging to this class are the round sphere $S^{3}$ and the lens spaces $L(p, s)$.

(II) If the orbits of $v$ do not close, supersymmetry requires $M_{3}$ to have isometry group $\mathrm{U}(1) \times \mathrm{U}(1)$. In this case $M_{3}$ is a Seifert manifold but $v$ does not necessarily point 
along the U(1)-fibre. A particular example belonging to this class is the ellipsoid $S_{b}^{3}$ of $[28]$.

We will henceforth refer to the manifolds belonging to the setting (I) as regular, and to those of setting (II) as irregular. ${ }^{1}$ Irregular geometries may or may not admit a free U(1) action of the Reeb vector field; see the recent review [50] for a thorough description of the geometric approach to $\mathcal{N}=2$ supersymmetry on three-dimensional Seifert manifolds.

In the five-dimensional case, we will focus on product manifolds $M_{3} \times \Sigma_{h}$, where $\Sigma_{h}$ is a closed Riemann surface of genus $h$. Killing spinor solutions in these geometries are built from solutions on $M_{3}$, and thus an analogous discussion applies; see the discussion at the end of [49] for further details about the difference in the approach we follow here and that of $[13,51]$.

Examples: regular vs. irregular fibrations. We write down some explicit examples of regular and irregular five-dimensional manifolds. The round sphere $S^{5}$ and the product $S^{3} \times S^{2}$ are regular Sasaki-Einstein manifolds. The Sasaki-Einstein manifolds $Y^{p, s}$ studied in $[18,19]$ are irregular (or quasi-regular). The product manifolds $M_{3} \times \Sigma_{h}$ where $M_{3}$ is either $S^{3}, L(p, 1)$ or the three-dimensional torus $T^{3}$ are regular, while if $M_{3}=S_{b}^{3}$ is the ellipsoid of [28] it is irregular. Among the irregular manifolds, $S_{b}^{3} \times \Sigma_{h}$ (as well as replacing $S_{b}^{3}$ with other squashed Seifert three-manifolds) admits a free $\mathrm{U}(1)$ action, while $Y^{p, s}$ do not admit any free $\mathrm{U}(1)$ action and are described as U(1) fibrations over a warped product $S^{2} \rtimes S^{2}$. See appendix B for a classification and discussion of the different types of squashed three-spheres, and appendix $\mathrm{C}$ for a discussion about cohomological localization on $Y^{p, s}$.

One-loop determinants. For regular fibrations, it was shown in $[12,13]$ that the oneloop contribution of the $\mathcal{N}=1$ vector multiplet to the perturbative partition function on $M_{2 n+1}$ is

$$
Z_{\text {vec }}\left(M_{2 n+1}\right)=\prod_{\alpha \in \triangle}(\mathrm{i}(\alpha, \phi))^{d} \prod_{m \neq 0}\left(\frac{m}{r}+\mathrm{i}(\alpha, \phi)\right)^{\operatorname{index} \bar{\partial}^{(m)}},
$$

where as previously $\triangle$ is the root system of the Lie algebra $\mathfrak{g}$ and $(\cdot, \cdot)$ is an invariant non-degenerate bilinear form on $\mathfrak{g}$; the power $d$ of the first zero mode factor is given by

$$
d=\operatorname{index} \bar{\partial}-\operatorname{dim} H^{0}\left(M_{2 n+1}, \mathbb{R}\right),
$$

the difference between the index of the ordinary (untwisted) Dolbeault complex of $K_{2 n}$ and the dimension of the space of harmonic functions on $M_{2 n+1}$. The one-loop contribution from an $\mathcal{N}=1$ hypermultiplet in a representation $R$ of the gauge group $G$ is given by

$$
Z_{\mathrm{hyp}}\left(M_{2 n+1}\right)=\prod_{\rho \in \Lambda_{R}} \prod_{m \in \mathbb{Z}}\left(\frac{m}{r}+\mathrm{i}(\rho, \phi)+\frac{\Delta}{r}\right)^{-\operatorname{index} \bar{\partial}^{(m)}},
$$

\footnotetext{
${ }^{1}$ We will use the nomenclature "regular geometry" or "regular fibration", meaning that the integral curves of the Killing vector field have regular flow, and similarly for the "irregular fibration". In this paper we will not distinguish between irregular and quasi-regular cases.
} 
where $\Lambda_{R}$ is the lattice of weights of $R$ and $\Delta$ is a constant determined by the conformal scalar field coupling to the curvature in the gauge theory action. In [13] these formulas are applied to $\mathcal{N}=1$ gauge theory on the five-sphere $M_{5}=S^{5}$, viewed as a circle bundle over the projective plane $K_{4}=\mathbb{P}^{2}$, with $\Delta=\frac{3}{2}$.

The extension of these formulas to the case of non-constant scalar fields $\phi$ on $K_{2 n}$ can be deduced from the prescription explained in [52, appendix B], at least in the case when the kinetic operator $\mathrm{L}_{\phi}$ is elliptic. In these instances one can apply the index formula "locally" by moving the logarithms of the arguments of the products into the integral and integrating against the index density. For example, for the vector multiplet contribution this prescription gives

$$
\begin{aligned}
Z_{\text {vec }}\left(M_{2 n+1}\right)=\exp \left(\int_{K_{2 n}}\right. & \sum_{m \in \mathbb{Z}} \operatorname{ch}\left(\mathscr{L}^{\otimes m}\right) \wedge \operatorname{Td}\left(T^{1,0} K_{2 n}\right) \sum_{\alpha \in \triangle} \log \left(\frac{m}{r}+\mathrm{i}(\alpha, \phi)\right) \\
& \left.-\operatorname{dim} H^{0}\left(M_{2 n+1}, \mathbb{R}\right) \int_{K_{2 n}} \frac{\omega^{\wedge n}}{n !} \sum_{\alpha \in \triangle} \log (\mathrm{i}(\alpha, \phi))\right) .
\end{aligned}
$$

For the cohomological localization we shall employ in this paper, the further localization to constant $\phi$ in two dimensions will be immediate (in contrast to the approach of [10]).

The expressions $(2.31)$ and $(2.33)$ are proven in $[12,13]$ in the case of five-dimensional K-contact manifolds, with Killing vector field $v$ pointing along the Seifert fibre. In following sections we will review the main steps in the proof, both in three and five dimensions, and derive the corresponding expressions for the cases in which the Killing vector field $v$ does not point in the direction of the $\mathrm{U}(1)$ fibre. For this, we will have to introduce vector multiplets and hypermultiplets, and then topologically twist the field content. For the three-dimensional case we will follow the conventions of [11], while in five dimensions we follow $[13,53]$.

\section{$3 \mathcal{N}=2$ cohomological gauge theories in three dimensions}

In this section we study $\mathcal{N}=2$ supersymmetric gauge theories on three-dimensional manifolds. We will first present the theory and its topological twist. Then we will reproduce the formula for the one-loop determinants in the case of a Seifert fibration $M_{3} \rightarrow K_{2}$ corresponding to closed orbits of the Killing vector field $v$. We shall subsequently extend the formulas to the ellipsoid and ellipsoidal lens spaces, corresponding to non-compact orbits of the Killing vector field, by extending the application of the index theorem used in [14], for the ellipsoid, to any squashed Seifert manifold. For the geometric setting we will follow $[46,47]$, while for the topological twist and derivation of the one-loop determinants, as well as for the normalization of the fields and supersymmetry variations, we will continue to follow [11]. Our conventions are summarized in appendix A.

\subsection{Supersymmetric Yang-Mills theory and its cohomological formulation}

As usual, we start by placing the gauge theory on flat Euclidean space $\mathbb{R}^{3}$ and then couple it to background supergravity, following $[45,46]$. Let $\varepsilon$ and $\widetilde{\varepsilon}$ be two Killing spinors with 
opposite R-charge, and define the Killing vector field

$$
v^{\mu}=\widetilde{\varepsilon}^{\dagger} \gamma^{\mu} \varepsilon .
$$

Vector multiplet. The $\mathcal{N}=2$ vector multiplet in three dimensions consists of a gauge connection $A$, a real scalar $\sigma$, a complex spinor $\lambda$ (the gaugino), and an auxiliary real scalar $D$. The spinor $\lambda=\left(\lambda^{I}\right)$ and the real scalar $D=\left(D_{J}^{I}\right)$ carry $\mathrm{SU}(2)_{R}$ indices. $^{2}$ The supersymmetry transformations are standard $[11,54]$. We denote by $Q$ the equivariant differential (supersymmetry generator) which is the sum of the two independent supercharges $\frac{1}{2}\left(\widetilde{Q}_{\widetilde{\varepsilon}}+Q_{\varepsilon}^{\dagger}\right)$, and write

$$
\begin{aligned}
\mathrm{Q} A_{\mu} & =\frac{\mathrm{i}}{2}\left(\widetilde{\varepsilon}_{I}^{\dagger} \gamma_{\mu} \lambda^{I}-\lambda_{I}^{\dagger} \gamma_{\mu} \varepsilon^{I}\right), \\
\mathrm{Q} \sigma & =-\frac{1}{2}\left(\widetilde{\varepsilon}_{I}^{\dagger} \lambda^{I}+\lambda_{I}^{\dagger} \varepsilon^{I}\right), \\
\mathrm{Q} \lambda^{I} & =-\frac{1}{2} \gamma^{\mu \nu} \varepsilon^{I} F_{\mu \nu}-D^{I}{ }_{J} \varepsilon^{J}+\mathrm{i} \gamma^{\mu} \varepsilon^{I}\left(\mathrm{D}_{\mu} \sigma\right), \\
\mathrm{Q} \lambda_{I}^{\dagger} & =\frac{1}{2} \widetilde{\varepsilon}_{I}^{\dagger} \gamma^{\mu \nu} F_{\mu \nu}-\widetilde{\varepsilon}_{J}^{\dagger} D^{J}{ }_{I}-\mathrm{i} \gamma^{\mu} \widetilde{\varepsilon}_{I}^{\dagger}\left(\mathrm{D}_{\mu} \sigma\right), \\
\mathrm{Q} D_{I}{ }^{J} & =\frac{\mathrm{i}}{2}\left(\widetilde{\varepsilon}_{I}^{\dagger} \gamma^{\mu}\left(\mathrm{D}_{\mu} \lambda^{J}\right)-\left(\mathrm{D}_{\mu} \lambda_{I}^{\dagger}\right) \gamma^{\mu} \varepsilon^{J}\right)-\frac{\mathrm{i}}{2}\left(\widetilde{\varepsilon}_{I}^{\dagger}\left[\sigma, \lambda^{J}\right]-\left[\sigma, \lambda_{I}^{\dagger}\right] \varepsilon^{J}\right)+(I \leftrightarrow J),
\end{aligned}
$$

where $F$ is the curvature of the gauge connection $A$, and $\mathrm{D}_{\mu}$ is the covariant derivative which involves the gauge connection $A$ and also the spin connection when acting on the dynamical spinor fields $\lambda$ and $\lambda^{\dagger}$. When the theory is placed on a curved background $M_{3}$, one has to add curvature terms proportional to $\frac{1}{r}$ to the supersymmetry variations $Q \lambda$, $\mathrm{Q} \lambda^{\dagger}$ and $\mathrm{Q} D$. These terms will also involve the spinor covariant derivative acting on the Killing spinors from the supergravity background. This procedure is standard and we do not review it here.

Following [11, appendix A], we use the Killing vector field $v$ for the topological twist. We set $\widetilde{\varepsilon}=\varepsilon$, and rewrite the spinor fields $\lambda$ and $\lambda^{\dagger}$ in the vector multiplet in terms of an odd $\mathfrak{g}$-valued one-form $\Psi$ and an odd $\mathfrak{g}$-valued zero-form $\chi$ defined as

$$
\Psi_{\mu}=\frac{1}{2}\left(\varepsilon^{\dagger} \gamma_{\mu} \lambda-\lambda^{\dagger} \gamma_{\mu} \varepsilon\right) \quad \text { and } \quad \chi=\varepsilon^{\dagger} \lambda-\lambda^{\dagger} \varepsilon,
$$

which depend on the solution $\varepsilon$ of the Killing spinor equation, and hence on the choice of contact structure, but not explicitly on the metric. The field content of the vector multiplet is now written in a cohomological form as

$$
A \in \Omega^{1}\left(M_{3}, \mathfrak{g}\right), \quad \sigma \in \Omega^{0}\left(M_{3}, \mathfrak{g}\right), \quad \Psi \in \Omega^{1}\left(M_{3}, \mathfrak{g}\right) \quad \text { and } \quad \chi \in \Omega^{0}\left(M_{3}, \mathfrak{g}\right),
$$

with $(A, \chi)$ treated as coordinates and $(\sigma, \Psi)$ as conjugate momenta on field space. We do not include details about the gauge fixing here, and again refer to [11] for the technical details. It suffices to say that the bosonic ghost coordinates are a pair of harmonic zero-forms

\footnotetext{
${ }^{2}$ By analogy with the five-dimensional setting, we work with $\mathrm{SU}(2)_{R}$ R-symmetry. For a generic $\mathcal{N}=2$ theory with only $\mathrm{U}(1)_{R}$ R-symmetry, simply neglect the indices $I, J$.
} 
and the fermionic ghost coordinates are a pair of zero-forms. We use the localizing term

$$
\mathrm{Q} V \quad \text { with } \quad V=\int_{M_{3}}\left((\mathrm{Q} \lambda)^{\dagger} \lambda+\lambda^{\dagger}\left(\mathrm{Q} \lambda^{\dagger}\right)^{\dagger}\right) \mathrm{d} \Omega_{M_{3}}
$$

in the path integral which brings the quantum field theory to the fixed point locus

$$
F=0 \quad \text { and } \quad \sigma=-D=\text { constant } .
$$

The kinetic operator. Once the fields are in cohomological form, the supersymmetry transformation squares to

$$
\mathrm{Q}^{2}=\mathrm{iL}_{\phi} \quad \text { with } \quad \mathrm{L}_{\phi}=\mathcal{L}_{v}+\mathcal{G}_{\phi} .
$$

Here $\mathrm{L}_{\phi}$ is the sum of a Lie derivative along $v$ and a gauge transformation $\mathcal{G}_{\phi}$ with parameter

$$
\phi=\mathrm{i} \sigma-v\llcorner A .
$$

At the end, we shall rotate $\sigma \mapsto \mathrm{i} \sigma_{0}$ and integrate over real $\sigma_{0} \in \mathfrak{g}$. The localization locus consists of flat connections, and therefore

$$
\phi=-\left(\sigma_{0}+v^{\mu} A_{\mu}^{(0)}\right) \in \mathfrak{g},
$$

where $A^{(0)}$ is the point of the moduli space of flat $G$-connections on $M_{3}$ around which we are expanding. If $M_{3}$ is simply connected, the only point of the moduli space is the trivial connection. Otherwise, expanding around $A^{(0)}=0$ gives the perturbative part of the partition function. In general, the full answer is given by integrating the partition function over the moduli space of flat $G$-connections $\mathscr{M}_{G}^{0}\left(M_{3}\right)$ supported on $M_{3}$, which is given by

$$
\mathscr{M}_{G}^{0}\left(M_{3}\right)=\operatorname{Hom}\left(\pi_{1}\left(M_{3}\right), G\right) / G,
$$

where the quotient is taken by the conjugation action of $G$ on the holonomy of a connection over representatives of elements in $\pi_{1}\left(M_{3}\right)$. When $M_{3}$ is a circle bundle of degree $p$ over a compact oriented Riemann surface $C_{g}$ of genus $g$, there is an explicit presentation of the fundamental group $\pi_{1}\left(M_{3}\right)$ with generators $a_{i}, b_{i}, \zeta, i=1, \ldots, g$ and the relation

$$
\prod_{i=1}^{g}\left[a_{i}, b_{i}\right]=\zeta^{p},
$$

with all other pairwise combinations of generators commuting. An explicit parametrization of the moduli space (3.10) in the case $G=\mathrm{U}(N)$ can be found in [55, section 6.2].

Hypermultiplets. The field content of an $\mathcal{N}=2$ hypermultiplet in three dimensions consists of the complex scalars $\mathrm{q}=\left(\mathrm{q}_{I}\right)$ with $\mathrm{SU}(2)_{R}$ indices and a complex spinor $\psi$. These fields are obtained by combining chiral and anti-chiral complex scalars and Weyl spinors. One also needs an auxiliary complex scalar. The supersymmetry transformations are

$$
\begin{aligned}
\mathrm{Qq}_{I} & =-\mathrm{i} \widetilde{\varepsilon}_{I}^{\dagger} \psi, \\
\mathrm{Q} \psi & =\frac{1}{2} \gamma^{\mu} \varepsilon^{I}\left(\mathrm{D}_{\mu} \mathrm{q}_{I}\right)+\frac{\mathrm{i}}{2} \sigma \mathrm{q}_{I} \varepsilon^{I},
\end{aligned}
$$


plus curvature corrections to be added when the theory is put on $M_{3}$. The transformations of the conjugate fields $\mathrm{q}^{\dagger}, \psi^{\dagger}$ are the obvious ones, with exchange $\varepsilon \leftrightarrow \widetilde{\varepsilon}$. The topological twist of a hypermultiplet was first performed in [42, appendix B].

The Killing spinors are used to introduce a new spinor field

$$
\mathrm{q}^{\prime}=\mathrm{q}_{I} \varepsilon^{I},
$$

so that the physical fields are all reformulated in terms of spinors. These fields are singlets under the action of $\mathrm{SU}(2)_{R}$; this is instrumental to have the kinetic operator in the desired form. One finds

$$
\mathrm{Q}^{2}=\mathrm{i} \mathrm{L}_{\phi} \quad \text { with } \quad \mathrm{L}_{\phi}=\mathcal{L}_{v}^{\text {spin }}+\mathcal{G}_{\phi},
$$

as in the vector multiplet. We used the notation $\mathcal{L}_{v}^{\text {spin }}$ to stress that the Lie derivative is twisted by the spin covariant derivative when acting on spinors on curved manifolds.

To mimic the procedure of [11], a further step is needed: we rearrange the fields again in a cohomological form, combining spinors into differential forms. For this, we need to define a spin ${ }^{c}$ structure on $M_{3}$, and use it to decompose the spinors $\psi_{ \pm}$and $\mathrm{q}^{\prime}$ into elements of

$$
\Omega_{H}^{0}\left(M_{3}, \mathfrak{g}\right) \oplus \Omega_{H}^{(0,1)}\left(M_{3}, \mathfrak{g}\right)
$$

where we decomposed $\psi$ according to the chirality operator as

$$
\psi=\psi_{+}+\psi_{-} \quad \text { with } \quad \gamma_{5} \psi_{ \pm}= \pm \psi_{ \pm} \quad \text { and } \quad \gamma_{5}=v^{\mu} \gamma_{\mu} .
$$

The $\mathrm{SU}(2)_{R}$ R-symmetry is not considered in [42], and they do not pass through the intermediate step of contracting the $\mathrm{SU}(2)_{R}$ indices to form singlets; instead, they directly define anti-holomorphic forms. In the present case, the cohomological field theory contains hypermultiplets only when the manifold $M_{3}$ admits a $\operatorname{spin}^{c}$ structure.

\subsection{One-loop determinant of the vector multiplet in a regular background}

Gaussian integration of the vector multiplet around the fixed point $\phi$ gives the ratio of fluctuation determinants

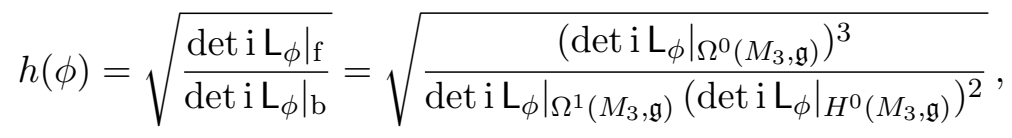

where the subscripts on the left-hand side refer to the operator acting on fermionic or bosonic fields. Here $H^{0}\left(M_{3}, \mathfrak{g}\right)$ is the space of $\mathfrak{g}$-valued harmonic zero-forms, and $\mathbf{L}_{\phi}$ is given in (3.7). The numerator of $h(\phi)$ includes the contributions from the fermionic field $\chi$ and the two fermionic ghost fields, while the denominator includes the contributions from the bosonic field $A$ and the two bosonic ghost fields.

We use the Seifert structure of $M_{3}$ to decompose one-forms into horizontal and vertical parts as

$$
\Omega^{1}\left(M_{3}, \mathfrak{g}\right)=\Omega_{V}^{1}\left(M_{3}, \mathfrak{g}\right) \oplus \Omega_{H}^{1}\left(M_{3}, \mathfrak{g}\right) \cong \Omega^{0}\left(M_{3}, \mathfrak{g}\right) \oplus \Omega_{H}^{1}\left(M_{3}, \mathfrak{g}\right) .
$$

We may also identify

$$
\Omega_{H}^{2}\left(M_{3}, \mathfrak{g}\right) \cong \Omega^{0}\left(M_{3}, \mathfrak{g}\right)
$$


in three dimensions. The circle bundle structure

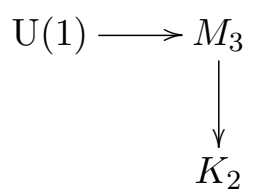

allows us to further decompose the spaces of zero-forms and horizontal one-forms as

$$
\begin{aligned}
& \Omega^{0}\left(M_{3}, \mathfrak{g}\right)=\Omega^{0}\left(K_{2}, \mathfrak{g}\right) \oplus \bigoplus_{m \neq 0} \Omega^{0}\left(K_{2}, \mathscr{L}^{\otimes m} \otimes \mathfrak{g}\right), \\
& \Omega_{H}^{1}\left(M_{3}, \mathfrak{g}\right)=\Omega^{1}\left(K_{2}, \mathfrak{g}\right) \oplus \bigoplus_{m \neq 0} \Omega^{1}\left(K_{2}, \mathscr{L}^{\otimes m} \otimes \mathfrak{g}\right),
\end{aligned}
$$

where we recall that $\mathscr{L}$ is the line bundle associated to the $\mathrm{U}(1)$ fibration of $M_{3}$. Using the short-hand notation

$$
\left.\mathcal{D}_{m}^{\bullet}(\phi)=\left.\operatorname{det} \mathrm{iL}_{\phi}\right|_{\Omega \bullet\left(K_{2}, \mathscr{L} \otimes m\right.} \otimes \mathfrak{g}\right)
$$

we obtain



The crucial observation at this point is that when the Killing vector field $v$ points along the fibre direction, the decomposition (3.21) corresponds to a decomposition in eigenmodes of the Lie derivative operator $\mathcal{L}_{v}$. The degeneracy of the action of the gauge transformations $\mathcal{G}_{\phi}$ is resolved in the standard way $[11,54]$, by decomposing the Lie algebra $\mathfrak{g}$ into its root system as

$$
\mathfrak{g}=\bigoplus_{\alpha \in \triangle} \mathfrak{g}_{\alpha}
$$

We finally obtain

$$
Z_{\mathrm{vec}}\left(M_{3}\right)=\prod_{\alpha \in \triangle}(\mathrm{i}(\alpha, \phi))^{\frac{1}{2} \chi\left(K_{2}\right)-\operatorname{dim} H^{0}\left(M_{3}, \mathbb{R}\right)} \prod_{m \neq 0}\left(\frac{m}{r}+\mathrm{i}(\alpha, \phi)\right)^{\operatorname{index} \bar{\partial}^{(m)}}
$$

where we used the fact that the number of remaining modes, after cancellation, is given by the index of the twisted Dolbeault complex

$$
\Omega^{0}\left(K_{2}, \mathscr{L}^{\otimes m}\right) \stackrel{\bar{\partial}^{(m)}}{\longrightarrow} \Omega^{1}\left(K_{2}, \mathscr{L}^{\otimes m}\right) \stackrel{\bar{\partial}^{(m)}}{\longrightarrow} \Omega^{2}\left(K_{2}, \mathscr{L}^{\otimes m}\right),
$$

after identification of the complexified de Rham differential with the anti-holomorphic Dolbeault differential. This result agrees with [56]. The first multiplicative term in (3.25) is trivial for Seifert homology spheres. The proof of this cohomological localization formula used the properties that $M_{3}$ is a Seifert manifold and that $v$ is parallel to the Reeb vector field $\xi$, and hence that $M_{3}$ is a K-contact manifold. 


\subsection{One-loop determinant of the vector multiplet in an irregular background}

We now consider the case in which the orbits of the Killing vector field $v$ are not closed. The background geometry is required to have $\mathrm{U}(1) \times \mathrm{U}(1)$ isometry group in order to preserve supersymmetry [46, 47]. In this instance $M_{3}$ is still a Seifert manifold but now $v$ does not point along the fibre. In the spirit of section 3.2, we calculate the ratio of fluctuation determinants through the index theorem. A similar calculation was performed in [14], but there the supersymmetry transformation squares to the sum of a Lie derivative along $v$, a gauge transformation and a third transformation which is a sum of R-symmetry and flavour symmetry transformations (determining a new R-symmetry); this is not of the form described in [11, 12], and so our index theory calculations cannot be applied directly in this framework.

We can express $v$ as a linear combination

$$
v=a_{1} \xi+a_{2} \widetilde{\xi}
$$

of the Reeb vector field $\xi$ and a vector field $\widetilde{\xi}$ which generates a residual U(1) isometry. They are mutually orthogonal and are linear combinations of the generators of the torus isometry. Most of the calculation follows that of the round case from section 3.2, particularly the decomposition (3.21) of $\Omega^{\bullet}\left(M_{3}, \mathfrak{g}\right)$ according to the Seifert fibration. However, we now have to face the problem that the eigenmodes of $\mathcal{L}_{\xi}$ are no longer eigenmodes of $\mathcal{L}_{v}$.

In [14] it was shown how one can exploit the fact that if the $\mathrm{U}(1)$ action generated by the Reeb vector field $\xi$ is free, then the problem can be reduced to the quotient space $M_{3} / \mathrm{U}(1) \cong K_{2}$. Let us elaborate a bit more on this point. The crucial observation is that the restriction of the operator $i L_{\phi}$ is no longer elliptic, but it is transversally elliptic with respect to the isometry generated by $\widetilde{\xi}$. Following [57], given a first order transversally elliptic differential operator and a subgroup which acts freely, the index can be computed on the quotient space and the Atiyah-Bott localization formula localizes the contributions to the fixed points of the action of the subgroup generated by $\widetilde{\xi}$; this works even when $K_{2}$ possesses orbifold points. We then decompose into eigenmodes corresponding to $\widetilde{\xi}$, and the remaining modes after cancellation come from the fixed points of the $\mathrm{U}(1)$ action generated by $\widetilde{\xi}$. Therefore for irregular Seifert manifolds we obtain

$$
Z_{\mathrm{vec}}\left(M_{3}\right)=\prod_{\alpha \in \triangle}(\mathrm{i}(\alpha, \phi))^{\frac{1}{2} \chi\left(K_{2}\right)-\operatorname{dim} H^{0}\left(M_{3}, \mathbb{R}\right)} \prod_{f} \prod_{m \neq 0}\left(\frac{m}{\epsilon_{f}}+\mathrm{i}(\alpha, \phi)\right)^{\frac{1}{2} \mathrm{index} \bar{\partial}^{(m)}}
$$

where the second product runs over the fixed points of the $\mathrm{U}(1)$ action generated by $\widetilde{\xi}$ on $K_{2}$, and $\epsilon_{f}$ is the radius of the circle fibre over the fixed point labelled by $f .{ }^{3}$ This formula gives the correct answer for ellipsoids [28, 47], and generalizes the result of [14] to any Seifert manifold which is not K-contact. Notice that an ellipsoid Seifert manifold is necessarily fibered topologically over a sphere $S^{2}$ with at most two punctures [50, section 3.5].

\subsection{One-loop determinant of a hypermultiplet}

For the Gaussian integration of a hypermultiplet around a fixed point $\phi$, we do not give all the details here since the computation is essentially the same as for the vector multiplet.

\footnotetext{
${ }^{3}$ The square roots of each fixed point contribution come from the square root of the original ratio of fluctuation determinants. In the regular case, the contributions are equal.
} 
One finds

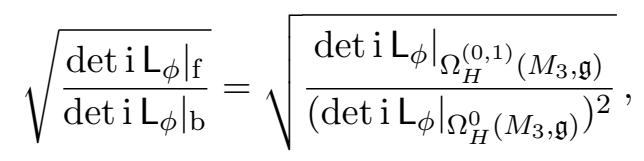

where we used the topological twist described in section 3.1. Recall that this formula only holds if we are allowed to recombine the $\mathrm{SU}(2)_{R}$ singlet spinors $\mathrm{q}^{\prime}$ into anti-holomorphic differential forms. This fails for the ellipsoid $S_{b}^{3}$ of [28], for instance, because a $\operatorname{spin}^{c}$ structure is not guaranteed to exist when the dual one-form to the Killing vector $v$ is not a gauge connection for the $\mathrm{U}(1)$ fibration.

For round Seifert manifolds, the eigenvalues of $\mathcal{L}_{v}^{\text {spin }}$ are

$$
-\frac{\mathrm{i} m}{r}-\frac{\mathrm{i} \Delta}{r}
$$

where $m \in \mathbb{Z}$ and $\Delta$ is the R-charge the hypermultiplet, which we assume equal to the conformal dimension, although more general assignments are possible in theories with only four supercharges. The number of remaining modes after cancellations in (3.29) is given by the index of the twisted Dolbeault differential, and we obtain

$$
Z_{\text {hyp }}\left(M_{3}\right)=\prod_{\rho \in \Lambda_{R}} \prod_{m \in \mathbb{Z}}\left(\frac{m+\Delta}{r}+\mathrm{i}(\rho, \phi)\right)^{-\operatorname{index} \bar{\partial}^{(m)}}
$$

where a shift $m \rightarrow m+\Delta$ should be included when considering twisted boundary conditions along the fibre, but the contribution of this shift cancels in the computations. This result agrees with [56].

\subsection{Applications of the cohomological localization formulas}

We shall now provide some simple examples illustrating how the localization formulas obtained in this section work to give the correct known results in three dimensions.

Localization on $\boldsymbol{S}^{\mathbf{3}}$. As a first check, let us examine how to reproduce the threedimensional localization calculations of [54, section 3] in this framework. We consider the three-sphere $M_{3}=S^{3}$ of radius $r$, viewed as a circle bundle of degree one over the projective line $K_{2}=\mathbb{P}^{1}$ via the Hopf fibration, with Euler characteristic $\chi\left(\mathbb{P}^{1}\right)=2$ and $H^{0}\left(S^{3}, \mathbb{R}\right)=\mathbb{R}$. The total Chern class of the holomorphic tangent bundle of $\mathbb{P}^{1}$ is

$$
c\left(T^{1,0} \mathbb{P}^{1}\right)=(1+\omega)^{\wedge 2}=1+2 \omega=1+c_{1}\left(T^{1,0} \mathbb{P}^{1}\right)
$$

and so the corresponding Todd class is given by

$$
\operatorname{Td}\left(T^{1,0} \mathbb{P}^{1}\right)=1+\frac{1}{2} c_{1}\left(T^{1,0} \mathbb{P}^{1}\right)=1+\omega
$$

while the Chern characters of the line bundles $\mathscr{L}^{\otimes m} \rightarrow \mathbb{P}^{1}$ are given by

$$
\operatorname{ch}\left(\mathscr{L}^{\otimes m}\right)=1+c_{1}\left(\mathscr{L}^{\otimes m}\right)=1+m c_{1}(\mathscr{L})=1+m \omega
$$


The index of the corresponding twisted Dolbeault complex is thus given by

$$
\operatorname{index} \bar{\partial}^{(m)}=\int_{\mathbb{P}^{1}}(1+m \omega) \wedge(1+\omega)=\int_{\mathbb{P}^{1}}(m+1) \omega=m+1 .
$$

The one-loop vector multiplet contribution is then computed to be

$$
\begin{aligned}
Z_{\mathrm{vec}}\left(S^{3}\right) & =\prod_{\alpha \in \triangle} \prod_{m \neq 0}\left(\frac{m}{r}+\mathrm{i}(\alpha, \phi)\right)^{m+1} \\
& =\prod_{\alpha \in \triangle} \prod_{m=1}^{\infty} \frac{\left(\frac{m}{r}-\mathrm{i}\left(\alpha, \sigma_{0}\right)\right)^{m+1}}{\left(-\frac{m}{r}-\mathrm{i}\left(\alpha, \sigma_{0}\right)\right)^{m-1}} \\
& =\prod_{\alpha \in \triangle_{+}} \prod_{m=1}^{\infty} \frac{\left(\frac{m^{2}}{r^{2}}+\left(\alpha, \sigma_{0}\right)^{2}\right)^{m+1}}{\left(\frac{m^{2}}{r^{2}}+\left(\alpha, \sigma_{0}\right)^{2}\right)^{m-1}} \\
& =\prod_{\alpha \in \triangle_{+}} \prod_{m=1}^{\infty} \frac{m^{4}}{r^{4}}\left(1+\frac{r^{2}\left(\alpha, \sigma_{0}\right)^{2}}{m^{2}}\right)^{2} \\
& =\prod_{\alpha \in \triangle_{+}}\left(\frac{2 \sinh \pi r\left(\alpha, \sigma_{0}\right)}{\pi r\left(\alpha, \sigma_{0}\right)}\right)^{2},
\end{aligned}
$$

where as previously $\triangle_{+}$is the system of positive roots of the Lie algebra $\mathfrak{g}$, and we used the fact that the roots come in positive-negative pairs. In the second line we used the fact that the only flat connection on $S^{3}$ is trivial and substituted $\phi=-\sigma_{0}$, and in the last line we evaluated the infinite product using zeta-function regularization:

$$
\prod_{m=1}^{\infty}\left(1+\frac{x^{2}}{m^{2}}\right)=\frac{\sinh (\pi x)}{\pi x} \quad \text { and } \quad \prod_{m=1}^{\infty} \frac{m^{2}}{r^{2}}=2 \pi r .
$$

The same calculation for a one-loop hypermultiplet determinant gives

$$
\begin{aligned}
Z_{\text {hyp }}\left(S^{3}\right) & =\prod_{\rho \in \Lambda_{R}} \prod_{m \in \mathbb{Z}}\left(\frac{m+\Delta}{r}+\mathrm{i}(\rho, \phi)\right)^{-1-m} \\
& =\prod_{\rho \in \Lambda_{R}} \frac{\prod_{m=0}^{\infty}\left(\frac{-m-1+\Delta}{r}-\mathrm{i}\left(\rho, \sigma_{0}\right)\right)^{m}}{\prod_{m=1}^{\infty}\left(\frac{m-1+\Delta}{r}+\mathrm{i}\left(\rho, \sigma_{0}\right)\right)^{m}} \\
& =\prod_{\rho \in \Lambda_{R}} \prod_{m=1}^{\infty}\left(\frac{\frac{m+1-\Delta}{r}+\mathrm{i}\left(\rho, \sigma_{0}\right)}{\frac{m-1+\Delta}{r}-\mathrm{i}\left(\rho, \sigma_{0}\right)}\right)^{m} \\
& =\prod_{\rho \in \Lambda_{R}} \mathrm{~s}_{1}\left(\mathrm{i}(1-\Delta)-r\left(\rho, \sigma_{0}\right)\right)
\end{aligned}
$$

where in the last line we inserted the definition of the double-sine function which is the meromorphic function defined by the zeta-function regularized infinite products [58]

$$
\mathbf{s}_{b}(x)=\prod_{m, n=0}^{\infty} \frac{m b+n b^{-1}+\frac{1}{2}\left(b+b^{-1}\right)-\mathrm{i} x}{m b+n b^{-1}+\frac{1}{2}\left(b+b^{-1}\right)+\mathrm{i} x}
$$

evaluated at $b=1$. These results all agree with the computations of [54, section 3.2] (see also $[14$, section 3.2]). 
Localization on ellipsoid $S_{b}^{3}$ and $L(p, 1)_{b}$. We now consider the ellipsoid $S_{b}^{3}$ of [28], with squashing parameter $b>0$ and metric

$$
\mathrm{d} s_{S_{b}^{3}}^{2}=r^{2}\left(f(\vartheta)^{2} \mathrm{~d} \vartheta \otimes \mathrm{d} \vartheta+b^{2} \cos ^{2} \vartheta \mathrm{d} \varphi_{1} \otimes \mathrm{d} \varphi_{1}+b^{-2} \sin ^{2} \vartheta \mathrm{d} \varphi_{2} \otimes \mathrm{d} \varphi_{2}\right)
$$

induced from the standard metric on $\mathbb{C}^{2}$ restricted to the locus

$$
b^{2}\left|z_{1}\right|^{2}+b^{-2}\left|z_{2}\right|^{2}=r^{2}
$$

where $f(\vartheta)=\sqrt{b^{2} \cos ^{2} \vartheta+b^{-2} \sin ^{2} \vartheta}$ and $\left(\vartheta, \varphi_{1}, \varphi_{2}\right)$ are Hopf coordinates on the usual round sphere $S^{3}=S_{b=1}^{3}$ of radius $r$. This defines an irregular fibration with isometry group $\mathrm{U}(1) \times \mathrm{U}(1)$ : the Killing vector field $v$ takes the form

$$
v=\frac{b+b^{-1}}{2 r} \xi+\frac{b-b^{-1}}{2 r} \widetilde{\xi},
$$

where $\xi=\frac{\partial}{\partial \theta}$ is the Reeb vector field of the Seifert fibration $S_{b}^{3} \rightarrow \mathbb{P}^{1}$, with $\theta=\frac{1}{2}\left(\varphi_{1}+\varphi_{2}\right)$, and $\widetilde{\xi}=\frac{\partial}{\partial \widetilde{\theta}}$ is the generator of the residual $\mathrm{U}(1)$ isometry, with $\widetilde{\theta}=\frac{1}{2}\left(\varphi_{1}-\varphi_{2}\right)$. The fixed points of $\widetilde{\xi}$ correspond to the north and south poles of the base $S^{2} \cong \mathbb{P}^{1}$, with respective coordinates $\vartheta=0$, at which the fibre has radius $\epsilon_{1}=r b$, and $\vartheta=\frac{\pi}{2}$, at which the fibre has radius $\epsilon_{2}=r b^{-1}$. The index of the twisted Dolbeault complex is a topological invariant, and hence is the same as for round $S^{3}$.

Altogether, the localization formula gives

$$
\begin{aligned}
Z_{\mathrm{vec}}\left(S_{b}^{3}\right) & =\prod_{\alpha \in \triangle} \prod_{m \neq 0}\left(\left(\frac{m b^{-1}}{r}-\mathrm{i}\left(\alpha, \sigma_{0}\right)\right)^{\frac{1}{2}}\left(\frac{m b}{r}-\mathrm{i}\left(\alpha, \sigma_{0}\right)\right)^{\frac{1}{2}}\right)^{m+1} \\
& =\prod_{\alpha \in \triangle} \prod_{m=1}^{\infty} \frac{\left(\frac{m b^{-1}}{r}-\mathrm{i}\left(\alpha, \sigma_{0}\right)\right)^{\frac{m+1}{2}}\left(\frac{m b}{r}-\mathrm{i}\left(\alpha, \sigma_{0}\right)\right)^{\frac{m+1}{2}}}{\left(\frac{m b^{-1}}{r}+\mathrm{i}\left(\alpha, \sigma_{0}\right)\right)^{\frac{m-1}{2}}\left(\frac{m b}{r}+\mathrm{i}\left(\alpha, \sigma_{0}\right)\right)^{\frac{m-1}{2}}} \\
& =\prod_{\alpha \in \triangle_{+}} \prod_{m=1}^{\infty}\left(\frac{m^{2} b^{-2}}{r^{2}}+\left(\alpha, \sigma_{0}\right)^{2}\right)\left(\frac{m^{2} b^{2}}{r^{2}}+\left(\alpha, \sigma_{0}\right)^{2}\right) \\
& =\prod_{\alpha \in \triangle_{+}} \frac{\sinh \left(\pi b r\left(\alpha, \sigma_{0}\right)\right) \sinh \left(\pi b^{-1} r\left(\alpha, \sigma_{0}\right)\right)}{\pi^{2} r^{2}\left(\alpha, \sigma_{0}\right)^{2}}
\end{aligned}
$$

which agrees with [28, eq. (5.33)] and [14, eq. (4.24)]. This result is independent of the particular form of the smooth squashing function $f(\vartheta)$ and depends only on its values at the fixed points $\vartheta=0, \frac{\pi}{2}$; it can therefore be extended to a larger class of backgrounds with the same topology [59].

This result straightforwardly extends to the ellipsoid lens spaces $L(p, 1)_{b}$, with the induced metric on the quotient $S_{b}^{3} / \mathbb{Z}_{p}$ and associated line bundle $\mathscr{L} \rightarrow \mathbb{P}^{1}$ of degree $p$, so that now $c_{1}(\mathscr{L})=p \omega$; see the review [60] for a description of localization on $L(p, 1)_{b}$. The modifications are the same as for the round case, and amount to a shift $\sigma_{0} \mapsto \sigma_{0}+v^{\mu} A_{\mu}^{(0)}$, where $A^{(0)}$ is an isolated point of the moduli space of flat $G$-connections on $L(p, 1)$. Such flat connections are classified by conjugacy classes of embeddings of the fundamental group 
$\pi_{1}\left(L(p, 1)_{b}\right)=\mathbb{Z}_{p}$ in the gauge group $G$, and are in one-to-one correspondence with arrays $\vec{m} \in\left(\mathbb{Z}_{p}\right)^{\operatorname{rank}(G)}$ modulo Weyl symmetry. For the vector multiplet, the localization formula then gives

$$
Z_{\mathrm{vec}}\left(L(p, 1)_{b}\right)=\prod_{\alpha \in \triangle_{+}} \frac{\sinh \left(\frac{\pi b r}{p}\left(\alpha, \sigma_{0}+\mathrm{i} \vec{m}\right)\right) \sinh \left(\frac{\pi b^{-1} r}{p}\left(\alpha, \sigma_{0}+\mathrm{i} \vec{m}\right)\right)}{\frac{\pi^{2} r^{2}}{p^{2}}\left(\alpha, \sigma_{0}+\mathrm{i} \vec{m}\right)^{2}} .
$$

With similar modifications, one can extend these calculations to any ellipsoid Seifert manifold.

There is no $\operatorname{spin}^{c}$ structure on $S_{b}^{3}$ that can be used to apply our index theory formalism to the hypermultiplet contributions. Nevertheless, the one-loop fluctuation determinant can still be calculated in this case. For example, one could split the twisted Lie derivative as

$$
\mathcal{L}_{v}^{\text {spin }}=\frac{2 b}{r} \mathcal{L}_{\frac{\partial}{\partial \varphi_{1}}}^{\text {spin }}+\frac{2 b^{-1}}{r} \mathcal{L}_{\frac{\partial}{\partial \varphi_{2}}}^{\text {spin }}
$$

and decompose the fields into eigenmodes of the Lie derivatives in the two orthogonal toroidal directions $\frac{\partial}{\partial \varphi_{1}}$ and $\frac{\partial}{\partial \varphi_{2}}$, whose corresponding eigenvalues are then of the form $\frac{m+\Delta}{\epsilon_{1}}+\frac{n+\Delta}{\epsilon_{2}}$ where $m, n \in \mathbb{Z}$. Then the one-loop contribution of a hypermultiplet in a representation $R$ of the gauge group $G$ is given by [14, section 4 ]

$$
\begin{aligned}
Z_{\text {hyp }}\left(S_{b}^{3}\right) & =\prod_{\rho \in \Lambda_{R}} \prod_{m, n=0}^{\infty} \frac{m b+n b^{-1}+\left(b+b^{-1}\right)\left(1-\frac{\Delta}{2}\right)+\mathrm{i} r\left(\rho, \sigma_{0}\right)}{m b+n b^{-1}+\left(b+b^{-1}\right) \frac{\Delta}{2}-\mathrm{i} r\left(\rho, \sigma_{0}\right)} \\
& =\prod_{\rho \in \Lambda_{R}} \mathrm{~s}_{b}\left(\frac{\mathrm{i}}{2}\left(b+b^{-1}\right)(1-\Delta)-r\left(\rho, \sigma_{0}\right)\right) .
\end{aligned}
$$

Note that here the squashing parameter $b$ serves as a zeta-function regulator in the infinite product formula for the double-sine function (3.39) in the hypermultiplet contribution. For the $\mathcal{N}=1$ adjoint hypermultiplet with $\Delta=1$, the product over $\Lambda_{R}=\triangle$ can be split into contributions from positive-negative pairs of roots and the one-loop contribution is trivial: $Z_{\text {hyp }}\left(S_{b}^{3}\right)=1$.

\subsection{Extension to $S^{1} \times C_{g}$}

One may easily adapt the previous prescriptions to other three-dimensional geometries, which are related to but so far not included in our discussion. As a very basic example, we can consider the three-manifold $M_{3}=S^{1} \times C_{g}$, where the circle $S^{1}$ has radius $\epsilon$ and $C_{g}$ is a closed Riemann surface of genus $g \geq 0$ of area $\operatorname{vol}\left(C_{g}\right)=4 \pi r^{2}$. These manifolds are of course examples of regular fibrations, but admit a different localization locus than those considered thus far.

The fixed point locus in this case consists of $D=0$ and covariantly constant $\sigma$, while the curvature of the gauge connection vanishes along $S^{1}$ and is further constrained by

$$
v\llcorner * F=-2 \sigma,
$$

where $*$ is the Hodge duality operator constructed from the metric of $M_{3}$. Then after gauge fixing, the set of localization equations is solved by connections $A$ whose component 
$A_{\theta}$ along the fibre is a non-vanishing constant $a_{0}$, which we take to lie in a chosen Cartan subalgebra of $\mathfrak{g}$. Expressing the restriction of the curvature to $C_{g}$ in terms of the symplectic form of $C_{g}$ as $F_{C_{g}}=f_{H} \omega_{C_{g}}$, we must impose $\left[a_{0}, f_{H}\right]=0$ to satisfy the condition that $F$ has vanishing components along $S^{1}$. This implies $\sigma=-\frac{1}{2} f_{H}$ is constant, and can be conjugated into the same Cartan subalgebra of $\mathfrak{g}$ as $a_{0}$. This gives a flux $\vec{m} \in \Lambda$ for the restriction $F_{C_{g}}$ to the base $C_{g}$, where $\Lambda$ is a co-root lattice of $\mathfrak{g}$ in the same Cartan subalgebra to which $a_{0}$ belongs. Finally the localization locus is parameterized by $\vec{m}$ and $a_{0}$ as

$$
A=a_{0} \mathrm{~d} \theta+\vec{m} A_{C_{g}} \quad \text { and } \quad \sigma=-\frac{\vec{m}}{r},
$$

where $A_{C_{g}}$ is a monopole gauge field on $C_{g}$ of unit first Chern class, $\mathrm{d} A_{C_{g}}=2 \pi \omega_{C_{g}}$. On the other hand, the circle bundle over $K_{2}=C_{g}$ is trivial, hence the associated line bundle $\mathscr{L}$ has a trivial first Chern class contribution to the index and the topological dependence of the localization formula is solely through the Euler characteristic $\chi\left(C_{g}\right)=2-2 g$.

Including such modifications, the cohomological localization formula yields the oneloop contribution from the vector multiplet as $^{4}$

$$
\begin{aligned}
Z_{\mathrm{vec}}\left(S^{1} \times C_{g}\right) & =\prod_{\alpha \in \triangle} \prod_{n \in \mathbb{Z}}\left(\frac{n}{\epsilon}-\frac{\mathrm{i}(\alpha, \vec{m})}{r}-\left(\alpha, a_{0}\right)\right)^{1-g} \\
& =\prod_{\alpha \in \triangle}\left(2 \sinh \left(\frac{\epsilon\left(\alpha, a_{0}\right)}{2}+\frac{\mathrm{i} \epsilon(\alpha, \vec{m})}{2 r}\right)\right)^{1-g} \\
& =\prod_{\alpha \in \triangle_{+}} \mathrm{e}^{(1-g) \frac{\epsilon}{r}(\alpha, \vec{m})}\left(1-\mathrm{e}^{-\mathrm{i} \epsilon\left(\alpha, a_{0}\right)} \mathrm{e}^{-\frac{\epsilon}{r}(\alpha, \vec{m})}\right)^{1-g} \\
& \times\left(1-\mathrm{e}^{\mathrm{i} \epsilon\left(\alpha, a_{0}\right)} \mathrm{e}^{-\frac{\epsilon}{r}(\alpha, \vec{m})}\right)^{1-g} \\
& =\prod_{\alpha \in \triangle} q^{-\frac{1}{2}(1-g)|(\alpha, \vec{m})|}\left(1-\mathrm{e}^{-\mathrm{i} \epsilon\left(\alpha, a_{0}\right)} q^{|(\alpha, \vec{m})|}\right)^{1-g}
\end{aligned}
$$

where in the last line we defined $q=\mathrm{e}^{-\frac{\epsilon}{r}}$. This reproduces the result of [61, section 2.2], see also [60, section 5.2].

\section{$4 \mathcal{N}=1$ cohomological gauge theories in five dimensions}

In this section we derive one-loop fluctuation determinants in various five-dimensional geometries using the Atiyah-Singer index theorem. We first present the supersymmetric gauge theory and its topologically twisted version, and then derive expressions for the oneloop determinants in the regular and irregular cases separately. We subsequently apply the general formalism to some explicit examples, mainly focusing on five-manifolds of the form $M_{5}=M_{3} \times \Sigma_{h}$, with $M_{3}$ one of the three-dimensional geometries studied in section 3

\footnotetext{
${ }^{4}$ We keep the $n=0$ contribution, and use the overall harmonic dimension factor coming from the ghosts to cancel the Jacobian in the measure for integration over $a_{0}$ in the localized path integral after gauge rotation into a Cartan subalgebra of $\mathfrak{g}$.
} 
and $\Sigma_{h}$ a closed Riemann surface of genus $h$. In this section we adopt the conventions and normalization of [13]. This differs from the rest of the literature on the topic, and in particular the expressions here will only involve anti-holomorphic Dolbeault differentials. ${ }^{5}$

Topologically twisted gauge theories on the five-sphere were studied in [12, 13], which ignited the stream of activity in this area. The next examples considered were the SasakiEinstein manifolds $Y^{p, s}$ in $[18,19,62]$. Sasaki-Einstein manifolds are backgrounds which admit $\mathcal{N}=2$ supersymmetry; see appendix $\mathrm{C}$ for a brief review. In [48], further fivedimensional geometries preserving $\mathcal{N}=1$ and $\mathcal{N}=2$ supersymmetry were obtained, following the idea of $[45,46]$ and adapting it to five dimensions. In particular, one can put an $\mathcal{N}=2$ supersymmetric gauge theory on $S^{3} \times \Sigma_{h}$ and $T^{3} \times \Sigma_{h}$, where $T^{3}$ is a three-dimensional torus. On the other hand, the manifolds $L(p, 1) \times \Sigma_{h}$ only admit $\mathcal{N}=1$ supersymmetry. Further geometries admitting Killing spinor solutions, and hence admitting supersymmetric field theories, were obtained in [49] starting from a holographic setting and taking the rigid limit of supergravity. Sasaki-Einstein manifolds, products $M_{3} \times \Sigma_{h}$ with $M_{3}$ a Seifert three-manifold, and more general U(1) fibrations over products $C_{g} \times \Sigma_{h}$ with $C_{g}$ and $\Sigma_{h}$ Riemann surfaces, possibly with orbifold points, are all examples of manifolds studied in [49].

\subsection{Supersymmetric Yang-Mills theory and its cohomological formulation}

Consider five-dimensional $\mathcal{N}=1$ supersymmetric Yang-Mills theory. We define the theory on flat Euclidean spacetime $\mathbb{R}^{5}$ and then, by coupling it to background supergravity fields, the theory is put on curved manifolds $M_{5}$. For manifolds admitting two Killing spinors, the $\mathcal{N}=2$ vector multiplet is described in the $\mathcal{N}=1$ superspace formalism by an $\mathcal{N}=1$ vector multiplet and an $\mathcal{N}=1$ adjoint hypermultiplet. The required modifications to the supersymmetry transformations are described in detail in [51]. Let $\varepsilon$ be the fivedimensional Killing spinor on $M_{5}$ (see appendix A for our notation), and define the vector field $v$ through $^{6}$

$$
v^{\mu}=\varepsilon^{\dagger} \Gamma^{\mu} \varepsilon
$$

It is a nowhere vanishing Killing vector on $M_{5}$.

Vector multiplet. The five-dimensional $\mathcal{N}=1$ vector multiplet consists of a gauge connection $A$, a scalar $\sigma$, a symplectic Majorana spinor $\lambda$ and an auxiliary real scalar $D$, where $\lambda=\left(\lambda^{I}\right)$ is a $\mathrm{SU}(2)_{R}$ doublet and $D=\left(D_{J}^{I}\right)$ is a $\mathrm{SU}(2)_{R}$ triplet. The supersymmetry

\footnotetext{
${ }^{5}$ The normalization in [13] uses the opposite sign for the contact structure, compared to other literature. After the topological twist, some fields will come with additional minus signs, and in particular a two-form in five dimensions, which is usually taken to be self-dual, becomes anti-self-dual here; with our convention, selfdual 2-forms descend to anti-instantons in four dimensions, and vice versa. In practice, the cohomological complex in five dimensions that we will work with only involves the anti-holomorphic Dolbeault differential, while in previous works (see [53] for a review) the contributions from both holomorphic and anti-holomorphic forms are included. The final results will of course be the same in either convention, but the intermediate steps will slightly differ. The only motivation for our choice is to achieve a unified treatment in three and five dimensions. Furthermore, the topological twist of the hypermultiplets involves only anti-holomorphic forms, so this choice also puts the vector multiplet and the hypermultiplets on the same footing.

${ }^{6}$ This differs by a sign from other definitions in the literature, see Footnote 5.
} 
transformations in flat space are

$$
\begin{aligned}
\mathrm{Q}_{\varepsilon} A_{\mu} & =\mathrm{i} \varepsilon_{I}^{\dagger} \Gamma_{\mu} \lambda^{I}, \\
\mathrm{Q}_{\varepsilon} \sigma & =\varepsilon_{I}^{\dagger} \lambda^{I}, \\
\mathrm{Q}_{\varepsilon} \lambda^{I} & =-\frac{1}{2} \Gamma^{\mu \nu} \varepsilon^{I} F_{\mu \nu}-D^{I}{ }_{J} \varepsilon^{J}+\mathrm{i} \Gamma^{\mu} \varepsilon^{I}\left(\mathrm{D}_{\mu} \sigma\right), \\
\mathrm{Q}_{\varepsilon} D_{I}^{J} & =\mathrm{i} \varepsilon_{I}^{\dagger} \Gamma^{\mu}\left(\mathrm{D}_{\mu} \lambda^{J}\right)-\mathrm{i}\left[\sigma, \varepsilon_{I}^{\dagger} \lambda^{J}\right]+(I \leftrightarrow J),
\end{aligned}
$$

where $F$ is the curvature of the gauge connection $A$ and $\mathrm{D}_{\mu}$ is the covariant derivative, which includes the gauge connection $A$ and also the spin connection when acting on dynamical spinors $\lambda$. Curvature corrections proportional to $\frac{1}{r}$ must be added to these flat space transformations when the field theory is put on $M_{5}$.

At this point we perform the topological twist. We introduce the one-form $\Psi$ and the horizontal anti-self-dual two-form $\chi$ according to ${ }^{7}$

$$
\Psi_{\mu}=\varepsilon_{I}^{\dagger} \Gamma_{\mu} \lambda^{I} \quad \text { and } \quad \chi_{\mu \nu}=\varepsilon_{I}^{\dagger} \Gamma_{\mu \nu} \lambda^{I}-\eta_{\mu} \varepsilon_{I}^{\dagger} \Gamma_{\nu} \lambda^{I}+\eta_{\nu} \varepsilon_{I}^{\dagger} \Gamma_{\mu} \lambda^{I}
$$

where $\eta$ is the one-form dual to the Killing vector $v$. We regard $M_{5}$ as a $\mathrm{U}(1)$ fibration over a compact Kähler manifold $K_{4}$, and when $v$ coincides with the Reeb vector field $\xi$ of the Seifert fibration, then $\eta$ coincides with the K-contact structure $\kappa$ of $M_{5}$. For squashed geometries, however, $\eta \neq \kappa$.

Contact structure and localization locus. The localizing term we add to the action is the standard one:

$$
\mathrm{Q}_{\varepsilon} V \quad \text { with } \quad V=\int_{M_{5}}\left(\mathrm{Q}_{\varepsilon} \lambda\right)^{\dagger} \lambda \mathrm{d} \Omega_{M_{5}},
$$

which in the path integral brings the quantum field theory to the fixed point locus

$$
v\left\llcorner * F=F, \quad \mathrm{D} \sigma=0 \quad \text { and } \quad D=-\sigma \otimes\left(\begin{array}{cc}
1 & 0 \\
0 & -1
\end{array}\right),\right.
$$

where $*$ is the Hodge duality operator constructed from the metric of $M_{5}$.

It is important at this point to stress a major distinction in our setting from that of [12] and subsequent work. When we work with a product of a three-dimensional contact manifold and a Riemann surface, $M_{5}=M_{3} \times \Sigma_{h}$, there is a crucial difference: the contact structure $\kappa$ lives on $M_{3}$, and $\kappa \wedge \mathrm{d} \kappa$ is a volume form on $M_{3}$, as is clear from (2.20), but it need not be a contact structure on $M_{3} \times \Sigma_{h}$. This is important for a choice of compatible metric. For the supersymmetry transformations to be those of a cohomological field theory, one requires the Lie derivative $\mathcal{L}_{v}$ to commute with the Hodge duality operator. Equivalently, we need $v$ to generate an isometry. The Hodge duality operator on $\Omega^{\bullet}\left(M_{3} \times\right.$ $\left.\Sigma_{h}\right)$ takes the form $*_{M_{3} \times \Sigma_{h}}=(-1)^{\bullet} *_{M_{3}} \wedge *_{\Sigma_{h}}$. This is an important simplification in studying the localization locus on product manifolds.

\footnotetext{
${ }^{7}$ We are using the same Greek letter $\chi$ for a two-form here and for a zero-form in section 3 . There should not be any confusion.
} 
The kinetic operator. The supersymmetry transformation squares to $Q_{\varepsilon}^{2}=i L_{\phi}$, with

$$
\mathrm{L}_{\phi}=\mathcal{L}_{v}+\mathcal{G}_{\phi}
$$

the sum of the Lie derivative along $v$ and a gauge transformation with parameter $\phi=$ i $\sigma-v\llcorner A$. Since at the end the integration contour for $\sigma$ must be rotated to the imaginary axis, $\sigma \mapsto \mathrm{i} \sigma_{0}$, we are eventually led to

$$
\phi=-\left(\sigma_{0}+v^{\mu} A_{\mu}^{(0)}\right) \in \mathfrak{g},
$$

with $A^{(0)}$ a connection whose curvature is a solution to the first fixed point equation in (4.5). Setting $A^{(0)}=0$ retains the perturbative partition function, while contributions from non-trivial solutions are related to instantons on the horizontal submanifold.

Hypermultiplets. The field content of a five-dimensional $\mathcal{N}=1$ hypermultiplet consists of a complex scalar $\mathrm{q}=\left(\mathrm{q}_{I}\right)$, which forms an $\mathrm{SU}(2)_{R}$ doublet, and a complex spinor $\psi$. These fields are obtained by combining chiral and anti-chiral complex scalars and Dirac spinors. The supersymmetry transformations are

$$
\begin{aligned}
\mathrm{Q}_{\varepsilon} \mathrm{q}_{I} & =-2 \mathrm{i} \varepsilon_{I}^{\dagger} \psi, \\
\mathrm{Q}_{\varepsilon} \psi & =\Gamma^{\mu} \varepsilon^{I}\left(\mathrm{D}_{\mu} \mathrm{q}_{I}\right)-\sigma \mathrm{q}_{I} \varepsilon^{I} .
\end{aligned}
$$

When coupled to background supergravity fields, additional terms proportional to $\frac{1}{r}$ are to be included.

The topological twist in [13] is then achieved in two steps. First, contract all the $\mathrm{SU}(2)_{R}$ indices, and therefore define the $\mathrm{SU}(2)_{R}$ singlet spinor $\mathrm{q}^{\prime}$ from the scalar $\mathrm{q}_{I}$ as

$$
\mathrm{q}^{\prime}=\mathrm{q}_{I} \varepsilon^{I} .
$$

The square of the supersymmetry transformation, which equals the kinetic operator in the action, is

$$
\mathrm{Q}_{\varepsilon}^{2}=\mathrm{i} \mathrm{L}_{\phi} \quad \text { with } \quad \mathrm{L}_{\phi}=\mathcal{L}_{v}^{\text {spin }}+\mathcal{G}_{\phi},
$$

where we indicated explicitly that the Lie derivative is twisted by the spin connection on $M_{5}$.

The second step consists in defining a $\operatorname{spin}^{c}$ structure on $M_{5}$. For this, in [13] (see also [53, section 3]) the following assumption is made. Let $\eta$ be the dual one-form to the Killing vector field $v$. Then, according to [49], the most general metric on the Seifert fibration $M_{5} \rightarrow K_{4}$ admitting supersymmetry is of the form

$$
\mathrm{d} s_{M_{5}}^{2}=\eta \otimes \eta+\mathrm{d} s_{K_{4}}^{2},
$$

with transverse Hermitian metric on the Kähler surface $K_{4}$. If $\eta$ is proportional to the contact structure defined by the Seifert fibration, then one can define a canonical $\operatorname{spin}^{c}$ structure on $M_{5}$. This condition is equivalent to requiring the orbits of $v$ to be all closed. Manifolds supporting $\mathcal{N}=2$ supersymmetry belong to this class [48], and the index theorem can be applied in that case. 
The $\operatorname{spin}^{c}$ structure identifies, through the action of a representation of the Clifford algebra, spinors with elements of

$$
\Omega_{H}^{(0, \bullet)}\left(M_{5}, \mathfrak{g}\right),
$$

so the hypermultiplet is put in cohomological form. See $[13,53]$ for further details.

After the standard localizing term is added to the action, one has to compute the localization locus. If one considers the trivial solution $A^{(0)}=0$ in the vector multiplet, then the localization locus consists in setting all hypermultiplet fields to zero. It was proven in [18] that this holds for any solution $A^{(0)}$ in the localization locus of the vector multiplet, as long as $\mathrm{d} s_{M_{5}}^{2}$ is a Sasaki-Einstein metric.

Supersymmetric Yang-Mills action at the localization locus. Evaluating the full gauge theory action at the fixed point locus on $M_{5}=M_{3} \times \Sigma_{h}$ gives

$$
S_{\mathrm{cl}}\left(F, \sigma_{0}\right)=\frac{1}{2 g_{\mathrm{YM}}^{2}} \int_{M_{5}}\left((F \wedge * F)+\frac{1}{r}\left(\sigma_{0}, F\right) \wedge \kappa \wedge \mathrm{d} \kappa+\frac{1}{r^{2}}\left(\sigma_{0}, \sigma_{0}\right) \kappa \wedge \mathrm{d} \kappa \wedge \omega_{\Sigma_{h}}\right),
$$

where $g_{\mathrm{YM}}$ is the Yang-Mills coupling constant. Here $\kappa$ is the contact structure on the Seifert three-manifold $M_{3}, \omega_{\Sigma_{h}}$ is the symplectic structure on the Riemann surface $\Sigma_{h}$ and $\frac{1}{2} \kappa \wedge \kappa \wedge \omega_{\Sigma_{h}}$ is the volume form (2.22) on $M_{3} \times \Sigma_{h}$.

\subsection{One-loop determinant of the vector multiplet in a regular background}

After the topological twist performed in section 4.1, all fields of the vector multiplet are in a cohomological form

$$
A \in \Omega^{1}\left(M_{5}, \mathfrak{g}\right), \quad \sigma \in \Omega^{0}\left(M_{5}, \mathfrak{g}\right), \quad \Psi \in \Omega^{1}\left(M_{5}, \mathfrak{g}\right) \quad \text { and } \quad \chi \in \Omega_{H,-}^{2}\left(M_{5}, \mathfrak{g}\right),
$$

where by $\Omega_{H, \pm}^{2}\left(M_{5}, \mathfrak{g}\right)$ we denote the spaces of self-dual and anti-self-dual horizontal twoforms with values in the Lie algebra $\mathfrak{g}$. Here we assume that $M_{5}$ is a regular background, so that contraction by $v$ separates the horizontal and vertical parts of forms. The gauge connection $A$ is our even coordinate and $\chi$ is the odd coordinate on the space of fields. We also have to introduce ghosts, and we refer to $[12,13]$ for the procedure. For our purposes, it suffices to say that these give two even harmonic scalars and two odd scalars.

Gaussian integration of the vector multiplet around the fixed point gives the ratio of fluctuation determinants

$$
h(\phi)=\sqrt{\frac{\left.\operatorname{det} i \mathrm{~L}_{\phi}\right|_{\mathrm{f}}}{\left.\operatorname{det} \mathrm{i} \mathrm{L}_{\phi}\right|_{\mathrm{b}}}}=\sqrt{\frac{\left.\operatorname{det} \mathrm{i} \mathrm{L}_{\phi}\right|_{\Omega_{H,-}^{2}\left(M_{5}, \mathfrak{g}\right)}\left(\left.\operatorname{det} \mathrm{i} \mathrm{L}_{\phi}\right|_{\Omega^{0}\left(M_{5}, \mathfrak{g}\right)}\right)^{2}}{\left.\operatorname{det} \mathrm{i} \mathrm{L}_{\phi}\right|_{\Omega^{1}\left(M_{5}, \mathfrak{g}\right)}\left(\left.\operatorname{det} \mathrm{i} \mathrm{L}_{\phi}\right|_{H^{0}\left(M_{5}, \mathfrak{g}\right)}\right)^{2}}},
$$

where $\left.\right|_{\mathrm{f}}$ (respectively $\left.\right|_{\mathrm{b}}$ ) refers to the operator acting on fermionic (respectively bosonic) fields. Here $H^{0}\left(M_{5}, \mathfrak{g}\right)$ is the space of $\mathfrak{g}$-valued harmonic zero-forms on $M_{5}$, and the differential operator $\mathrm{L}_{\phi}$ is given in (4.6). The numerator of $h(\phi)$ involves the contributions from the fermionic coordinate $\chi$ and the two fermionic ghost coordinates, while the denominator involves the contributions from the bosonic coordinate $A$ and the two bosonic ghost coordinates. 
We split

$$
\begin{aligned}
\Omega_{H}^{2}\left(M_{5}, \mathfrak{g}\right) & =\Omega_{H,+}^{2}\left(M_{5}, \mathfrak{g}\right) \oplus \Omega_{H,-}^{2}\left(M_{5}, \mathfrak{g}\right), \\
\Omega_{H,+}^{2}\left(M_{5}, \mathfrak{g}\right) & =\Omega_{H}^{(2,0)}\left(M_{5}, \mathfrak{g}\right) \oplus \Omega_{H}^{(0,2)}\left(M_{5}, \mathfrak{g}\right) \oplus \Omega_{\text {sympl }}^{(1,1)}\left(M_{5}, \mathfrak{g}\right),
\end{aligned}
$$

where $\Omega_{\text {sympl }}^{(1,1)}\left(M_{5}, \mathfrak{g}\right)$ are the $\mathfrak{g}$-valued horizontal two-forms proportional to the symplectic structure on the base Kähler manifold $K_{4}$. Then

$$
\Omega_{H}^{(1,1)}\left(M_{5}, \mathfrak{g}\right)=\Omega_{\text {sympl }}^{(1,1)}\left(M_{5}, \mathfrak{g}\right) \oplus \Omega_{H,-}^{2}\left(M_{5}, \mathfrak{g}\right) \cong \Omega^{0}\left(M_{5}, \mathfrak{g}\right) \oplus \Omega_{H,-}^{2}\left(M_{5}, \mathfrak{g}\right) .
$$

Here we consider the regular case, in which $v$ is parallel to the Reeb vector field $\xi$. With our choices, $v=-\xi=-\frac{\partial}{\partial \theta}$, where $\theta \in[0,2 \pi r)$ is the coordinate along the circle fibre. We can decompose horizontal forms according to the fibration structure of $M_{5} \rightarrow K_{4}$ as

$$
\Omega_{H}^{(\bullet, \bullet)}\left(M_{5}, \mathfrak{g}\right)=\Omega^{(\bullet \bullet \bullet \bullet)}\left(K_{4}, \mathfrak{g}\right) \oplus \bigoplus_{m \neq 0} \Omega^{(\bullet, \bullet)}\left(K_{4}, \mathscr{L}^{\otimes m} \otimes \mathfrak{g}\right) .
$$

The crucial step now is to recognise that, according to this splitting, the Lie derivative along the Killing vector field $v$ acts on a form $\alpha_{m} \in \Omega^{(\bullet, \bullet)}\left(K_{4}, \mathscr{L}^{\otimes m} \otimes \mathfrak{g}\right)$ as

$$
\mathcal{L}_{v} \alpha_{m}=-\mathcal{L}_{\xi} \alpha_{m}=-\frac{\mathrm{i} m}{r} \alpha_{m}
$$

The fact that the orbits of $v$ coincide with the orbits of the Reeb vector field $\xi$ is essential here. The action of $i \mathrm{~L}_{\phi}$ on each Kaluza-Klein mode labelled by $m \in \mathbb{Z}$ also includes the gauge transformation $\mathcal{G}_{\phi}$, whose eigenmodes are found decomposing the Lie algebra $\mathfrak{g}$ into its root system

$$
\mathfrak{g}=\bigoplus_{\alpha \in \triangle} \mathfrak{g}_{\alpha}
$$

We are therefore ready to evaluate



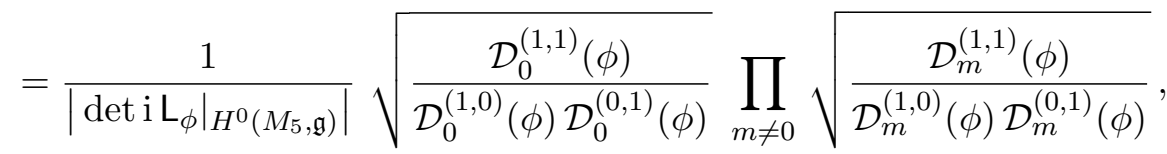

where in the second line we denoted

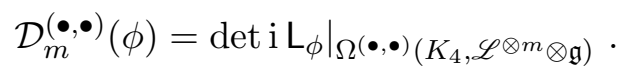

Standard manipulations at this point [13] (see also [12, appendix C]) finally lead to the cohomological localization formula

$$
Z_{\mathrm{vec}}\left(M_{5}\right)=\prod_{\alpha \in \triangle}(\mathrm{i}(\alpha, \phi))^{\frac{1}{12}\left(c_{2}\left(K_{4}\right)+c_{1}\left(K_{4}\right)^{2}\right)-\operatorname{dim} H^{0}\left(M_{5}, \mathbb{R}\right)} \prod_{m \neq 0}\left(\frac{m}{r}+\mathrm{i}(\alpha, \phi)\right)^{\operatorname{index} \bar{\partial}^{(m)}}
$$

For a U(1) bundle $M_{5} \rightarrow C_{g} \times \Sigma_{h}$ over the product of two Riemann surfaces of genera $g$ and $h$, the power of the first multiplicative factor is $(1-g)(1-h)-1$. 


\subsection{One-loop determinant of the vector multiplet in an irregular background}

We now consider the alternative case of an irregular fibration, whereby $v$ does not point along the $\mathrm{U}(1)$ fibre of $M_{5}$. Let $\eta$ be the dual one-form to the Killing vector field $v$. It is an almost contact structure on $M_{5}$; if it is a contact structure, then we are in the situation of section 4.2 above. For the present discussion, we assume that $M_{5}^{g, h} \rightarrow C_{g} \times \Sigma_{h}$ is a $\mathrm{U}(1)$ fibration over a direct product of two Riemann surfaces, both compact and closed. Rotations along the circle fibre are assumed to act freely ${ }^{8}$ on $M_{5}^{g, h}$. This means that, although the gauge theory could be put on $M_{5}^{g, h}$ preserving $\mathcal{N}=1$ supersymmetry when $M_{5}^{g, h} / \mathrm{U}(1)$ admits orbifold points [49], the procedure we describe below does not apply to that case. Since we are in the irregular setting, we need an additional U(1) isometry on $C_{g} \times \Sigma_{h}$. In practice, this restricts our considerations to $C_{0}=S^{2}$ or $C_{1}=T^{2}$.

Most of the procedure is exactly the same as in section 4.2, particularly the decomposition of differential forms in terms of the Reeb vector field $\xi$. Nonetheless, we have to face two problems. First, as for the irregular three-dimensional case, we have to bear in mind that forms $\alpha_{m} \in \Omega^{(\bullet, \bullet)}\left(C_{g} \times \Sigma_{h}, \mathscr{L}^{\otimes m} \otimes \mathfrak{g}\right)$ are no longer eigenmodes of $\mathcal{L}_{v}$. The other important issue is that now the conditions following from the definition of the two-form $\chi$,

$$
v\llcorner\chi=0 \quad \text { and } \quad v\llcorner * \chi=-\chi,
$$

cannot be interpreted as saying that $\chi$ is a horizontal anti-self-dual two-form. If we express $v$ as a linear combination

$$
v=a_{1} \xi+a_{2} \widetilde{\xi}
$$

where $\xi$ is the Reeb vector field and $\widetilde{\xi}$ is a vector field orthogonal to $\xi$ generating a U(1) action, we find that the vertical part of $\chi$ may not vanish, but it lies in the subspace orthogonal to $\widetilde{\xi}$. However, more is true: we can decompose $\chi$ in terms of a two-form $\chi_{T}$ and a one-form $\chi_{P}$. Explicitly

$$
\chi=\left(\kappa-\frac{a_{1}}{a_{2}} \widetilde{\kappa}\right) \wedge \chi_{P}+\chi_{T}
$$

where $\kappa$ is the contact structure and $\widetilde{\kappa}$ is the one-form dual to $\widetilde{\xi}$, and with further antiself-duality relations imposed on $\chi_{P}$ and $\chi_{T}$. In the end, we are left with the same number of degrees of freedom as for a horizontal anti-self-dual two-form. From the more geometric perspective of transverse holomorphic foliations, the most natural point of view is to consider now the index of a new Dolbeault-like operator $\widetilde{\partial}^{(m)}$, whose cohomological complex is a deformation of the cohomological complex of the regular case according to the deformation of the transverse holomorphic foliation of $M_{5}^{g, h}$ as described in [59, section 5] (see also [59, section 7] and [50, section 5] for a discussion about the particular case of ellipsoids).

At this point, we can again follow the approach of [14] and extend it to five dimensions. The action of the Reeb vector field is free, and we can reduce the computations to the

\footnotetext{
${ }^{8}$ The Sasaki-Einstein manifolds $Y^{p, s}$ do not belong to this class, see appendix C and section 2.4.
} 
quotient space $C_{g} \times \Sigma_{h}$. In this way we arrive at the cohomological localization formula

$$
Z_{\text {vec }}\left(M_{5}^{g, h}\right)=\prod_{\alpha \in \triangle}(\mathrm{i}(\alpha, \phi))^{g h-g-h} \prod_{f} \prod_{m \neq 0}\left(\frac{m}{\epsilon_{f}}+\mathrm{i}(\alpha, \phi)\right)^{\frac{1}{2} \operatorname{index} \bar{\partial}^{(m)}}
$$

with an extra product over the fixed points of the additional U(1) action on $C_{g} \times \Sigma_{h}$. The length parameter $\epsilon_{f}$ is the radius of the circle fibre over the fixed point labelled by $f$.

\section{One-loop determinant of the vector multiplet on $M_{3} \times \Sigma_{h}$}

We will now specialise the present discussion to product manifolds $M_{5}^{g, h}=M_{3} \times \Sigma_{h}$, where the classification reduces to the discussion of [46] about the orbits of the three-dimensional Killing vector field on $M_{3}$. We shall explicitly compute the Atiyah-Singer index in this case. The Kähler surface $K_{4}=C_{g} \times \Sigma_{h}$ is endowed with the product Kähler structure $\omega=\omega_{C_{g}}+\omega_{\Sigma_{h}}$ and the U(1)-bundle projection $\pi$ is the product of the Seifert fibration $M_{3} \rightarrow C_{g}$ and the identity map on $\Sigma_{h}$. The integer cohomology of $K_{4}$ has generators $\left[\omega_{C_{g}}\right] \in H^{2}\left(C_{g}, \mathbb{Z}\right)$ and $\left[\omega_{\Sigma_{h}}\right] \in H^{2}\left(\Sigma_{h}, \mathbb{Z}\right)$ in this case, and the first Chern class of the line bundle $\mathscr{L} \rightarrow K_{4}$ associated to the circle fibration is given by $c_{1}(\mathscr{L})=\operatorname{deg}(\mathscr{L}) \omega_{C_{g}}$. One has $c\left(T^{1,0} \Sigma_{h}\right)=1+\chi\left(\Sigma_{h}\right) \omega_{\Sigma_{h}}=1+c_{1}\left(T^{1,0} \Sigma_{h}\right)$ with $\chi\left(\Sigma_{h}\right)=2-2 h$ the Euler characteristic of the Riemann surface $\Sigma_{h}$, and similarly for $C_{g}$. The total Chern class is thus

$$
\begin{aligned}
c\left(T^{1,0} K_{4}\right) & =c\left(T^{1,0} C_{g}\right) \wedge c\left(T^{1,0} \Sigma_{h}\right) \\
& =\left(1+\chi\left(C_{g}\right) \omega_{C_{g}}\right) \wedge\left(1+\chi\left(\Sigma_{h}\right) \omega_{\Sigma_{h}}\right) \\
& =1+2\left((1-g) \omega_{C_{g}}+(1-h) \omega_{\Sigma_{h}}\right)+4(1-g)(1-h) \omega_{C_{g}} \wedge \omega_{\Sigma_{h}} \\
& =1+c_{1}\left(T^{1,0} K_{4}\right)+c_{2}\left(T^{1,0} K_{4}\right),
\end{aligned}
$$

and the corresponding Todd class is

$$
\begin{aligned}
\operatorname{Td}\left(T^{1,0} K_{4}\right) & =1+\frac{1}{2} c_{1}\left(T^{1,0} K_{4}\right)+\frac{1}{12}\left(c_{1}\left(T^{1,0} K_{4}\right) \wedge c_{1}\left(T^{1,0} K_{4}\right)+c_{2}\left(T^{1,0} K_{4}\right)\right) \\
& =1+(1-g) \omega_{C_{g}}+(1-h) \omega_{\Sigma_{h}}+(1-g)(1-h) \omega_{C_{g}} \wedge \omega_{\Sigma_{h}} .
\end{aligned}
$$

Using $c_{1}\left(\mathscr{L}^{\otimes m}\right)=m \operatorname{deg}(\mathscr{L}) \omega_{C_{g}}$, the corresponding Chern character is found to be

$$
\operatorname{ch}\left(\mathscr{L}^{\otimes m}\right)=1+c_{1}\left(\mathscr{L}^{\otimes m}\right)+\frac{1}{2} c_{1}\left(\mathscr{L}^{\otimes m}\right) \wedge c_{1}\left(\mathscr{L}^{\otimes m}\right)=1+m \operatorname{deg}(\mathscr{L}) \omega_{C_{g}} .
$$

The index of the Dolbeault complex in this case is thus given by

$$
\begin{aligned}
\operatorname{index} \bar{\partial}^{(m)}= & \int_{K_{4}}\left(1+m \operatorname{deg}(\mathscr{L}) \omega_{C_{g}}\right) \wedge\left(1+(1-g) \omega_{C_{g}}+(1-h) \omega_{\Sigma_{h}}\right. \\
& \left.+(1-g)(1-h) \omega_{C_{g}} \wedge \omega_{\Sigma_{h}}\right) \\
& =\int_{K_{4}}((1-g)(1-h)+m(1-h) \operatorname{deg}(\mathscr{L})) \omega_{C_{g}} \wedge \omega_{\Sigma_{h}} \\
& =(1-h) \int_{C_{g}}(m \operatorname{deg}(\mathscr{L})+1-g) \omega_{C_{g}} \\
& =(1-h)[m \operatorname{deg}(\mathscr{L})+1-g] .
\end{aligned}
$$


The term in square brackets is the index of the twisted Dolbeault complex associated to the circle bundle $M_{3} \rightarrow C_{g}$, and we finally get the localization formula

$$
Z_{\mathrm{vec}}\left(M_{3} \times \Sigma_{h}\right)=Z_{\mathrm{vec}}\left(M_{3}\right)^{1-h} \prod_{\alpha \in \triangle_{+}}(\alpha, \mathrm{i} \sigma)^{-2 h}
$$

Again, the multiplicative factor in the general localization formula, which includes also contributions from the ghosts, is essential for cancelling the denominator. This formula can be extended to the case in which $C_{g}$ has orbifold points, as reviewed in [50, section 3.5].

Notice that the localization formula for the one-loop determinants on $M_{3} \times \Sigma_{h}$ lifts the perturbative three-dimensional partition function to the perturbative five-dimensional partition function. However, while the full (non-perturbative) partition function on $M_{3}$ receives contributions from flat connections on $M_{3}$, the full partition function on $M_{3} \times \Sigma_{h}$ includes connections $A^{(0)}$ that descend to instantons on $C_{g} \times \Sigma_{h}$. The moduli spaces over which we integrate are different. In fact, the pullback to $M_{5}$ of flat connections on $M_{3}$ are not generally solutions to the fixed point equation (4.5). This is a major difference from the partially twisted theory, in which the BPS configurations decompose into flat connections on $M_{3}$ and unconstrained connections on $\Sigma_{h}$.

\subsection{One-loop determinant of a hypermultiplet}

For the contribution of a hypermultiplet, we will only consider a regular background here, due to the issues encountered with the vector multiplet discussed in section 4.3. Furthermore, we consider only the one-loop determinant in the perturbative partition function, that is we set $A^{(0)}=0$, hence $\phi=-\sigma_{0}$. We want to calculate the ratio of fluctuation determinants

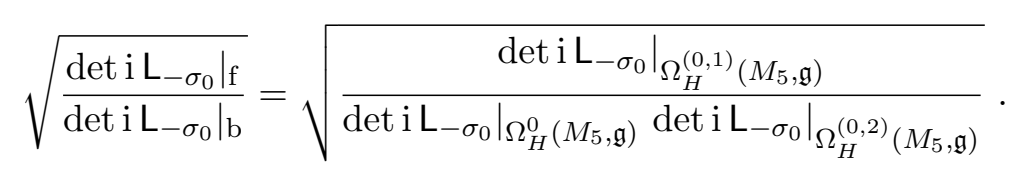

Applying the same strategy as with the vector multiplet, that is, decomposing the horizontal forms according to the tensor powers of the line bundle $\mathscr{L}$ associated to the $\mathrm{U}(1)$ fibration, one arrives at the cohomological localization formula

$$
Z_{\text {hyp }}^{\text {pert }}\left(M_{5}\right)=\prod_{\rho \in \Lambda_{R}} \prod_{m \in \mathbb{Z}}\left(\frac{m+\Delta}{r}-\mathrm{i}\left(\rho, \sigma_{0}\right)\right)^{-\operatorname{index} \bar{\partial}^{(m)}}
$$

We briefly comment on the cohomological formulation of the hypermultiplet in a squashed or ellipsoid background. The topological twist depends, in general, on the geometric data. However, as discussed in [51] and also in [53, section 3.3], we can turn on the squashing and continuously deform the contact structure, so that the Reeb vector field associated to the new contact structure stays parallel to the Killing vector field $v$. Then, the cohomological localization applies to the vector multiplet, although using a notion of 'horizontal' which differs from that on the round manifold we started with. For the topological twist of the hypermultiplet, however, a choice of $\operatorname{spin}^{c}$ structure is needed, and hence additional assumptions on the geometry of the base of the $\mathrm{U}(1)$ fibration are required, usually 
that it is Kähler-Einstein. The pragmatic solution of [51] was to take the cohomological form of the hypermultiplet as a definition in a squashed Sasaki-Einstein geometry. This is not, however, a continuous deformation of the hypermultiplet in the round geometry, and we do not follow this strategy here.

\subsection{Perturbative partition functions}

We now come to the first applications of our cohomological localization formulas in five dimensions. Pan proved in [48] that the product manifolds $M_{3} \times \Sigma_{h}$ admit $\mathcal{N}=2$ supersymmetry if $M_{3}=S^{3}$ or $M_{3}=T^{3}$, the sphere or the torus. The second choice is not included in the discussion so far, and we will consider it later in section 4.8. We will work out the full perturbative $\mathcal{N}=2$ partition functions on $S^{3} \times \Sigma_{h}$. We shall then write down the perturbative $\mathcal{N}=1$ partition functions on more general product five-manifolds $M_{3} \times \Sigma_{h}$.

\section{$\mathcal{N}=2$ perturbative partition functions on $S^{3} \times \Sigma_{h}$}

Regard the three-sphere $S^{3}$ as the Hopf fibration of degree one over $C_{0}=S^{2}$. We use the localization formula (4.32) with $A^{(0)}=0$ together with (4.34) which gives

$$
Z_{\text {vec }}^{\text {pert }}\left(S^{3} \times \Sigma_{h}\right)=Z_{\text {vec }}\left(S^{3}\right)^{1-h} \quad \text { and } \quad Z_{\text {hyp }}^{\text {pert }}\left(S^{3} \times \Sigma_{h}\right)=Z_{\text {hyp }}\left(S^{3}\right)^{1-h} .
$$

The full perturbative partition function is given by taking the product of the one-loop vector multiplet determinant with products of the one-loop hypermultiplet determinants over all $\mathcal{N}=2$ hypermultiplets $a$ of conformal dimensions $\Delta_{a}$ in representations $R_{a}$ of the gauge group $G$. We then multiply by the Boltzmann weight of the classical action (4.13) evaluated at the trivial solution $A^{(0)}=0$, and integrate over the remaining scalar moduli $\sigma_{0} \in \mathfrak{g}$ using the localization formulas of section 3.5. We can conjugate $\sigma_{0}$ into a Cartan subalgebra $\mathfrak{t} \subset \mathfrak{g}$ and use the Weyl integral formula to perform the resulting integral with the measure

$$
\mathrm{d} \mu\left(\sigma_{0}\right)=\mathrm{d} \sigma_{0} \prod_{\alpha \in \triangle_{+}}\left(\alpha, \sigma_{0}\right)^{2}
$$

where $\mathrm{d} \sigma_{0}$ is the Lebesgue measure on $\mathfrak{t}=\mathbb{R}^{\operatorname{rank}(G)}$. The applicability of the Weyl integral formula is restricted to elements $\sigma_{0} \in \mathfrak{t}$ for which the determinant in (4.36) is nonvanishing; these are called regular elements, and they form an open dense subset $\mathfrak{t}_{\text {reg }} \subset \mathfrak{t}$. After cancelling the Jacobian in the integration measure with the denominator of the vector multiplet one-loop determinant, we obtain the perturbative partition function in the background of [48]:

$$
\begin{aligned}
Z_{\mathcal{N}=2}^{\text {pert }}\left(S^{3} \times \Sigma_{h}\right)=\int_{\mathfrak{t}_{\text {reg }}} \mathrm{d} \tilde{\sigma} \mathrm{e}^{-\frac{4 \pi^{2} \mathrm{vol}\left(\Sigma_{h}\right)}{r g_{\mathrm{YM}}^{2}}(\tilde{\sigma}, \tilde{\sigma})} & \prod_{\alpha \in \triangle_{+}} \sinh (\pi(\alpha, \tilde{\sigma}))^{2-2 h} \\
& \times \prod_{a} \prod_{\rho_{a} \in \Lambda_{R_{a}}} \mathrm{~s}_{1}\left(\mathrm{i}\left(1-\Delta_{a}\right)-\left(\rho_{a}, \tilde{\sigma}\right)\right)^{1-h}
\end{aligned}
$$

where we defined the variable $\tilde{\sigma}=r \sigma_{0}$ in the Cartan subalgebra $\mathfrak{t}_{\text {reg }} \subset \mathfrak{g}$. The $\mathcal{N}=1$ partition function on $S^{3} \times \Sigma_{h}$ without matter and including instanton contributions will be analysed in section 5.1. 
$\mathcal{N}=1$ perturbative partition functions on $M_{3} \times \Sigma_{h}$

We shall now consider the $\mathcal{N}=1$ perturbative partition functions on more general $\mathrm{U}(1)$ fibrations over $C_{g} \times \Sigma_{h}$, where $C_{g}$ is a Riemann surface of genus $g$, focusing on the case $M_{5}=M_{3} \times \Sigma_{h}$ where only $M_{3} \rightarrow C_{g}$ is fibered over $C_{g}$. Proceeding as above using the index formula (4.31), we arrive at

$$
\begin{aligned}
Z_{\mathcal{N}=1}^{\mathrm{pert}}\left(M_{3} \times \Sigma_{h}\right)=\int_{\mathrm{t}_{\mathrm{reg}}} \mathrm{d} \tilde{\sigma} \mathrm{e}^{-\frac{\pi \operatorname{vol}\left(C_{g}\right) \operatorname{vol}\left(\Sigma_{h}\right)}{r^{3} g_{\mathrm{YM}}^{2}}(\tilde{\sigma}, \tilde{\sigma})} & \prod_{\alpha \in \Delta_{+}} \sinh (\pi(\alpha, \tilde{\sigma}))^{2(1-h)(1-g)} \\
& \times \prod_{a} \prod_{\rho_{a} \in \Lambda_{R_{a}}} \mathrm{~s}_{1}\left(\mathrm{i}\left(1-\Delta_{a}\right)-\left(\rho_{a}, \tilde{\sigma}\right)\right)^{(1-h) \operatorname{deg}(\mathscr{L})} .
\end{aligned}
$$

The products $L(p, 1) \times \Sigma_{h}$ are particular examples [48] with $C_{0}=S^{2}$, for which $\operatorname{deg}(\mathscr{L})=p$. The formalism should also apply when $M_{3}$ is a more general Seifert homology sphere which admits a contact structure, and in particular for the lens spaces $M_{3}=L(p, s)$. It would also be interesting to extend the formalism to the case in which $\Sigma_{h}$ has punctures.

\subsection{Contact instantons and their pushdown to four dimensions}

We shall now work out solutions to the fixed point equation (4.5) on $M_{5}=M_{3} \times \Sigma_{h}$, and then study their pushdown to four dimensions.

Regular fibrations. We first focus on regular Seifert manifolds. We want to solve the equation

$$
v\llcorner * F=F
$$

on $M_{3} \times \Sigma_{h}$, where $v=-\xi$ is the Killing vector field with $\xi$ the Reeb vector field of the Seifert fibration $M_{3} \rightarrow C_{g}$. On K-contact five-manifolds, the solutions to this equation are refered to as contact instantons [12], and their moduli spaces are studied in [63]. We can rewrite (4.39) as

$$
* F=\kappa \wedge F
$$

or equivalently

$$
v\left\llcorner F=0 \quad \text { and } \quad F_{H,-}=0,\right.
$$

where $F_{H, \pm}$ denote the self-dual and anti-self-dual horizontal parts of the curvature twoform $F$. Let us decompose the gauge connection as

$$
A=A_{\theta} \kappa+A_{H},
$$

where $\kappa$ is (minus) the contact structure on $M_{3}$ dual to $v$, so that

$$
F=\left(A_{\theta} \mathrm{d} \kappa+\mathrm{d} A_{\theta} \wedge \kappa+\mathrm{d} A_{H}\right)-\mathrm{i}\left(A_{H} \wedge A_{H}+\left[A_{\theta}, A_{H}\right] \wedge \kappa\right) .
$$

We partly follow the treatment of [13, section 3.2]. The first equation $v\llcorner F=0$ reads

$$
\mathrm{D}_{H} A_{\theta}=\mathrm{d} A_{\theta}+\mathrm{i}\left[A_{H}, A_{\theta}\right]=0
$$


so $A_{\theta}$ is covariantly constant along $C_{g} \times \Sigma_{h}$. At this point, it is useful to prove that both the $\mathfrak{g}$-valued function $A_{\theta}$ and one-form $A_{H}$ are invariant under translations along the fibre, generated by $v$. For this, we choose the gauge ${ }^{9}$

$$
\mathcal{L}_{v} A=0 \text {. }
$$

Then

$$
\mathcal{L}_{v} A_{\theta}=v\left\llcorner\mathrm{~d} A_{\theta}=-\mathrm{i} v\left\llcorner\left[A_{H}, A_{\theta}\right]=0 \quad \text { and } \quad \mathcal{L}_{v} A_{H}=v\left\llcorner\mathrm{~d} A_{H}+\mathrm{d}\left(v\left\llcorner A_{H}\right)=0,\right.\right.\right.\right.
$$

where $v\left\llcorner\mathrm{~d} A_{H}=0\right.$ follows from the gauge fixing condition (4.45).

From (4.44) it follows that the curvature $F$ has only a horizontal part given by

$$
F=F_{H}=A_{\theta} \mathrm{d} \kappa+\mathrm{d} A_{H}-\mathrm{i} A_{H} \wedge A_{H} .
$$

At this point we use the second equation $F_{H,-}=0$. The surviving self-dual part belongs to the vector space

$$
F_{H,+} \in \Omega_{\text {sympl }}^{(1,1)}\left(C_{g} \times \Sigma_{h}, \mathfrak{g}\right) \oplus \Omega^{(2,0)}\left(C_{g} \times \Sigma_{h}, \mathfrak{g}\right) \oplus \Omega^{(0,2)}\left(C_{g} \times \Sigma_{h}, \mathfrak{g}\right) .
$$

This implies that $\mathrm{d} A_{H}$ is proportional to the Kähler two-form $\omega_{C_{g}}+\omega_{\Sigma_{h}}$ on the base, and recalling the relation (2.21) between $\mathrm{d} \kappa$ and the Kähler form on $C_{g} \times \Sigma_{h}$, altogether we arrive at a curvature which is of the form

$$
F=f_{H}\left(\omega_{C_{g}}+\omega_{\Sigma_{h}}\right)+F^{(2,0)}+F^{(0,2)},
$$

where

$$
F^{(2,0)}=-\mathrm{i}\left[\left(A_{H}\right)_{y},\left(A_{H}\right)_{z}\right] \mathrm{d} y \wedge \mathrm{d} z \quad \text { and } \quad F^{(0,2)}=-\mathrm{i}\left[\left(A_{H}\right)_{\bar{y}},\left(A_{H}\right)_{\bar{z}}\right] \mathrm{d} \bar{y} \wedge \mathrm{d} \bar{z},
$$

and we have chosen local complex coordinates $(y, \bar{y}) \in C_{g}$ and $(z, \bar{z}) \in \Sigma_{h}$. The function $f_{H} \in \Omega^{0}\left(C_{g} \times \Sigma_{h}, \mathfrak{g}\right)$ is a purely four-dimensional quantity.

To summarise, we arrive at a solution $A=A_{\theta} \kappa+A_{H}$ on $M_{3} \times \Sigma_{h}$, where $A_{H}$ is a connection on $C_{g} \times \Sigma_{h}$, and $A_{\theta}$ is a $\mathfrak{g}$-valued function which is constant along the fibre and covariantly constant on $C_{g} \times \Sigma_{h}$ with respect to $A_{H}$. The curvature $F$ of $A$ lives on $C_{g} \times \Sigma_{h}$ and is self-dual (from the five-dimensional point of view). The Yang-Mills action evaluated at these connections gives

$$
\begin{aligned}
S_{\mathrm{YM}}(F) & =\frac{1}{2 g_{\mathrm{YM}}^{2}} \int_{M_{5}}(F \wedge * F) \\
& =\frac{1}{2 g_{\mathrm{YM}}^{2}} \int_{M_{5}} \kappa \wedge\left(F_{H,+} \wedge F_{H,+}\right) \\
& =-\frac{\pi r}{g_{\mathrm{YM}}^{2}} \int_{C_{g} \times \Sigma_{h}}\left(F_{H,+} \wedge F_{H,+}\right) \\
& =\frac{8 \pi^{3} r(\vec{m}, \vec{n})}{g_{\mathrm{YM}}^{2}},
\end{aligned}
$$

\footnotetext{
${ }^{9}$ We avoid formal considerations involved in the gauge fixing procedure. The details are exactly as in $[12,13]$.
} 
where we integrated over the circular fibre, of radius $r$, and used the fact that $F_{H,+}$ is independent of the fibre direction. The integer vectors $\vec{m}, \vec{n} \in \mathbb{Z}^{\operatorname{rank}(G)}$ are the gauge fluxes through $C_{g}$ and $\Sigma_{h}$, respectively, which can be identified with weights of the Lie algebra $\mathfrak{g}$. Then $(\vec{m}, \vec{n}) \in \mathbb{Z}$ is proportional to the second Chern character ${ }^{10} \operatorname{ch}_{2}(P) \in$ $H^{4}\left(C_{g} \times \Sigma_{h}, \mathbb{Q}\right)=H^{2}\left(C_{g}, \mathbb{Q}\right) \otimes H^{2}\left(\Sigma_{h}, \mathbb{Q}\right)$ of the principal $G$-bundle $P \rightarrow C_{g} \times \Sigma_{h}$ on which $A_{H}$ is a connection. This reduction along the Seifert fibre of $M_{3}$ may be thought of as a lift of the technique of $[56,64]$.

Five-dimensional gauge theories also have a topological global U(1) inst symmetry [27], with conserved current

$$
J_{\text {inst }}=*(F \wedge F) .
$$

The $\mathrm{U}(1)_{\text {inst }}$ charge of the current $J_{\text {inst }}$ is the instanton number computed from $F$. The derivation given above makes it clear that this topological symmetry is related to the $\mathrm{U}(1)$ invariance under rotation of the Seifert fibre.

Pushdown. Let us now describe the pushdown of these solutions. The localization of the supersymmetric gauge theory onto connections constant along the fibre, whose curvature descends to four dimensions, is reminiscent of the framework of [65], where the topologically twisted theory on $S^{2} \times S^{2}$ was studied. In particular, the vertical component $A_{\theta}$ of the gauge field $A$ is covariantly constant on the four-dimensional base manifold $C_{g} \times \Sigma_{h}$, thus $A_{\theta}$ is a scalar field on $C_{g} \times \Sigma_{h}$ constrained in exactly the same way as the scalar $\sigma$. Following [66], we can redefine our vector multiplet and hypermultiplets in terms of four-dimensional supersymmetry multiplets. The real scalar $\sigma$ is combined with the vertical component $A_{\theta}$ to give a complex scalar $\phi=\mathrm{i} \sigma-v\llcorner A$, together with a purely four-dimensional gauge connection $A_{H}$. This reduction brings the $\mathcal{N}=1$ five-dimensional vector multiplet down to the $\mathcal{N}=2$ four-dimensional vector multiplet. Similar manipulations can be done for the hypermultiplets. The five-dimensional Majorana spinor $\varepsilon$ breaks down into one left and one right chiral four-dimensional Killing spinor.

In [65, sections 3 and 4], it is explained how to topologically twist the $\mathcal{N}=2$ gauge theory on any four-dimensional manifold admitting a $U(1)$ isometry. We can then simply borrow their results: the fixed point equations in four dimensions are

$$
[F, \phi]=\left[F, \phi^{\dagger}\right]=\left[\phi, \phi^{\dagger}\right]=0, \quad w\left\llcorner\mathrm{D}_{H} \phi^{\dagger}=0 \quad \text { and } \quad w\llcorner F-\mathrm{id} \phi=0,\right.
$$

where $w$ is the vector field used for the twist. The vanishing Lie brackets imply that we can conjugate all fields $A_{H}, \sigma$ and $v\llcorner A$ into the same Cartan subalgebra of $\mathfrak{g}$ simultaneously. This means that covariantly constant scalars $\sigma$ can be taken to be constant. As pointed out in [65, section 4.3], to obtain the full partition function one should include not only the sum over gauge fluxes $\vec{m}$ and $\vec{n}$ through the two surfaces $C_{g}$ and $\Sigma_{h}$ (which we have equivalently obtained from direct computations in five dimensions), but also the Nekrasov partition functions which sum over point-like instantons corresponding to the fixed points of the action of the maximal torus of the symmetry group given by the direct product of the gauge group with the isometry group.

\footnotetext{
${ }^{10}$ We use Künneth's theorem here.
} 
To explicitly compute the instanton contributions, we could take $C_{0}=\Sigma_{0}=S^{2}$, where our result coincides with that of [16, section 2]; they proceed in the other direction, starting from the theory on $S^{2} \times S^{2}$ with $\Omega$-background and then lifting it to $S_{b}^{3} \times S^{2}$. The other geometry where instanton contributions are tractable is a reduction onto $S^{2} \times T^{2}$. In this case, the $\mathcal{N}=2$ four-dimensional theory was analyzed in [67, 68] (see also [69]), which in turn computes the elliptic genus of an $\mathcal{N}=(2,2)$ gauge theory on the torus $T^{2}$ [70]. ${ }^{11}$ While contributions from four-dimensional point-like instantons on $C_{g} \times \Sigma_{h}$ are hard to compute in more general geometries, we may hope to recover the full answer from a resurgent analysis, as explained in [71] for $S^{4}$. Borel summability of the perturbation series for $\mathcal{N}=2$ theories on $S^{4}$ and $\mathcal{N}=1$ theories on $S^{5}$ (both possibly squashed) has been studied in [72]. We show in section 4.7 below how to adapt this argument to some examples in the present setting.

Irregular fibrations. The irregular case is more subtle. In this case, we may attempt to proceed as in the regular case, but now with the Killing vector $v$ no longer pointing along the fibre direction. In other words, the equation

$$
v\llcorner * F=F
$$

cannot be interpreted in terms of horizontal and anti-self-dual components. Instead, we can decompose the gauge field as

$$
A=A_{\eta} \eta+A_{T}
$$

where $\eta$ is the one-form dual to $v, A_{\eta}$ is the component of the gauge connection along the direction of the isometry generated by $v$, and $A_{T}$ is the transverse gauge connection. That is, we replace the notion of horizontal with that of transverse, which is natural in the present context [49]. Then the condition $v\llcorner F=0$, necessary to fulfill (4.54), can be solved in an analogous way as for the regular case, leading to

$$
\mathrm{D}_{T} A_{\eta}=\mathrm{d} A_{\eta}+\mathrm{i}\left[A_{T}, A_{\eta}\right]=0 \quad \text { and } \quad \mathcal{L}_{v} A_{\eta}=0=\mathcal{L}_{v} A_{T} .
$$

From the analysis of the regular case above, it is clear that we can again pushdown the theory to four dimensions. However, this time we do not reduce to the base $C_{g} \times \Sigma_{h}$ of the Seifert fibration, but instead to the submanifold transverse to $\eta$ (equivalently, the submanifold orthogonal to $v$ ). This is what one expects by the construction of [49].

\subsection{Borel summability}

Consider the perturbative contribution to the partition function of $\mathcal{N}=1$ gauge theory on $S^{3} \times \Sigma_{h}$ with gauge group $G=\mathrm{U}(N)$ and massless hypermultiplets, with $N_{f}$ in the fundamental representation and $N_{\bar{f}}$ in the anti-fundamental representation. The perturbative partition function is formally the same as the $\mathcal{N}=2$ perturbative partition function

\footnotetext{
${ }^{11}$ The case of interest to us is described in [67, section 5.1], but without gauging the flavour symmetry. In the two-dimensional theory, this corresponds to turning off the moduli associated to background fields, and in particular the two-dimensional R-symmetry is not gauged.
} 
computed in section 4.5, but now allowing a more general assignment of charges $\Delta_{f}$ and $\Delta_{\bar{f}}$ for the hypermultiplets. Changing integration variables to hyperspherical coordinates

$$
\tilde{\sigma}_{j}=\sqrt{\tau} x_{j} \quad \text { with } \quad \sum_{j=1}^{N} x_{j}^{2}=1 \quad \text { and } \quad 0 \leq \tau<\infty
$$

as in [73], we can rewrite the partition function (4.37) in the form

$$
Z_{\mathcal{N}=2}^{\text {pert }}\left(S^{3} \times \Sigma_{h}\right)=\int_{0}^{\infty} \mathrm{d} \tau \mathrm{e}^{-\frac{\tau}{\gamma}} Y_{N}(\tau)
$$

where $\gamma^{-1}=\frac{4 \pi^{2}}{r g_{\mathrm{YM}}^{2}} \operatorname{vol}\left(\Sigma_{h}\right)$ and

$$
\begin{aligned}
Y_{N}(\tau)= & \frac{\tau^{\left(N^{2}-2\right) / 2}}{2} \int_{\mathbb{R}^{N}} \mathrm{~d} x \delta\left(\sum_{j=1}^{N} x_{j}^{2}-1\right) \prod_{1 \leq j<k \leq N}\left(x_{j}-x_{k}\right)^{2} \\
& \times\left(\prod_{1 \leq j<k \leq N} \frac{\sinh \left(\pi \sqrt{\tau}\left(x_{j}-x_{k}\right)\right)^{2}}{\tau\left(x_{j}-x_{k}\right)^{2}}\right)^{1-h} \\
& \times\left(\prod_{j=1}^{N} \mathrm{~s}_{1}\left(\mathrm{i}\left(1-\Delta_{f}\right)-\sqrt{\tau} x_{j}\right)^{N_{f}} \mathrm{~s}_{1}\left(\mathrm{i}\left(1-\Delta_{\bar{f}}\right)+\sqrt{\tau} x_{j}\right)^{N_{\bar{f}}}\right)^{1-h} .
\end{aligned}
$$

This form is suitable to study the Borel summability of the partition function $\mathcal{Z}_{\mathcal{N}=1}^{\text {pert }}\left(S^{3} \times\right.$ $\Sigma_{h}$ ), and of the $\mathcal{N}=2$ theory as a particular case. In fact, this expression is exactly as in [73], except for the powers $1-h$. For genus $h=0$ Borel summability follows from [73] and for $h=1$ it is straightforward. The proof of Borel summability at $h>1$ is almost exactly as in [73] except for minor changes in the proof of the uniform convergence. As in [73], the argument can be extended to include $N_{\text {adj }}$ adjoint hypermultiplets.

For the particularly simple case $\Sigma_{0}=S^{2}$, we can extend this analysis of Borel summability beyond the perturbative sector. It suffices to notice that non-perturbative contributions to the $\mathcal{N}=1$ partition function on $S^{3} \times S^{2}$ come from four-dimensional (anti)instantons on $S^{2} \times S^{2}$. The $\mathcal{N}=2$ instanton partition function was obtained in [65], and it coincides with the instanton partition function on $S^{4}[65,72]$. Therefore for $\mathrm{U}(N)$ gauge theory with $N_{f}$ hypermultiplets in the fundamental representation and $N_{\bar{f}}$ hypermultiplets in the anti-fundamental representation, the Borel summability of each instanton sector is proven by combining the methods of [73] and [72].

\subsection{Extension to $S^{1} \times C_{g} \times \Sigma_{h}$}

As our final consideration of the general features of cohomological localization in five dimensions, we discuss how the prescriptions of this section should be adapted when the gauge theory is put on the five-manifold $M_{5}=S^{1} \times C_{g} \times \Sigma_{h}$; this is the five-dimensional lift of the discussion in section 3.6. The direct product $S^{1} \times C_{g} \times \Sigma_{h}$ is a special case, which is not included in our previous discussion for two reasons. Firstly, although it clearly corresponds to a regular fibration, this geometry does not follow directly from a rigid supergravity 
background; instead, we have to first put the theory on $\mathbb{R} \times C_{g} \times \Sigma_{h}$ using a supergravity background and then compactify. The second difference from the other U(1) fibrations discussed in this section is the localization locus. Supersymmetric localization on $S^{1} \times S^{4}$ was studied in [74-76]. The $Q_{\varepsilon}$-exact action of [75, eq. (4.12)] in our notation is proportional to

$$
\int_{M_{5}}\left(\frac{1}{2}(F \wedge * F)-(\mathrm{D} \sigma \hat{\wedge} * \mathrm{D} \sigma)+\kappa \wedge\left(-(\sigma, \sigma) \omega_{C_{g}} \wedge \omega_{\Sigma_{h}}+\frac{1}{2}(\sigma, F) \wedge \omega_{C_{g}}-\frac{1}{4}(F \wedge F)\right)\right)
$$

where we dropped the contributions from fields which must be set to zero at the localization locus. Bearing in mind that we will have to rotate $\sigma \mapsto \mathrm{i} \sigma_{0}$ and integrate over $\sigma_{0} \in \mathfrak{g}$, the saddle points of this action require $\sigma$ to be covariantly constant and $F$ to vanish in the direction of $S^{1}$. This implies that the gauge connection has a constant component $a_{0}$ along $S^{1}$, and we can gauge rotate $a_{0}$ into a Cartan subalgebra of $\mathfrak{g}$. Moreover, the curvature

$$
F_{C_{g} \times \Sigma_{h}}=f_{C_{g}} \omega_{C_{g}}+f_{\Sigma_{h}} \omega_{\Sigma_{h}}
$$

is a saddle point if $f_{C_{g}}=2 \sigma=f_{\Sigma_{h}}$, subject to the additional condition $\left[a_{0}, f_{C_{g}}\right]=0$. This vanishing Lie bracket guarantees that $\sigma$ is constant and can be conjugated into the same Cartan subalgebra as $a_{0}$. Therefore the full localization locus inside the Coulomb branch is parametrized by $a_{0}$ and $\vec{m} \in \Lambda$, an element of the co-root lattice in the same Cartan subalgebra as $a_{0}$, through

$$
A=a_{0} \kappa+\vec{m}\left(A_{C_{g}}+A_{\Sigma_{h}}\right) \quad \text { and } \quad \sigma=\frac{\vec{m}}{r}
$$

where $A_{C_{g}}$ and $A_{\Sigma_{h}}$ are monopole connections on $C_{g}$ and $\Sigma_{h}$, respectively, satisfying

$$
\int_{C_{g}} \mathrm{~d} A_{C_{g}}=\frac{1}{2} \operatorname{vol}\left(C_{g}\right) \quad \text { and } \quad \int_{\Sigma_{h}} \mathrm{~d} A_{\Sigma_{h}}=\frac{1}{2} \operatorname{vol}\left(\Sigma_{h}\right) .
$$

The pushdown to four dimensions in the present geometry is the usual Kaluza-Klein dimensional reduction, in contrast to the reduction of section 4.6.

The triviality of the $\mathrm{U}(1)$ fibration in the present case implies that the index of the twisted Dolbeault complex is given by

$$
\text { index } \bar{\partial}^{(m)}=(1-g)(1-h) \text {. }
$$

With the same notation $q=\mathrm{e}^{-\frac{\epsilon}{r}}$ from section 3.6, we immediately find

$$
\begin{aligned}
Z_{\mathrm{vec}}\left(S^{1} \times C_{g} \times \Sigma_{h}\right) & =Z_{\mathrm{vec}}\left(S^{1} \times C_{g}\right)^{1-h} \\
& =\prod_{\alpha \in \triangle} q^{-\frac{1}{2}(1-g)(1-h)|(\alpha, \vec{m})|}\left(1-\mathrm{e}^{-\mathrm{i} \epsilon\left(\alpha, a_{0}\right)} q^{|(\alpha, \vec{m})|}\right)^{(1-g)(1-h)} .
\end{aligned}
$$

This lifts the result of section 3.6 to the five-dimensional manifold $M_{5}=S^{1} \times C_{g} \times \Sigma_{h} \cdot{ }^{12}$

\footnotetext{
${ }^{12}$ As in section 3.6, we omit a factor $\prod_{\alpha \in \Delta}\left(\alpha, a_{0}\right)^{-1}$, which cancels the Jacobian arising from the integration over $a_{0}$ after gauge rotation into the chosen Cartan subalgebra.
} 


\section{$\mathcal{N}=2$ perturbative partition functions on $T^{3} \times \Sigma_{h}$}

View the three-torus $T^{3}$ as the trivial circle bundle over $C_{1}=T^{2}$. It follows from (4.64) that in this case the index of the Dolbeault complex is zero, and so from the localization formula (4.32) with $A^{(0)}=0$ together with (4.34) we obtain

$$
Z_{\mathrm{vec}}^{\text {pert }}\left(T^{3} \times \Sigma_{h}\right)=\prod_{\alpha \in \triangle_{+}}\left(\alpha, \sigma_{0}\right)^{-2} \quad \text { and } \quad Z_{\text {hyp }}^{\text {pert }}\left(T^{3} \times \Sigma_{h}\right)=1 .
$$

For the supersymmetric Yang-Mills theory on $T^{3} \times \Sigma_{h}$, the only non-trivial contributions to the one-loop determinants come from the ghosts in the vector multiplet, which produce a factor that exactly cancels the Jacobian arising from conjugation of $\sigma_{0}$ into the Cartan subalgebra $\mathfrak{t}$, as mentioned before. Hence the perturbative partition function is given by a Gaussian integral

$$
Z_{\mathcal{N}=2}^{\text {pert }}\left(T^{3} \times \Sigma_{h}\right)=\int_{\mathfrak{t}_{\text {reg }}} \mathrm{d} \tilde{\sigma} \mathrm{e}^{-\frac{4 \pi^{3} \mathrm{vol}\left(\Sigma_{h}\right)}{r g_{\mathrm{YM}}^{2}}(\tilde{\sigma}, \tilde{\sigma})} .
$$

For a quiver gauge theory with unitary gauge group $G=\mathrm{U}\left(N_{1}\right) \times \mathrm{U}\left(N_{2}\right) \times \cdots \times \mathrm{U}\left(N_{n}\right)$, this reads

$$
Z_{\mathcal{N}=2}^{\text {pert }}\left(T^{3} \times \Sigma_{h}\right)=\left(\frac{r g_{\mathrm{YM}}^{2}}{4 \pi^{3} \operatorname{vol}\left(\Sigma_{h}\right)}\right)^{\frac{1}{2}\left(N_{1}+\cdots+N_{n}\right)} .
$$

Note that the perturbative partition function on $T^{3} \times \Sigma_{h}$ does not distinguish between a non-abelian theory with gauge group $G=\mathrm{U}(N)$ and an abelian quiver theory with gauge group $G=\mathrm{U}(1)^{N}$.

\section{$5 \quad q$-deformed Yang-Mills theories from cohomological localization}

This final section is devoted to the study of five-dimensional supersymmetric Yang-Mills theory on $S_{b}^{3} \times \Sigma_{h}$, where $S_{b}^{3}$ is either the squashed sphere of [29] or the ellipsoid of [28] (see appendix B for details); recall from section 2.1 that these are the five-dimensional theories that naturally descend from six-dimensional superconformal field theories on squashed geometries. We show how $q$-deformations of Yang-Mills theory on a Riemann surface $\Sigma_{h}$ arise from our localization procedure. Our formula for the partition function of the standard $q$-deformed Yang-Mills theory in section 5.1 improves the result of [10, 15], wherein the Gaussian term was not retained; as we discuss, this Boltzmann factor is important for applications to holography. Both treatments of [10] and [15] focus on the zero area limit where $\operatorname{vol}\left(\Sigma_{h}\right) \rightarrow 0$, which hides the fact that the resulting $q$-deformed Yang-Mills theory has $p=1$. We elucidate the geometric significance of this new $q$-deformation through an analysis of the resulting matrix model on the sphere $\Sigma_{0}=S^{2}$, by adapting the procedure of [34] to the present case.

\subsection{Localization on $S^{3} \times \Sigma_{h}$}

Consider the $\mathcal{N}=1$ partition function without matter on $S^{3} \times \Sigma_{h}$, beyond the perturbative calculation of section 4.5. More precisely, we include the full set of solutions obtained 
in section 4.6 , but discard the four-dimensional point-like instantons. ${ }^{13}$ The resulting partition function is given by

$$
Z_{\mathcal{N}=1}\left(S^{3} \times \Sigma_{h}\right)=\sum_{A^{(0)}} \int_{\mathfrak{t}_{\mathrm{reg}}} \mathrm{d} \sigma_{0} \mathrm{e}^{-S_{\mathrm{cl}}\left(F, \sigma_{0}\right)} \prod_{\alpha \in \triangle_{+}} \sinh (\pi r(\alpha, \phi))^{2-2 h},
$$

with $S_{\mathrm{cl}}$ the action evaluated at the localization locus:

$$
S_{\mathrm{cl}}\left(F, \sigma_{0}\right)=S_{\mathrm{YM}}(F)+\frac{1}{2 g_{\mathrm{YM}}^{2}} \int_{S^{3} \times \Sigma_{h}}\left(\frac{1}{r^{2}}\left(\sigma_{0}, \sigma_{0}\right) \kappa \wedge \mathrm{d} \kappa \wedge \omega_{\Sigma_{h}}+\frac{1}{r}\left(\sigma_{0}, F\right) \wedge \kappa \wedge \mathrm{d} \kappa\right)
$$

The first summand in the action, $S_{\mathrm{YM}}(F)$, is the five-dimensional Yang-Mills action evaluated in section 4.6 :

$$
\begin{aligned}
S_{\mathrm{YM}}(F) & =\frac{1}{2 g_{\mathrm{YM}}^{2}} \int_{S^{3} \times \Sigma_{h}} \kappa \wedge\left(F_{H,+} \wedge F_{H,+}\right) \\
& =-\frac{\pi r}{g_{\mathrm{YM}}^{2}} \int_{S^{2} \times \Sigma_{h}}\left(\left.\left.F\right|_{S^{2} \times \Sigma_{h}} \wedge F\right|_{S^{2} \times \Sigma_{h}}\right) \\
& =\frac{8 \pi^{3} r}{g_{\mathrm{YM}}^{2}}(\vec{m}, \vec{n}),
\end{aligned}
$$

where in the second equality we used the defining equations, and in the last equality $(\vec{m}, \vec{n}) \in \mathbb{Z}$ is proportional to the second Chern character $\mathrm{ch}_{2}$ associated to the pertinent principal $G$-bundle over $S^{2} \times \Sigma_{h}$.

The sum in the partition function runs over the $G$-connections $A^{(0)}$ whose curvature $F$ satisfies the fixed point equation

$$
v\llcorner * F=F .
$$

These solutions were studied in section 4.6. It is straightforward to check that connections whose curvature lives on $\Sigma_{h}$, that is $F=\left.F\right|_{\Sigma_{h}}$, do not satisfy this equation. In other words, connections that are flat when projected onto $S^{3}$, and which thus belong to the localization locus of a purely three-dimensional theory, do not belong to the localization locus of the fully twisted five-dimensional theory. The flat connection on $S^{3}$ does belong to the localization locus of the five-dimensional theory which is partially twisted along $\Sigma_{h}$ (with arbitrary $\left.A\right|_{\Sigma_{h}}$ ), but not to the localization locus of the fully twisted theory; the partition function of the field theory topologically twisted along $\Sigma_{h}$ is important from the perspective of the six-dimensional theory and its reduction to four-dimensional theories of class $\mathcal{S}[10,15,16]$. The same argument can be made for connections which are flat along $\Sigma_{h}$, which then only belong to the localization locus of the five-dimensional theory which is partially twisted along $S^{3}$ (with arbitrary $\left.A\right|_{S^{3}}$ ) $[36,37,39]$.

\footnotetext{
${ }^{13}$ This sector of the partition function was named "perturbative" in [16]. However, in the present paper the term perturbative refers to the expansion around the trivial connection, while the subsector of the full partition function we are considering now includes a much wider class of solutions. A proper definition hinted at in [16, section 2.4] might be "partition function neglecting codimension four field configurations". These are non-perturbative with respect to an expansion in the geometric area parameter $\operatorname{vol}\left(\Sigma_{h}\right)$.
} 
We can write

$$
Z_{\mathcal{N}=1}\left(S^{3} \times \Sigma_{h}\right)=\sum_{\vec{m}, \vec{n} \in \mathbb{Z}^{\operatorname{rank}(G)}} \int_{\mathfrak{t}_{\mathrm{reg}}} \mathrm{d} \phi \mathrm{e}^{-S_{\mathrm{cl}}(\vec{m}, \vec{n} ; \phi)} \prod_{\alpha \in \triangle_{+}} \sinh (\pi r(\alpha, \phi))^{2-2 h} .
$$

We shifted the integration variable to $\phi=-\sigma_{0}-v\left\llcorner A^{(0)}\right.$. This is the natural variable to use when descending to a four-dimensional description, as the sum in (5.5) is taken over the gauge fluxes $\vec{m}$ and $\vec{n}$ through $S^{2}$ and $\Sigma_{h}$, respectively; the first and second Chern characters associated to the principal $G$-bundle over $S^{2} \times \Sigma_{h}$ are obtained by using the pairing $(\cdot, \cdot)$. The action evaluated at the fixed points consists of three terms:

$S_{\mathrm{cl}}(\vec{m}, \vec{n} ; \phi)=S_{\mathrm{YM}}(\vec{m}, \vec{n})+\frac{1}{2 g_{\mathrm{YM}}^{2}} \int_{S^{3} \times \Sigma_{h}}\left(\frac{1}{r^{2}}(\phi, \phi) \kappa \wedge \mathrm{d} \kappa \wedge \omega_{\Sigma_{h}}-\frac{1}{r}\left(\phi,\left.F\right|_{S^{2} \times \Sigma_{h}}\right) \wedge \kappa \wedge \mathrm{d} \kappa\right)$,

where the first summand is written in (5.3). Passing to the scaled variable $\tilde{\phi}=r \phi$, we get

$$
\begin{aligned}
S_{\mathrm{cl}}(\vec{m}, \vec{n} ; \tilde{\phi})= & S_{\mathrm{YM}}(\vec{m}, \vec{n}) \\
& +\frac{1}{g_{\mathrm{YM}}^{2}}\left(\frac{8 \pi^{2} \operatorname{vol}\left(\Sigma_{h}\right)}{r}(\tilde{\phi}, \tilde{\phi})+\frac{2 \pi \mathrm{i}}{r^{2}} \int_{S^{3} \times \Sigma_{h}} \frac{\mathrm{i}}{4 \pi}\left(\tilde{\phi},\left.F\right|_{S^{2} \times \Sigma_{h}}\right) \wedge \kappa \wedge \mathrm{d} \kappa\right) .
\end{aligned}
$$

Zero flux sector. We will now restrict ourselves to those connections for which $S_{\mathrm{YM}}(\vec{m}, \vec{n})=0$. Contributions with non-vanishing second Chern character are exponentially suppressed. We turn off the gauge fluxes through $S^{2}, \vec{m}=\overrightarrow{0}$, and follow standard techniques from two-dimensional Yang-Mills theory, see in particular [52]. Using

$$
\frac{1}{2} \int_{S^{3} \times \Sigma_{h}} \frac{\mathrm{i}}{2 \pi}\left(\tilde{\phi},\left.F\right|_{S^{2} \times \Sigma_{h}}\right) \wedge \kappa \wedge \mathrm{d} \kappa=4 \pi^{2} r^{3}(\tilde{\phi}, \vec{n})
$$

and summing over $\vec{n} \in \mathbb{Z}^{\operatorname{rank}(G)}$, the third summand in the action (5.7) produces the delta-function constraint

$$
\frac{4 \pi^{2} r}{g_{\mathrm{YM}}^{2}} \tilde{\phi}=\vec{k}
$$

for some integer vector $\vec{k} \in \mathbb{Z}^{\operatorname{rank}(G)}$ which can be identified with a regular weight of $\mathfrak{g}$. Plugging this into the remaining Gaussian part of the action gives

$$
\frac{g_{\mathrm{YM}}^{2}}{4 \pi r} \frac{\operatorname{vol}\left(\Sigma_{h}\right)}{\pi r^{2}}(\vec{k}, \vec{k}) \text {. }
$$

In the one-loop determinant, we obtain

$$
\sinh (\pi r(\alpha, \phi))=\sinh (\pi(\alpha, \tilde{\phi}))=\sinh \left(\frac{g_{\mathrm{YM}}^{2}}{4 \pi r}(\alpha, \vec{k})\right)=-[(\alpha, \vec{k})]_{q}
$$

where

$$
q=\mathrm{e}^{-g_{\mathrm{str}}} \quad \text { with } \quad g_{\mathrm{str}}:=\frac{g_{\mathrm{YM}}^{2}}{2 \pi r} .
$$

The final form of the partition function is then

$$
Z_{\mathcal{N}=1}^{\vec{m}=\overrightarrow{0}}\left(S^{3} \times \Sigma_{h}\right)=\sum_{\vec{k} \in\left(\mathbb{Z}^{\operatorname{rank}(G)}\right)_{\operatorname{reg}}} \prod_{\alpha \in \triangle_{+}}[(\alpha, \vec{k})]_{q}^{2-2 h} q^{\frac{1}{2}(\vec{k}, \vec{k}) \frac{\operatorname{vol}\left(\Sigma_{h}\right)}{\pi r^{2}}} .
$$


From (5.12) we identify the six-dimensional radius $\beta=\frac{g_{\mathrm{YM}}^{2}}{2 \pi}$, and it is instructive to rewrite

$$
q^{\frac{1}{2}(\vec{k}, \vec{k}) \frac{\operatorname{vol}\left(\Sigma_{h}\right)}{\pi r^{2}}}=\exp \left(-(\vec{k}, \vec{k}) \frac{2 \pi \beta \operatorname{vol}\left(\Sigma_{h}\right)}{\operatorname{vol}_{\kappa}\left(S^{3}\right)}\right),
$$

where $\operatorname{vol}_{\kappa}\left(S^{3}\right)$ is the volume of $S^{3}$ taken with respect to the contact structure $\kappa$, in our normalization.

The expression (5.13) is the partition function of the standard $q$-deformed twodimensional Yang-Mills theory on $\Sigma_{h}$ (cf. section 2.2), at $p=1$ and with Gaussian weight corrected by the ratio of volumes between the fibre Riemann surface $\Sigma_{h}$ and the total space sphere $S^{3}$. This ratio of volumes matches exactly with [31]. For unitary gauge group $G=\mathrm{U}(N)$, this is reminiscent of the large $N$ free energy of gauge theories with a holographic dual. In particular, for $\Sigma_{h}=\Sigma_{0}=S^{2}$, the $N^{3}$ behaviour of the free energy at large $N$ in the present theory follows immediately from the large $N$ limit of $q$-deformed YangMills theory, which in turn is given by the free energy of Chern-Simons theory on $S^{3}$ as we are in the case $p=1$. This indeed gives the right answer for a gauge theory on a SasakiEinstein five-manifold which has a holographic dual [77]. See [78] for a discussion and a similar example where the $N^{3}$ behaviour of the free energy in five dimensions is extracted from Chern-Simons gauge theory in three dimensions. See also [79] for the localization of five-dimensional maximally supersymmetric Yang-Mills theory to a three-dimensional subsector, and the relation with Chern-Simons theory.

We notice that the string coupling $g_{\text {str }}$ depends only on the ratio $\frac{g_{\mathrm{YM}}^{2}}{r}$. This is consistent with dimensional reduction from the $(2,0)$ theory on $S^{1} \times S^{3} \times \Sigma_{h}$ discussed in section 2.1. In the six-dimensional setting, $g_{\mathrm{YM}}^{2}$ plays the role of the circumference of the circle $S^{1}$ on which we have reduced. On the other hand, the small area limit $\operatorname{vol}\left(\Sigma_{h}\right) \rightarrow 0$ gives the superconformal index of the four-dimensional gauge theory on $S^{1} \times S^{3}$, consistently with the conjecture of [30]. From (5.14) it is evident that the small vol $\left(\Sigma_{h}\right)$ limit is the same as the large $\operatorname{vol}_{\kappa}\left(S^{3}\right)$ limit which decompactifies the three-sphere $S^{3}$ to $\mathbb{R}^{3}$.

Reinstating gauge fluxes. We have so far restricted ourselves to the sector where $S_{\mathrm{YM}}(\vec{m}, \vec{n})=0$. In general we have to consider additional solutions which are given by connections whose curvature lives on $S^{2} \times \Sigma_{h}$ with non-trivial second Chern character $\operatorname{ch}_{2} \neq 0$, and

$$
S_{\mathrm{YM}}(\vec{m}, \vec{n})=\frac{8 \pi^{3} r}{g_{\mathrm{YM}}^{2}}(\vec{m}, \vec{n})=2 \pi \mathrm{i}\left(-\mathrm{i} \frac{4 \pi^{2} r}{g_{\mathrm{YM}}^{2}}(\vec{m}, \vec{n})\right),
$$

where as above $\vec{n}$ is the gauge flux through $\Sigma_{h}$ and $\vec{m}$ is the gauge flux through $S^{2}$. The full partition function includes this term in the action, along with a sum over $\vec{m} \in \mathbb{Z}^{\operatorname{rank}(G)}$. The bracketed term has exactly the same coefficient as a BF-type term in the action. This means that the procedure we used for the $\vec{m}=\overrightarrow{0}$ sector should be modified by a shift $\tilde{\phi} \mapsto \tilde{\phi}-\mathrm{i} \vec{m}$ after the sum over all $\vec{n} \in \mathbb{Z}^{\operatorname{rank}(G)}$. We finally arrive at

$$
\begin{gathered}
Z_{\mathcal{N}=1}\left(S^{3} \times \Sigma_{h}\right)=\sum_{\vec{m} \in \mathbb{Z}^{\operatorname{rank}(G)}} \sum_{\vec{k} \in\left(\mathbb{Z}^{\operatorname{rank}(G)}\right)_{\operatorname{reg}}} \prod_{\alpha \in \triangle_{+}}\left([(\alpha, \vec{k}-\mathrm{i} \vec{m})]_{q}[(\alpha, \vec{k}+\mathrm{i} \vec{m})]_{q}\right)^{1-h} \\
\times q^{\frac{1}{2}(\vec{k}-\mathrm{i} \vec{m}, \vec{k}-\mathrm{i} \vec{m}) \frac{\operatorname{vol}\left(\Sigma_{h}\right)}{\pi r^{2}}} .
\end{gathered}
$$

The $q$-deformed measure has in fact precisely the right form to support non-trivial fluxes $\vec{m}$. 


\subsection{Localization on squashed $S_{b}^{3} \times \Sigma_{h}$}

There exist two types of squashings of $S^{3}$ that can be lifted to five dimensions with $\mathcal{N}=2$ supersymmetry. The first type is the familiar case of [28]. From the point of view of cohomological localization, the squashing simply corresponds to a rescaling of the fibre radius, and the results for the one-loop determinants on $S_{b}^{3} \times \Sigma_{h}$ are exactly the same as for the round sphere $S^{3}$, but with fibre radius $\epsilon \neq r$, where $r$ is the radius of the base $S^{2}$. See appendix B for further details.

The other squashed sphere, which is a regular fibration, is that of [29]. We did not give a formal derivation of the one-loop determinants in the cohomological gauge theory for this background. However, we know that the Killing vector field $v$ has closed orbits and is parallel to the Reeb vector field $\xi$. We can therefore lift the results from $S_{b}^{3}$ to $S_{b}^{3} \times \Sigma_{h}$, and the one-loop determinants are given by

$$
\begin{aligned}
& Z_{\mathrm{vec}}\left(S_{b}^{3} \times \Sigma_{h}\right)=\left(\prod_{\alpha \in \triangle_{+}} \frac{\sinh \left(\pi \epsilon_{1}\left(\alpha, \sigma_{0}\right)\right) \sinh \left(\pi \epsilon_{2}\left(\alpha, \sigma_{0}\right)\right)}{\pi^{2} \epsilon_{1} \epsilon_{2}\left(\alpha, \sigma_{0}\right)^{2}}\right)^{1-h}, \\
& Z_{\mathrm{hyp}}\left(S_{b}^{3} \times \Sigma_{h}\right)=\left(\prod_{\rho \in \Lambda_{R}} \mathrm{~s}_{b}\left(\frac{\mathrm{i}}{2}\left(b+b^{-1}\right)(1-\Delta)-r\left(\rho, \sigma_{0}\right)\right)\right)^{1-h},
\end{aligned}
$$

which agrees with $[16$, section 2]. We adopted the notation

$$
\epsilon_{1}=r b \quad \text { and } \quad \epsilon_{2}=r b^{-1} \quad \text { with } \quad b=\frac{1-\mathrm{i} u}{\sqrt{1+u^{2}}}
$$

from appendix B.

The computation of the full $\mathcal{N}=1$ partition function without matter on $S_{b}^{3} \times \Sigma_{h}$ proceeds exactly as in section 5.1, with only a modification in the one-loop determinant. This results in a bi-orthogonalization of the $q$-deformed measure, and the partition function in the sector of vanishing second Chern character $\mathrm{ch}_{2}=0$ is given by

$$
Z_{\mathcal{N}=1}^{\overrightarrow{\mathcal{N}}=\overrightarrow{0}}\left(S_{b}^{3} \times \Sigma_{h}\right)=\sum_{\vec{k} \in\left(\mathbb{Z}^{\mathrm{rank}(G)}\right)_{\mathrm{reg}}} \prod_{\alpha \in \triangle_{+}}\left([b(\alpha, \vec{k})]_{q}\left[b^{-1}(\alpha, \vec{k})\right]_{q}\right)^{1-h} q^{\frac{1}{2}(\vec{k}, \vec{k}) \frac{\mathrm{vol}\left(\Sigma_{h}\right)}{\pi r^{2}}} .
$$

The extension to the full partition function including non-trivial gauge fluxes $\vec{m}$ through the base $S^{2}$ is exactly as described in section 5.1, as we are presently working in the regular case. After inclusion of gauge fluxes $\vec{m} \neq \overrightarrow{0}$, our result appears to be only in partial agreement with [16,35], where the field theory is first defined on $S_{b}^{3} \times \mathbb{R}^{2}$, then dimensionally reduced, and finally put on the Riemann surface $\Sigma_{h}$ with a twist. That procedure allows for additional background fluxes for the flavour symmetry, which do not appear in our framework nor in [10].

\subsection{Localization on ellipsoid $S_{b}^{3} \times \Sigma_{h}$}

We now consider the geometry $S_{b}^{3} \times \Sigma_{h}$ for the ellipsoid $S_{b}^{3}$ of [28]. Most of the steps are the same as in the round $S^{3}$ case of section 5.1. The action at the localization locus is 
again given by

$$
S_{\mathrm{cl}}\left(F, \sigma_{0}\right)=S_{\mathrm{YM}}(F)+\frac{1}{2 g_{\mathrm{YM}}^{2}} \int_{S^{3} \times \Sigma_{h}}\left(\frac{1}{r^{2}}\left(\sigma_{0}, \sigma_{0}\right) \kappa \wedge \mathrm{d} \kappa \wedge \omega_{\Sigma_{h}}+\frac{1}{r}\left(\sigma_{0}, F\right) \wedge \kappa \wedge \mathrm{d} \kappa\right),
$$

with

$$
S_{\mathrm{YM}}(F)=\frac{1}{2 g_{\mathrm{YM}}^{2}} \int_{S^{3} \times \Sigma_{h}}(F \wedge * F) .
$$

The zero flux sector of the partition function is

$$
\begin{aligned}
Z_{\mathcal{N}=1}^{\vec{m}=\overrightarrow{0}}\left(S_{b}^{3} \times \Sigma_{h}\right)= & \sum_{\vec{n} \in \mathbb{Z}^{\operatorname{rank}(G)}} \int_{\mathrm{t}_{\mathrm{reg}}} \mathrm{d} \sigma_{0} \mathrm{e}^{-S_{\mathrm{cl}}\left(\overrightarrow{0}, \vec{n} ; \sigma_{0}\right)} \\
& \times \prod_{\alpha \in \triangle_{+}}\left(\sinh \left(\pi r b\left(\alpha, \sigma_{0}\right)\right) \sinh \left(\pi r b^{-1}\left(\alpha, \sigma_{0}\right)\right)\right)^{1-h} .
\end{aligned}
$$

Changing variable $\tilde{\sigma}=r \sigma_{0}$ and repeating the same steps used in section 5.2, we arrive at

$$
\begin{aligned}
Z_{\mathcal{N}=1}^{\vec{m}=\overrightarrow{0}}\left(S_{b}^{3} \times \Sigma_{h}\right)= & \sum_{\vec{k} \in\left(\mathbb{Z}_{\operatorname{rank}(G)}\right)_{\operatorname{reg}}} \mathrm{e}^{-\frac{g_{\mathrm{str}}}{2}(\vec{k}, \vec{k}) \frac{\mathrm{vol}\left(\Sigma_{h}\right)}{\pi r^{2}}} \\
& \times \prod_{\alpha \in \triangle_{+}}\left(\sinh \left(b \frac{g_{\mathrm{str}}(\alpha, \vec{k})}{2}\right) \sinh \left(b^{-1} \frac{g_{\mathrm{str}}(\alpha, \vec{k})}{2}\right)\right)^{1-h}
\end{aligned}
$$

where as before we defined the string coupling $g_{\mathrm{str}}=\frac{g_{\mathrm{YM}}^{2}}{2 \pi r}=\frac{\beta}{r}$. The final expression can be rewritten in terms of $q=\mathrm{e}^{-g_{\text {str }}}$ as

$$
Z_{\mathcal{N}=1}^{\vec{m}=\overrightarrow{0}}\left(S_{b}^{3} \times \Sigma_{h}\right)=\sum_{\vec{k} \in\left(\mathbb{Z}^{\operatorname{rank}(G)}\right)_{\operatorname{reg}}} q^{\frac{1}{2}(\vec{k}, \vec{k}) \frac{\operatorname{vol}\left(\Sigma_{h}\right)}{\pi r^{2}}} \prod_{\alpha \in \triangle_{+}}\left([b(\alpha, \vec{k})]_{q}\left[b^{-1}(\alpha, \vec{k})\right]_{q}\right)^{1-h} .
$$

From (5.22) we see that the perturbative partition function on $S_{b}^{3} \times S^{2}$, retaining only the contribution from the trivial flat connection $A^{(0)}=0$, coincides with the perturbative partition function of Chern-Simons gauge theory on the lens space $L(p, 1)$, continued to arbitrary values $p=b^{2} \in \mathbb{R}$ :

$$
Z_{\mathcal{N}=1}^{\text {pert }}\left(S_{b}^{3} \times S^{2}\right)=\int_{\mathfrak{t}_{\text {reg }}} \mathrm{d} \sigma \mathrm{e}^{-\frac{1}{2 g_{\mathrm{str}}}(\sigma, \sigma) \frac{\operatorname{vol}\left(\Sigma_{h}\right)}{\pi r^{2}}} \prod_{\alpha \in \triangle_{+}} \sinh \left(b \frac{(\alpha, \sigma)}{2}\right) \sinh \left(b^{-1} \frac{(\alpha, \sigma)}{2}\right),
$$

where we rescaled $\sigma=g_{\text {str }} \sigma_{0}$. This becomes more evident if we use instead an asymmetric length scaling to define the variable $\tilde{\sigma}=\epsilon_{1} \sigma_{0}=r b \sigma_{0}$. For general genus $h$ we then arrive at the discrete matrix model

$$
Z_{\mathcal{N}=1}^{\vec{m}=\overrightarrow{0}}\left(S_{b}^{3} \times \Sigma_{h}\right)=\frac{1}{b^{\operatorname{rank}(G)}} \sum_{\vec{k} \in\left(\mathbb{Z}^{\operatorname{rank}(G)}\right)_{\operatorname{reg}}} q^{\frac{1}{2}(\vec{k}, \vec{k}) \frac{\operatorname{vol}\left(\Sigma_{h}\right)}{\pi r^{2}}} \prod_{\alpha \in \triangle_{+}}\left([(\alpha, \vec{k})]_{q}\left[b^{-2}(\alpha, \vec{k})\right]_{q}\right)^{1-h} .
$$

Further details and analysis of this matrix model for genus $h=0$ and gauge group $G=$ $\mathrm{U}(N)$, along the lines of [34], are provided in section 5.4 below. 
Reinstating the additional contributions of contact instantons, with non-vanishing fluxes through the base $S^{2}$ of the U(1) fibration of $S_{b}^{3}$, is a much more subtle issue. This is because the $\mathrm{U}(1)$-action now involves the Killing vector field $v$ which differs from the Reeb vector field $\xi$ on $S_{b}^{3}$, so that contractions with $v$ do not separate the horizontal and vertical parts of the differential forms involved.

\subsection{The matrix model}

We focus now on the partition function $Z_{\mathcal{N}=1}^{\vec{m}=0}\left(S_{b}^{3} \times S^{2}\right)$ for gauge group $G=\mathrm{U}(N)$. The partition function is formally the same for either the squashed sphere or the ellipsoid $S_{b}^{3}$. Only the geometric meaning of the squashing parameter $b$ is different in the two cases, in particular $b$ is a complex number of unit modulus $|b|=1$ for the squashed sphere and $b>0$ is real for the ellipsoid. However, as the partition function can be analytically continued in both cases, there is no difference in practice.

Our goal is then to study the discrete random matrix ensemble with partition function

$$
\begin{aligned}
\mathcal{Z}_{N}(b)= & \frac{1}{b^{N} N !} \sum_{\vec{\ell} \in \mathbb{Z}^{N}} \mathrm{e}^{-\frac{g_{\mathrm{str}}}{2} \sum_{j=1}^{N} \ell_{j}^{2}} \\
& \times \prod_{1 \leq j<k \leq N} 4 \sinh \left(\frac{g_{\mathrm{str}}}{2}\left(\ell_{j}-\ell_{k}\right)\right) \sinh \left(\frac{g_{\mathrm{str}}}{2 b^{2}}\left(\ell_{j}-\ell_{k}\right)\right),
\end{aligned}
$$

which can be identified with the discrete version of the bi-orthogonal Stieltjes-Wigert ensemble studied in [80]. If $p:=b^{2} \in \mathbb{Z}$, the continuous version of the bi-orthogonal StieltjesWigert ensemble provides the partition function of Chern-Simons theory on the lens space $L(p, 1)$. In the limit $b \rightarrow 1$ we recover the partition function of $q$-deformed Yang-Mills theory constructed from the monopole bundle over $S^{2}$ with $p=1$, whose continuous counterpart is Chern-Simon theory on $S^{3}$ (with analytically continued level). In the present setting, $b^{2}$ may be any positive real number, not necessarily integer, and indeed the analysis of the bi-orthogonal Stieltjes-Wigert ensemble in [80] does not rely on $p$ being integer. We shall now clarify the geometric significance of the dependence on the squashing parameter $b$ of this $q$-deformed Yang-Mills theory.

Semi-classical expansion. Following [34], we will begin by performing a modular inversion of the series (5.27) to obtain the dual description of the $q$-deformed Yang-Mills matrix model in terms of instanton degrees of freedom. For this, we consider the function

$$
F_{b}\left(x_{1}, \ldots, x_{N}\right)=\mathrm{e}^{-\frac{g_{\mathrm{str}}}{4} \sum_{j=1}^{N} x_{j}^{2}} \prod_{1 \leq j<k \leq N} 2 \sinh \left(\frac{g_{\mathrm{str}}}{2 b^{2}}\left(x_{j}-x_{k}\right)\right)
$$

of continuous variables $\left(x_{1}, \ldots, x_{N}\right) \in \mathbb{R}^{N}$. Its Fourier transform is given by

$$
\begin{aligned}
\widehat{F}_{b}\left(y_{1}, \ldots, y_{N}\right):= & \int_{\mathbb{R}^{N}} \mathrm{~d} x \mathrm{e}^{\sum_{j=1}^{N}\left(2 \pi \mathrm{i} x_{j} y_{j}-\frac{g_{\text {str }}}{4} x_{j}^{2}\right)} \prod_{1 \leq j<k \leq N}\left(\mathrm{e}^{\frac{\left(x_{j}-x_{k}\right) g_{\text {str }}}{2 b^{2}}}-\mathrm{e}^{-\frac{\left(x_{j}-x_{k}\right) g_{\text {str }}}{2 b^{2}}}\right) \\
= & \mathrm{e}^{-\frac{4 \pi^{2}}{g_{\text {str }}} \sum_{j=1}^{N}\left(y_{j}+\frac{\mathrm{i}(N-1) g_{\text {str }}}{4 \pi b^{2}}\right)^{2}+\frac{N(N-1)^{2} g_{\text {str }}}{2 b^{4}}} \\
& \times \int_{\mathbb{R}^{N}} \mathrm{~d} u \mathrm{e}^{-\frac{g_{\text {str }}}{4} \sum_{j=1}^{N} u_{j}^{2}} \prod_{1 \leq j<k \leq N}\left(\mathrm{e}^{\frac{g_{\text {str }} u_{j}}{b^{2}}+\frac{4 \pi \mathrm{i} y_{j}}{b^{2}}}-\mathrm{e}^{\frac{g_{\text {str }} u_{k}}{b^{2}}+\frac{4 \pi \mathrm{i} y_{k}}{b^{2}}}\right),
\end{aligned}
$$


where we completed squares and changed integration variables to $u_{j}=x_{j}-\frac{4 \pi \mathrm{i}}{g_{\mathrm{str}}} y_{j}+$ $\frac{N-1}{b^{2}}$. We now change integration variables again, in the usual way for matrix models with hyperbolic interactions, by defining

$$
z_{j}=\mathrm{e}^{\frac{g_{\mathrm{str}} u_{j}}{b^{2}}+\frac{2 g_{\mathrm{str}}}{b^{4}}}
$$

to get

$$
\begin{aligned}
\widehat{F}_{b}\left(y_{1}, \ldots, y_{N}\right)= & \left(\frac{b^{2}}{g_{\text {str }}}\right)^{N} \mathrm{e}^{-\frac{g_{\text {str }}}{2 b^{4}} N\left(N^{2}+1\right)} \mathrm{e}^{-\frac{4 \pi^{2}}{g_{\text {str }}} \sum_{j=1}^{N}\left(y_{j}+\frac{\mathrm{i}(N-1) g_{\text {str }}}{4 \pi b^{2}}\right)^{2}} \\
& \times \int_{(0, \infty)^{N}} \mathrm{~d} z \mathrm{e}^{-\frac{b^{4}}{4 g_{\text {str }}} \sum_{j=1}^{N}\left(\log z_{j}\right)^{2}} \prod_{1 \leq j<k \leq N}\left(z_{j} \mathrm{e}^{\frac{4 \pi \mathrm{i} y_{j}}{b^{2}}}-z_{k} \mathrm{e}^{\frac{4 \pi \mathrm{i} y_{k}}{b^{2}}}\right) .
\end{aligned}
$$

The integral expression we have arrived at is exactly the same as in [34, eq. (3.14)] under the identification of the string coupling constant $\tilde{g}_{\mathrm{str}}$ there as $\tilde{g}_{\mathrm{str}}=\frac{g_{\mathrm{str}}}{b^{2}}$, which as we have seen is the coupling that reproduces the standard $q$-deformed Yang-Mills theory. We can therefore evaluate the integral using Stieltjes-Wigert polynomials to get

$$
\begin{aligned}
\widehat{F}_{b}\left(y_{1}, \ldots, y_{N}\right)= & \left(\frac{4 \pi}{g_{\text {str }}}\right)^{\frac{N}{2}} \mathrm{e}^{\frac{g_{\text {str }}}{6 b^{4}} N(N-1)(N-2)} \mathrm{e}^{-\frac{4 \pi^{2}}{g_{\text {str }}} \sum_{j=1}^{N}\left(y_{j}+\frac{\mathrm{i}(N-1) g_{\text {str }}}{4 \pi b^{2}}\right)^{2}} \\
& \times \prod_{1 \leq j<k \leq N}\left(\mathrm{e}^{\frac{4 \pi \mathrm{i} y_{j}}{b^{2}}}-\mathrm{e}^{\frac{4 \pi \mathrm{i} y_{k}}{b^{2}}}\right) .
\end{aligned}
$$

At this point, we apply the convolution theorem for Fourier transformations to get

$$
\begin{aligned}
Z_{b}\left(y_{1}, \ldots, y_{N}\right) & :=\int_{\mathbb{R}^{N}} \mathrm{~d} x \mathrm{e}^{2 \pi \mathrm{i} \sum_{j=1}^{N} x_{j} y_{j}} F_{b}\left(x_{1}, \ldots, x_{N}\right) F_{1}\left(x_{1}, \ldots, x_{N}\right) \\
& =\int_{\mathbb{R}^{N}} \mathrm{~d} t \widehat{F}_{b}\left(\frac{y_{1}-t_{1}}{2}, \ldots, \frac{y_{N}-t_{N}}{2}\right) \widehat{F}_{1}\left(\frac{y_{1}+t_{1}}{2}, \ldots, \frac{y_{N}+t_{N}}{2}\right) .
\end{aligned}
$$

After some calculation, we arrive finally at

$$
Z_{b}\left(y_{1}, \ldots, y_{N}\right)=\mathrm{e}^{-\frac{2 \pi^{2}}{g_{\mathrm{str}}} \sum_{j=1}^{N} y_{j}^{2}} \mathcal{W}_{b}\left(y_{1}, \ldots, y_{N}\right)
$$

with weight given by

$$
\begin{aligned}
\mathcal{W}_{b}\left(y_{1}, \ldots, y_{N}\right)= & \left(\frac{4 \pi}{g_{\mathrm{str}}}\right)^{N} \mathrm{e}^{\frac{g_{\mathrm{str}}}{12} N\left(N^{2}-1\right)\left(1+b^{-4}\right)} \int_{\mathbb{R}^{N}} \mathrm{~d} t \mathrm{e}^{-\frac{2 \pi^{2}}{g_{\mathrm{str}}} \sum_{j=1}^{N} t_{j}^{2}} \\
& \times \prod_{1 \leq j<k \leq N} 2\left(\cos \pi\left(y_{j k}\left(1+b^{-2}\right)+t_{j k}\left(1-b^{-2}\right)\right)\right. \\
& \left.\quad-\cos \pi\left(y_{j k}\left(1-b^{-2}\right)+t_{j k}\left(1+b^{-2}\right)\right)\right),
\end{aligned}
$$

where we adopted the shorthand notation $y_{j k}:=y_{j}-y_{k}$ and $t_{j k}:=t_{j}-t_{k}$. This correctly reproduces the weight of [34, eq. (3.20)] in the limit $b=1$.

The final step in developing the semi-classical expansion of the partition function (5.27) is Poisson resummation, and we finally arrive at

$$
\mathcal{Z}_{N}(b)=\frac{1}{b^{N} N !} \sum_{\vec{\ell} \in \mathbb{Z}^{N}} Z_{b}(\vec{\ell})=\frac{1}{b^{N} N !} \sum_{\vec{\ell} \in \mathbb{Z}^{N}} \mathrm{e}^{-\frac{2 \pi^{2}}{g_{\mathrm{str}}}(\vec{\ell}, \vec{\ell})} \mathcal{W}_{b}(\vec{\ell}) .
$$


This expression admits the standard interpretation as a sum over instanton solutions of the two-dimensional gauge theory: since the $q$-deformation arises here through one-loop determinants in the initial five-dimensional field theory, at the classical level this theory is just ordinary Yang-Mills theory on the sphere $S^{2}$. The exponential prefactors in the series (5.36) are then the classical contributions to the gauge theory path integral from the Yang-Mills action evaluated on instantons of topological charge $\ell_{j} \in \mathbb{Z}$ corresponding to a Dirac monopole of the $j$-th factor of the maximal torus $\mathrm{U}(1)^{N} \subset \mathrm{U}(N)$, while the integrals (5.35) are the fluctuation determinants around each instanton.

Rational limit and Chern-Simons theory on $\boldsymbol{L}(\boldsymbol{p}, s)$. Up to now the derivation of (5.36) worked for every positive real value of the squashing parameter $b$. Let us now specialise the squashing parameter to the rational values

$$
b^{2}=\frac{p}{s} \in \mathbb{Q}
$$

where $p$ and $s$ are coprime positive integers with $1 \leq s \leq p$. From the five-dimensional perspective that we started with, the ellipsoid Seifert manifold $S_{b}^{3}$ then has the topology of a lens space $L(p, s)$, viewed as a circle bundle over $S^{2}$ with two marked points [50]; the exceptional fibres over the marked points respectively makes them $\mathbb{Z}_{p}$ and $\mathbb{Z}_{s}$ orbifold points. The first Chern class of the line $\mathrm{V}$-bundle $\mathscr{L}(p, s)$ over the $\mathbb{P}^{1}$ orbifold associated to $L(p, s)$ is

$$
c_{1}(\mathscr{L}(p, s))=\frac{s}{p} \omega_{\mathbb{P}^{1}}
$$

which cancels the local curvatures at the marked points of $\mathbb{P}^{1}$ to ensure that the total degree of the Seifert fibration is zero. This is also homeomorphic to the 'fake' lens space which is the quotient $S^{3} / \mathbb{Z}_{p}$ by the free $\mathbb{Z}_{p}$-action

$$
\left(z_{1}, z_{2}\right) \longmapsto\left(\mathrm{e}^{2 \pi \mathrm{i} s / p} z_{1}, \mathrm{e}^{2 \pi \mathrm{i} / p} z_{2}\right)
$$

where $S^{3}$ is regarded as the unit sphere in $\mathbb{C}^{2}$. From the two-dimensional perspective, we will now show that the instanton expansion (5.36) retains topological information reflecting its five-dimensional origin, by rewriting it in terms of flat connection contributions to $\mathrm{U}(N)$ Chern-Simons gauge theory on the lens spaces $L(p, s)$.

Since

$$
\pi_{1}(L(p, s))=\mathbb{Z}_{p}
$$

gauge inequivalent flat $\mathrm{U}(N)$ connections are labelled by $N$-tuples $\vec{m} \in\left(\mathbb{Z}_{p}\right)^{N}$, which are torsion magnetic charges coming from the pullback of a Yang-Mills instanton on the sphere $S^{2}$ to a flat connection on $L(p, s)$ [17]. Let us then rewrite the series variables $\vec{\ell} \in \mathbb{Z}^{N}$ in $(5.36)$ as

$$
\vec{\ell}=\vec{m}+p \vec{l}
$$

with $\vec{l} \in \mathbb{Z}^{N}$. We can rewrite the interactions among eigenvalues from (5.35) as

$$
4 \sin \frac{\pi s}{p}\left(m_{j k}-t_{j k}+p\left(l_{j}-l_{k}\right)\right) \sin \pi\left(m_{j k}+t_{j k}+p\left(l_{j}-l_{k}\right)\right),
$$


and thus it depends only on the values of $\vec{\ell} \in \mathbb{Z}^{N}$ modulo $p$, that is, on $\vec{m} \in\left(\mathbb{Z}_{p}\right)^{N}$. ${ }^{14}$ They are also invariant under the Weyl symmetry group $S_{N}$ of the gauge group $\mathrm{U}(N)$, so that we can reduce the sum over all $N$-tuples $\vec{m} \in\left(\mathbb{Z}_{p}\right)^{N}$ to the ordered ones with

$$
m_{N} \geq m_{N-1} \geq \cdots \geq m_{1}
$$

Thus the partition function (5.36) depends only on how many times the integers $k \in$ $\{0,1, \ldots, p-1\}$ appear in the string $\left(m_{1}, \ldots, m_{N}\right)$. We denote these multiplicities as $\boldsymbol{N}=\left(N_{0}, N_{1}, \ldots, N_{p-1}\right)$, which by construction are $p$-component partitions of the $\operatorname{rank} N$ :

$$
N_{k} \geq 0 \quad \text { and } \quad \sum_{k=0}^{p-1} N_{k}=N .
$$

Under this reordering the Weyl symmetry breaks according to

$$
S_{N} \longrightarrow S_{N_{0}} \times S_{N_{1}} \times \cdots \times S_{N_{p-1}}
$$

The partition function (5.36) is then rewritten as

$$
\begin{aligned}
\mathcal{Z}_{N}(p, s)= & \left(\frac{s}{p}\right)^{N / 2} \sum_{N \vdash N} \frac{1}{\prod_{k=0}^{p-1} N_{k} !} \mathcal{W}_{p, s}\left(0^{N_{0}}, 1^{N_{1}}, \ldots,(p-1)^{N_{p-1}}\right) \\
& \times \sum_{\vec{l} \in \mathbb{Z}^{N}} \prod_{k=0}^{p-1} \exp \left(-\frac{2 \pi^{2}}{g_{\text {str }}} \sum_{j=N_{0}+N_{1} \cdots+N_{k-1}+1}^{N_{k}}\left(k+p l_{j}\right)^{2}\right),
\end{aligned}
$$

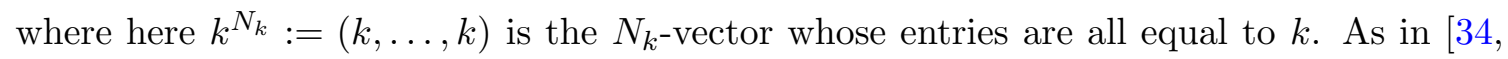
section 3.3], we identify in the second line a product of elliptic theta-functions

$$
\vartheta_{3}(\tau \mid z)=\sum_{l \in \mathbb{Z}} \mathrm{e}^{\pi \mathrm{i} \tau l^{2}+2 \pi \mathrm{i} l z}
$$

which enables us to write

$$
\begin{aligned}
\mathcal{Z}_{N}(p, s)= & \sum_{\boldsymbol{N} \vdash N} \mathrm{e}^{-\frac{2 \pi^{2}}{g_{\mathrm{str}}} \sum_{k=0}^{p-1} N_{k} k^{2}} \mathcal{W}_{p, s}\left(0^{N_{0}}, 1^{N_{1}}, \ldots,(p-1)^{N_{p-1}}\right) \\
& \times \prod_{k=0}^{p-1} \frac{\vartheta_{3}\left(\frac{2 \pi \mathrm{i} p^{2}}{g_{\mathrm{str}}} \mid \frac{2 \pi \mathrm{i} p k}{g_{\mathrm{str}}}\right)^{N_{k}}}{N_{k} !} .
\end{aligned}
$$

We can write the fluctuation weight $\mathcal{W}_{p, s}\left(0^{N_{0}}, 1^{N_{1}}, \ldots,(p-1)^{N_{p-1}}\right)$ explicitly in its integral form (5.35) and reorganize the integration variables $t_{j}$ into subsets $t_{j}^{J}$ with $J \in$ $\{0,1, \ldots, p-1\}$ and $j \in\left\{1, \ldots, N_{J}\right\}$. We then shift integration variables as $u_{j}^{J}:=t_{j}^{J}-j$

\footnotetext{
${ }^{14}$ Strictly speaking, this is only true if the integers $p$ and $s$ have the same even/odd parity, which we tacitly assume.
} 
to get

$$
\begin{aligned}
\mathcal{W}_{p, s}\left(0^{N_{0}}, 1^{N_{1}}, \ldots,(p-1)^{N_{p-1}}\right) \\
=\left(\frac{4 \pi}{g_{\mathrm{str}}}\right)^{N} \mathrm{e}^{\frac{g_{\mathrm{str}}}{12} N\left(N^{2}-1\right)\left(1+\frac{s^{2}}{p^{2}}\right)} \\
\quad \times \prod_{J=0}^{p-1} \int_{\mathbb{R}^{N_{J}}} \mathrm{~d} u^{J} \mathrm{e}^{-\frac{2 \pi^{2}}{g_{\mathrm{str}}} \sum_{j=1}^{N_{J}}\left(u_{j}^{J}+j\right)^{2}} \prod_{1 \leq j<k \leq N_{J}} 4 \sin \frac{\pi s}{p}\left(u_{j}^{J}-u_{k}^{J}\right) \sin \pi\left(u_{j}^{J}-u_{k}^{J}\right) \\
\quad \times \prod_{0 \leq J<K \leq p-1} \prod_{j=1}^{N_{J}} \prod_{k=1}^{N_{K}} 4 \sin \frac{\pi s}{p}\left(u_{j}^{J}-u_{k}^{K}+J-K\right) \sin \pi\left(u_{j}^{J}-u_{k}^{K}+J-K\right) .
\end{aligned}
$$

The products here which are independent of $(p, s)$ combine to give a standard Weyl determinant, while the $(p, s)$-dependent products carry the information about the surgery data of the Seifert homology sphere $X(s / p)$.

In fact, if we drop the product of theta-functions from the sum (5.48) and rescale the string coupling as before to $\tilde{g}_{\text {str }}=s g_{\text {str }} / p$, we can recognise the analytically continued partition function of $\mathrm{U}(N)$ Chern-Simons gauge theory at level $k \in \mathbb{Z}$ on the lens space $L(p, s)$ : the exponential prefactor is recognized as the classical contribution to the path integral from the Chern-Simons action evaluated on the flat $\mathrm{U}(N)$ connection labelled by $\boldsymbol{N}[17,81]$, with the analytic continuation

$$
\tilde{g}_{\mathrm{str}}=\frac{s g_{\mathrm{str}}}{p}=\frac{2 \pi \mathrm{i}}{k+N} .
$$

Moreover, after a straightforward change of integration variables (and subsequent analytic continuation), the integral expression (5.49) is easily seen to agree with the multi-eigenvalue integral formula from [82, Theorem 7] for the contribution to the one-loop fluctuation determinant from the flat connection $\boldsymbol{N}$. Thus the full partition function (5.48) can be written as

$$
\mathcal{Z}_{N}(p, s)=\sum_{N \vdash N} Z_{p, s}^{\mathrm{CS}}(\boldsymbol{N}) \prod_{k=0}^{p-1} \frac{\vartheta_{3}\left(\frac{2 \pi \mathrm{i} p^{2}}{g_{\mathrm{str}}} \mid \frac{2 \pi \mathrm{i} p k}{g_{\mathrm{str}}}\right)^{N_{k}}}{N_{k} !}
$$

where

$$
Z_{p, s}^{\mathrm{CS}}(\boldsymbol{N}):=\exp \left(\frac{2 \pi^{2} s}{\tilde{g}_{\mathrm{str}} p} \sum_{k=0}^{p-1} N_{k} k^{2}\right) \mathcal{W}_{p, s}\left(0^{N_{0}}, 1^{N_{1}}, \ldots,(p-1)^{N_{p-1}}\right)
$$

is the contribution to the Chern-Simons partition function from the point of the moduli space of flat connections on the lens space $L(p, s)$ labelled by

$$
\vec{m}=\left(0^{N_{0}}, 1^{N_{1}}, \ldots,(p-1)^{N_{p-1}}\right) .
$$

The connection between Chern-Simons theory on lens spaces $L(p, s)$ with $s>1$ and $q$-deformed Yang-Mills theory was also obtained in [17], but in a much different and more complicated fashion. There the two-dimensional gauge theory is defined on the collection of exceptional divisors of the four-dimensional Hirzebruch-Jung space $X_{4}(p, s)$, which is 
the minimal resolution of the $A_{p, s}$ singularity defined by the same orbifold action (5.39) on $\mathbb{C}^{2}$. The corresponding partition function depends explicitly on the intersection moduli $e_{i}$ of the exceptional divisors, which in the three-dimensional case translate into framing integers that enter the surgery construction of the Seifert space $L(p, s)$; after stripping away the Chern-Simons fluctuation determinants, the resulting partition function computes the contribution of fractional instantons to the partition function of topologically twisted $\mathcal{N}=4$ Yang-Mills theory on $X_{4}(p, s)$ [17]. This is not the case here. Like the topological ChernSimons theory, our two-dimensional gauge theory partition function (5.48) is independent of the framing integers $e_{i}$ and depends only on the pair of integers $(p, s)$ which uniquely determine $L(p, s)$ up to homeomorphism. In particular, stripping away the Chern-Simons fluctuation determinants $\mathcal{W}_{p, s}\left(0^{N_{0}}, 1^{N_{1}}, \ldots,(p-1)^{N_{p-1}}\right)$ would leave a $(p, s)$-independent partition function proportional to $\vartheta_{3}\left(\frac{2 \pi \mathrm{i}}{g_{\mathrm{str}}} \mid 0\right)^{N}$ [17], which is the contribution of fractional instantons to the partition function of $\mathcal{N}=4$ gauge theory on $X_{4}(1,1) \cong \mathcal{O}_{\mathbb{P}^{1}}(-1)$. This suggests that our squashing of the two-dimensional $q$-deformed gauge theory on $S^{2}$ is, like the standard theory at $p=1$, also related to the Calabi-Yau geometry of the resolved conifold $\mathcal{O}_{\mathbb{P}^{1}}(-1) \oplus \mathcal{O}_{\mathbb{P}^{1}}(-1)$; the topological string interpretation of this theory is certainly worthy of further investigation.

Large $\boldsymbol{N}$ limit. For any finite value of the $\operatorname{rank} N$, the partition function $\mathcal{Z}_{N}(b)$ is a continuous function of the squashing parameter $b>0$. In this sense our squashed $q$-deformations of two-dimensional Yang-Mills theory are continuations of the lens space theories analysed above: since the set of rational $b^{2}$ is dense in the space of all squashing parameters $b>0$, any partition function can be expressed as a limit of the two-dimensional gauge theories whose geometric meanings were explained above. It would be interesting to understand more precisely what the underlying geometry means for generic real values $b>0$.

However, we do expect the partition function (5.27) to experience a phase transition in the large $N$ regime, triggered by the discreteness of the matrix model, at least for large enough values of the squashing parameter $b$. The standard $q$-deformed Yang-Mills theory on $S^{2}$ undergoes a phase transition for $p>2$ [34], and we can extrapolate this to our more general setting. The eigenvalue distribution $\rho(\lambda)$ of a discrete random matrix ensemble is subject to the constraint

$$
\rho(\lambda) \leq 1
$$

which in the present case is always fulfilled at large $N$ when $b \leq \sqrt{2}$. It would be interesting to see how the phase transition appears at $b>\sqrt{2}$ in terms of the bi-orthogonal StieltjesWigert polynomials of [80, section 4.1]. It was argued in [80], and later proved in [83], that around the trivial flat connection the discrete and continuous versions of the StieltjesWigert ensemble are essentially the same, thus the zero-instanton sector of our squashing of $q$-deformed Yang-Mills theory can be obtained exactly via bi-orthogonal polynomials. For the case $b=1$ this gives the full partition function of $q$-deformed Yang-Mills theory, since the only flat connection on $L(1,1) \cong S^{3}$ is trivial. 
Introduce the 't Hooft coupling

$$
t:=g_{\mathrm{str}} N
$$

and take the double scaling limit $N \rightarrow \infty, g_{\mathrm{str}} \rightarrow 0$ with $t$ fixed. In this limit, the partition function (5.27) is proportional to the Chern-Simons matrix model on $L(p, 1)$ around the trivial connection, continued to $p=b^{2} \in \mathbb{R}$. Equivalently, from the instanton expansion (5.36) we infer that, as long as the fluctuations $\mathcal{W}_{b}(\vec{\ell})$ give sub-leading contributions, all instanton contributions are suppressed except for the trivial one. Taking the large $N$ limit of [80, eq. (2.26)], in the large $N$ regime we obtain

$$
\mathcal{Z}_{N}^{(\infty)}(b)=2^{N(N+1)}\left(\frac{2 \pi}{g_{\mathrm{str}}}\right)^{\frac{N}{2}} \exp \left(-\frac{N^{2} t}{12 b^{4}}\left(3 b^{8}+6 b^{4}-13\right)-\frac{N^{2} b^{8}}{t^{2}} F_{\mathrm{CS}}^{(0)}\left(\frac{t}{b^{4}}\right)\right)
$$

where

$$
F_{\mathrm{CS}}^{(0)}(t)=\frac{t^{3}}{12}-\frac{\pi^{2} t}{6}-\mathrm{Li}_{3}\left(\mathrm{e}^{-t}\right)+\zeta(3)
$$

is the planar free energy of Chern-Simons theory on $S^{3}$ with (analytically continued) 't Hooft coupling $t$. For $b \leq \sqrt{2}$ this solution is exact, and indeed the free energy of the supersymmetric gauge theory on $S_{b}^{3} \times S^{2}$ exhibits the $N^{3}$ behaviour in the strong coupling region for $t \rightarrow \infty$, as in the case of the five-sphere $S^{5}$ [77]. However, for higher values of $b$, this solution ceases to be valid for large $t$ and we expect the strong coupling region to have a different solution.

\subsection{Localization from seven dimensions}

With the premise of obtaining more general two-dimensional theories, we conclude by briefly commenting on how the derivation of $q$-deformed Yang-Mills theories would change if instead one started from a seven-dimensional cohomological gauge theory; as we argue, the story works in essentially the same way as in the five-dimensional case, though the physical significance of such a two-dimensional theory is not clear in this instance. For this, we consider our cohomological localization procedure for a seven-dimensional manifold $M_{7}=M_{5} \times \Sigma_{h}$. In order to have the right amount of supercharges in seven dimensions, we ought to start with a five-dimensional Seifert manifold admitting $\mathcal{N}=2$ supersymmetry, and henceforth we take the five-sphere $M_{5}=S^{5}$ for definiteness.

The seven-manifold $M_{5} \times \Sigma_{h}$ cannot come from a supergravity background, and we must pull back the Killing spinors on $M_{5}$ to seven dimensions. We define the theory on $S^{5} \times \mathbb{R}^{2}$ and build the components of a seven-dimensional Killing spinor from the tensor product of a five-dimensional Killing spinor with a constant spinor along $\mathbb{R}^{2}$. Schematically,

$$
\varepsilon^{(7)}=\varepsilon^{(5)} \otimes \zeta_{ \pm}^{(2)}
$$


Then we topologically twist the theory, and finally we put the cohomological field theory on $S^{5} \times \Sigma_{h} \cdot{ }^{15}$ The cohomological gauge theory in seven dimensions was constructed in [84] for the seven-sphere $S^{7}$, and extended in $[85,86]$ to other geometries. We do not review it here and refer to $[84,85]$ for the details. Our construction in seven dimensions differs from that of [84] in exactly the same manner as our construction in five dimensions differs from that of $[12,13]$. In particular, we pick a preferred vector field $v$ which generates the rotations along the $\mathrm{U}(1)$ fibre of $M_{5}$.

Again, the details of the supersymmetry transformations and localization locus do not depend on the specific details of the geometry given (although the solutions do depend on it) and we simply reproduce step by step the procedure of [84, section 5] and [85, section 4]. Fortunately, most of the steps in the construction of a cohomological field theory in [84] rely only on the Seifert structure and not on the K-contact structure, and can be adapted to our framework with minor changes. The localization locus has a complicated expression, and we only focus here on the perturbative part, hence expanding around the trivial connection. For the vector multiplet, we have to study the same ratio of fluctuation determinants as in [85, eqs. (4.18)-(4.19)], which has the form

$$
h(\phi)=\frac{\left.\left.\operatorname{det} i \mathrm{~L}_{\phi}\right|_{\Omega_{H}^{(0,0)}\left(M_{7}, \mathfrak{g}\right)} \operatorname{det} \mathrm{iL}_{\phi}\right|_{\Omega_{H}^{(0,2)}\left(M_{7}, \mathfrak{g}\right)}}{\left.\left.\operatorname{det} \mathrm{i} \mathrm{L}_{\phi}\right|_{\Omega_{H}^{(0,1)}\left(M_{7}, \mathfrak{g}\right)} \operatorname{det} \mathrm{i} \mathrm{L}_{\phi}\right|_{\Omega_{H}^{(0,3)}\left(M_{7}, \mathfrak{g}\right)}},
$$

where the differential operator $\mathrm{L}_{\phi}$ is, as usual, the sum of a Lie derivative $\mathcal{L}_{v}$ along $v$ and a gauge transformation $\mathcal{G}_{\phi}$. This ratio of determinants can be computed using the same strategy as in five dimensions. In fact, the product geometry $S^{5} \times \Sigma_{h}$ is simpler than, for example $S^{7}$, since we know how to decompose the vector space of differential forms into eigenmodes of the Lie derivative $\mathcal{L}_{v}$. For $M_{7}=S^{5} \times \Sigma_{h}$, this is exactly the decomposition of [12] on $S^{5}$, which is classified by powers of the line bundle $\mathscr{L}$ on the projective plane $\mathbb{P}^{2}$ associated to the Seifert fibration $S^{5} \rightarrow \mathbb{P}^{2}$. At the end of the day, the number of remaining modes is counted by the index of the twisted Dolbeault complex

$$
\begin{aligned}
\Omega^{(0,0)}\left(\mathbb{P}^{2} \times \Sigma_{h}\right)_{m, \alpha} \stackrel{\bar{\partial}^{(m)}}{\longrightarrow} \Omega^{(0,1)}\left(\mathbb{P}^{2} \times \Sigma_{h}\right)_{m, \alpha} \stackrel{\bar{\partial}^{(m)}}{\longrightarrow} \Omega^{(0,2)}\left(\mathbb{P}^{2} \times \Sigma_{h}\right)_{m, \alpha} \\
\stackrel{\bar{\partial}^{(m)}}{\longrightarrow} \Omega^{(0,3)}\left(\mathbb{P}^{2} \times \Sigma_{h}\right)_{m, \alpha},
\end{aligned}
$$

where we adopted the shorthand notation

$$
\Omega^{(\bullet, \bullet)}\left(\mathbb{P}^{2} \times \Sigma_{h}\right)_{m, \alpha}:=\Omega^{(\bullet, \bullet)}\left(\mathbb{P}^{2} \times \Sigma_{h}, \mathscr{L}^{\otimes m} \otimes \mathfrak{g}_{\alpha}\right) .
$$

We then arrive at

$$
Z_{\text {vec }}^{\text {pert }}\left(S^{5} \times \Sigma_{h}\right)=\prod_{\alpha \in \triangle} \prod_{m \neq 0}\left(\frac{m}{r}-\mathrm{i}(\alpha, \phi)\right)^{\operatorname{index} \bar{\partial}^{(m)}} .
$$

\footnotetext{
${ }^{15} \mathrm{Had}$ we partially twisted the theory along $\Sigma_{h}$, we would have obtained a localization locus which is the same as for the purely five-dimensional theory. The main difference between the seven-dimensional and the five-dimensional setting with partial twist would be the following. For the case $S^{3} \times \Sigma_{h}$ the localization locus consisted only of the trivial connection on $S^{3}$, thus effectively reducing to a two-dimensional theory. For $S^{5} \times \Sigma_{h}$, on the contrary, we would obtain a sum of copies of two-dimensional theories, each one in a different background. Such backgrounds for the two-dimensional theory are contact instantons on $S^{5}$, which descend to anti-self-dual instantons on $\mathbb{P}^{2}$. We do not pursue the partial twist description here.
} 
The index is computed as follows. The total Chern class of the holomorphic tangent bundle of the base $K_{6}=\mathbb{P}^{2} \times \Sigma_{h}$ of the seven-dimensional Seifert fibration is

$$
\begin{aligned}
c\left(T^{1,0} K_{6}\right)= & c\left(T^{1,0} \mathbb{P}^{2}\right) \wedge c\left(T^{1,0} \Sigma_{h}\right) \\
= & \left(1+\omega_{\mathbb{P}^{2}}\right)^{\wedge 3} \wedge\left(1+\chi\left(\Sigma_{h}\right) \omega_{\Sigma_{h}}\right) \\
= & 1+\left(3 \omega_{\mathbb{P}^{2}}+\chi\left(\Sigma_{h}\right) \omega_{\Sigma_{h}}\right)+3\left(\omega_{\mathbb{P}^{2}} \wedge \omega_{\mathbb{P}^{2}}+\chi\left(\Sigma_{h}\right) \omega_{\mathbb{P}^{2}} \wedge \omega_{\Sigma_{h}}\right) \\
& +3 \omega_{\mathbb{P}^{2}} \wedge \omega_{\mathbb{P}^{2}} \wedge \omega_{\Sigma_{h}} \\
= & 1+c_{1}\left(T^{1,0} K_{6}\right)+c_{2}\left(T^{1,0} K_{6}\right)+c_{3}\left(T^{1,0} K_{6}\right),
\end{aligned}
$$

which gives the corresponding Todd class

$$
\begin{aligned}
\operatorname{Td}\left(T^{1,0} K_{6}\right)= & 1+\frac{1}{2} c_{1}\left(T^{1,0} K_{6}\right)+\frac{1}{12}\left(c_{1}\left(T^{1,0} K_{6}\right) \wedge c_{1}\left(T^{1,0} K_{6}\right)+c_{2}\left(T^{1,0} K_{6}\right)\right) \\
& +\frac{1}{24} c_{1}\left(T^{1,0} K_{6}\right) \wedge c_{2}\left(T^{1,0} K_{6}\right) \\
= & 1+\frac{3}{2} \omega_{\mathbb{P}^{2}}+(1-h) \omega_{\Sigma_{h}}+\omega_{\mathbb{P}^{2}} \wedge \omega_{\mathbb{P}^{2}}+\frac{3}{2}(1-h) \omega_{\mathbb{P}^{2}} \wedge \omega_{\Sigma_{h}} \\
& +(1-h) \omega_{\mathbb{P}^{2}} \wedge \omega_{\mathbb{P}^{2}} \wedge \omega_{\Sigma_{h}} .
\end{aligned}
$$

Since $c_{1}(\mathscr{L})=\omega_{\mathbb{P}^{2}}$, the Chern character of the line bundle $\mathscr{L}^{\otimes m}$ is

$$
\begin{aligned}
\operatorname{ch}\left(\mathscr{L}^{\otimes m}\right)= & 1+c_{1}\left(\mathscr{L}^{\otimes m}\right)+\frac{1}{2} c_{1}\left(\mathscr{L}^{\otimes m}\right) \wedge c_{1}\left(\mathscr{L}^{\otimes m}\right) \\
& +\frac{1}{6} c_{1}\left(\mathscr{L}^{\otimes m}\right) \wedge c_{1}\left(\mathscr{L}^{\otimes m}\right) \wedge c_{1}\left(\mathscr{L}^{\otimes m}\right) \\
= & 1+m \omega_{\mathbb{P}^{2}}+\frac{1}{2} m^{2} \omega_{\mathbb{P}^{2}} \wedge \omega_{\mathbb{P}^{2}} .
\end{aligned}
$$

A simple computation as in the five-dimensional setting then gives

$$
\left.\operatorname{index} \bar{\partial}^{(m)}\right|_{\mathbb{P}^{2} \times \Sigma_{h}}=(1-h)\left(1+\frac{3}{2} m+\frac{1}{2} m^{2}\right)=\left.(1-h) \operatorname{index} \bar{\partial}^{(m)}\right|_{\mathbb{P}^{2}} .
$$

We conclude that the computation of the one-loop determinant of the vector multiplet on $S^{5}$ is lifted to $S^{5} \times \Sigma_{h}$ in exactly the same way as the computation on $S^{3}$ is lifted to $S^{3} \times \Sigma_{h}$. The resulting contribution to the partition function yields polylogarithmic corrections to the standard $q$-deformed measure [12], and in this case (5.62) evaluates to

$$
Z_{\text {vec }}^{\text {pert }}\left(S^{5} \times \Sigma_{h}\right)=\prod_{\alpha \in \triangle_{+}}\left(\frac{\sinh (\pi r(\alpha, \phi))^{2}}{(\pi r(\alpha, \phi))^{2}} \mathrm{e}^{f(\pi r(\alpha, \phi)) / \pi^{2}}\right)^{1-h},
$$

where the function $f$ is defined by

$$
f(x)=-\frac{x^{3}}{3}-x^{2} \log \left(1-\mathrm{e}^{2 x}\right)-x \operatorname{Li}_{2}\left(\mathrm{e}^{2 x}\right)+\frac{1}{2} \operatorname{Li}_{3}\left(\mathrm{e}^{2 x}\right)-\frac{\zeta(3)}{2} .
$$




\section{Acknowledgments}

We thank Luca Griguolo, Sara Pasquetti and Domenico Seminara for helpful discussions. The work of L.S. was supported by the Doctoral Scholarship SFRH/BD/129405/2017 from the Fundação para a Ciência e a Tecnologia (FCT). The work of L.S. and M.T. was supported by the FCT Project PTDC/MAT-PUR/30234/2017. The work of R.J.S. was supported by the Consolidated Grant ST/P000363/1 from the U.K. Science and Technology Facilities Council (STFC).

\section{A Spinor conventions}

For field theories in three dimensions we follow the normalization and conventions of [11]. We work in the $\mathcal{N}=2$ formalism, with $\mathrm{SU}(2)_{R}$ R-symmetry, and for theories with $\mathcal{N}=4$ supersymmetry only $\mathrm{SU}(2)_{R} \subset \mathrm{SU}(2)_{C} \times \mathrm{SU}(2)_{H}$ is manifest. In five dimensions, we follow [13]. We work in the $\mathcal{N}=1$ formalism, with $\mathrm{SU}(2)_{R}$ R-symmetry, using the letters $I, J, \ldots$ for the indices. In theories admitting $\mathcal{N}=2$ supersymmetry, only $\mathrm{SU}(2)_{R} \subset$ $\mathrm{SU}(2)_{R} \times \mathrm{U}(1)_{r} \subset \mathrm{SO}(5)_{R}$ is manifest, where $\mathrm{SU}(2)_{R} \times \mathrm{U}(1)_{r}$ is the maximal R-symmetry group preserved by the product manifolds considered in the main text and $\mathrm{SO}(5)_{R}$ the R-symmetry in five-dimensional flat space. In both three and five dimensions, $\mathrm{SU}(2)_{R}$ indices are raised and lowered with the Levi-Civita symbol $\epsilon_{I J}$ or $\epsilon^{I J}$, with the convention $\epsilon_{12}=-1=-\epsilon^{12}$.

We do not write Lorentz spin indices explicitly in the spinors, and understand that they are contracted using the charge conjugation matrix $C$, a real antisymmetric matrix satisfying

$$
C \Gamma^{\mu}=\left(\Gamma^{\mu}\right)^{\top} C
$$

With this choice, spinor components are taken to be Grassmann-even, and anticommutation is a consequence of $C^{\top}=-C$. This is also in agreement with the conventions in [48, 49], where Killing spinors from rigid supergravity are taken to be Grassmann-even symplectic Majorana spinors. Our notation for Killing spinors is then as follows: $\varepsilon^{I}$ satisfies

$$
\left(\epsilon_{I J} C \varepsilon^{J}\right)^{*}=\varepsilon^{I}
$$

with $C$ the charge conjugation matrix. This implies

$$
\varepsilon_{I}^{\dagger}=\left(\epsilon_{I J} \varepsilon^{J}\right)^{\top}
$$

We impose the following reality conditions on the fields in the five-dimensional theories. The scalars $\left(\mathrm{q}^{\dagger}\right)^{I}$ in a hypermultiplet are related to $\mathrm{q}_{I}$ by complex conjugation and transposition. As we are working in Euclidean space, there is no reality condition on the spinor fields, and we have to choose a half-dimensional integration cycle in the configuration space of fields. The gauginos $\lambda^{I}$ are symplectic Majorana spinors,

$$
\left(\epsilon_{I J} C \lambda^{J}\right)^{*}=\lambda^{I}
$$


and we take as a definition of the fields $\lambda_{I}^{\dagger}$ the equation

$$
\lambda_{I}^{\dagger}=\left(\epsilon_{I J} \lambda^{J}\right)^{\top}
$$

The reasoning for the spinor $\psi$ in a hypermultiplet is analogous. See also [53, section 2.1] for discussion about the treatment of the reality condition for spinors. The sole difference between our conventions and those of $[13,53]$ is a factor $\sqrt{-1}$ in the definition of the scalar $\sigma$ in the vector multiplet, as in those references the rotated field (which we denote $\sigma_{0}$ ) is taken from the very beginning.

In three dimensions, the gauginos $\lambda^{I}$ are not subject to additional constraints, and we impose $\lambda_{I}^{\dagger}$ to be related to $\lambda^{I}$ as in Minkowski signature, following [11, 54], and similarly for $\psi^{\dagger}$ and $\psi$.

\section{B Squashed three-spheres}

Three types of squashed sphere $S_{b}^{3}$ that preserve at least $\mathcal{N}=2$ supersymmetry (four supercharges) exist in the literature: the squashed sphere called the "familiar case" in [28], the squashed sphere of [29], and the ellipsoid of [28] which was originally called the "less familiar case" of squashed sphere. We have ordered them according to their increasing deviation from the standard round sphere $S^{3}$. In the following we briefly describe and discuss them within the cohomological field theory formalism, see also [15, section 7] for related discussion.

The simplest case of squashed sphere is the "familiar case" of [28], for which the oneloop determinants are the same as in the round case up to rescaling of variables. This squashed sphere is obtained by simply changing the radius of the Hopf fibre with respect to the radius of the base $S^{2}$, so the background has isometry group $\mathrm{SU}(2) \times \mathrm{U}(1)$. The Killing spinor is covariantly constant, as on the round $S^{3}$. The Killing vector field $v$ has compact orbits and coincides with the generator of rotations along the Hopf fibre, hence it is parallel to the Reeb vector field $\xi$. The computation of the one-loop determinants on this geometry is very simple in our setting: it is clear from construction (see (3.25)-(3.28)) that only the radius of the circle fibre enters the one-loop determinant, and the result of [28] follows immediately from Källén's formula. More generally, the one-loop determinants on the squashed lens spaces $S_{b}^{3} / \mathbb{Z}_{p}$, with $p \in \mathbb{Z}_{>0}$ and $S_{b}^{3}$ the "familiar" squashed sphere of [28], are given by the same formula as for the round lens space, with the proper scaling of the size of the fibre.

Another squashing that preserves $\mathcal{N}=2$ supersymmetry is the non-trivial squashed sphere of [29]. This is obtained by twisted dimensional reduction from $S^{3} \times \mathbb{R}$ with round $S^{3}$. One employs a Scherk-Schwarz compactification to put the theory on $S^{3} \times S^{1}$ by identifying

$$
\exp \left(2 \pi \beta \frac{\partial}{\partial t}+\pi \beta \mathcal{R}\right) X \sim X
$$

for any field $X$, where $\beta$ is the radius of $S^{1}, t$ is the coordinate along $\mathbb{R}$ and $\mathcal{R}$ is the generator of an R-symmetry transformation. This R-symmetry, which differs from the 
R-symmetry at the superconformal fixed point, twists the compactification by including a finite rotation on $S^{3}$ in the periodic identification of fields along $S^{1}$, and hence also twists the dimensional reduction when sending $\beta \rightarrow 0$. With this procedure, one is able to preserve an $\mathrm{SU}(2) \times \mathrm{U}(1)$ isometry group and to obtain a metric on $S^{3}$ as for the "familiar case" of [28], hence it does not modify the metric on the base $S^{2}$ of the Hopf fibration. However, the Killing spinor now becomes a non-constant field. In [46, section 5.2] it was shown that the supergravity background of [29] admits $\mathcal{N}=4$ supersymmetry. The Killing vector field $v$ has closed orbits, and therefore Källén's localization directly applies. In this squashed sphere, the Killing spinor depends on a parameter $u$ related to the radius of the circle fibre through

$$
\frac{r^{2}}{\epsilon^{2}}=1+u^{2},
$$

where $r$ is the radius of the round base $S^{2}$ and $\epsilon$ is the radius of the $\mathrm{U}(1)$ fibre. This relation arises from the twisted dimensional reduction. Since the Killing vector field is non-constant, although it points along the squashed Hopf fibre one has to consider the complex dependence on $1 \pm \mathrm{i} u$ : at the two fixed points the fibre has radius, respectively, given by

$$
\epsilon_{1}=r b \quad \text { and } \quad \epsilon_{2}=r b^{-1} \quad \text { with } \quad b=\sqrt{\frac{1-\mathrm{i} u}{1+\mathrm{i} u}} .
$$

This justifies the applicability of the localization formula (3.28), and explains why the one-loop determinants with this non-trivial squashing are formally the same as for the ellipsoid of [28].

Finally we consider the ellipsoid of [28], which is the squashed sphere considered mostly in the main text. It has only $\mathrm{U}(1) \times \mathrm{U}(1)$ isometry group as the squashing also deforms the metric on the base $S^{2}$ of the fibration. It is defined as the locus in $\mathbb{C}^{2}$ satisfying

$$
b^{2}\left|z_{1}\right|^{2}+b^{-2}\left|z_{2}\right|^{2}=r^{2}
$$

with $b=\sqrt{\epsilon_{1} / \epsilon_{2}}$ and $r=\sqrt{\epsilon_{1} \epsilon_{2}}$. The supergravity background only preserves four supercharges. As explained in [46, section 5.1] and highlighted in the main text, the orbits of the Killing vector field $v$ are not closed, and $v$ does not point along the fibre direction. It splits into two vector fields, generating two $\mathrm{U}(1)$ isometries with closed orbits. The localized partition function on this geometry is discussed in section 3.5.

Notice the different geometric meaning of the squashing parameter $b$ in the squashed sphere [29] and in the ellipsoid [28]. For the ellipsoid $b>0$ is real, while for the squashed sphere $b \in \mathbb{C}$ with $|b|=1$. In both cases the partition functions can be analytically continued to arbitrary complex values $b \in \mathbb{C} \backslash\{0\}$, and the expressions are given by (3.43) and (3.46). Thus, in practice, we can compute the one-loop determinants on the regular background $S_{b}^{3}$ of [29] and then continue the result to $b>0$, or vice versa. The limit $b \rightarrow 0^{+}$in the squashed sphere and ellipsoid also has different geometric meaning. For the squashed sphere, $b \rightarrow 0^{+}$means $u \rightarrow-\mathrm{i}$, and corresponds to blowing up the twisted Hopf fibre. In the ellipsoid, $b \rightarrow 0^{+}$reduces the geometric locus (B.4) to $\left|z_{2}\right|=0$, hence the $S_{b}^{3}$ geometry degenerates to $\mathbb{C}$. In both cases the geometry becomes non-compact, and we do not expect the cohomological localization to work in this limit. 


\section{Sasaki-Einstein five-manifolds}

There exists a family of Sasaki-Einstein metrics on five-manifolds $M_{5}$ which topologically are U(1)-fibrations over the product $S^{2} \times S^{2}$ [87]. The simplest case is the familiar conifold $T^{1,1}$, which is homeomorphic to $S^{3} \times S^{2}$ and so its associated line bundle $\mathscr{L}_{1,1}$ has Chern class $c_{1}\left(\mathscr{L}_{1,1}\right)=\omega_{1}$ given by the generator $\left[\omega_{1}\right] \in H^{2}\left(S^{2}, \mathbb{Z}\right)$ of the first base factor. More generally, there is an infinite family of irregular backgrounds labeled by a pair of integers $(p, s)$, and denoted $Y^{p, s}[88]$. The first Chern class associated to the circle bundle $Y^{p, s} \rightarrow$ $S^{2} \times S^{2}$ is

$$
c_{1}\left(\mathscr{L}_{p, s}\right)=p \omega_{1}+s \omega_{2}
$$

where $\left[\omega_{1}\right]$ and $\left[\omega_{2}\right]$ generate the second cohomology $H^{2}\left(S^{2}, \mathbb{Z}\right)$ of the respective factors of the base. When $p$ and $s$ are coprime, $Y^{p, s}$ is again topologically $S^{3} \times S^{2}$.

Field theories with $\mathcal{N}=1$ supersymmetry on these manifolds have been studied in [18, 19] via application of the index theorem. Except for the simplest case $S^{3} \times S^{2}$, the Killing vector field $v$ does not have closed orbits. Moreover, the Reeb vector field $\xi$ does not act freely on the base space of the fibration, which is a warped product $S^{2} \rtimes S^{2}$. These manifolds have a toric action, and in fact admit a free $\mathrm{U}(1)$ action, which however is generated by a different vector field from the Reeb vector field. Therefore the formalism of this paper does not apply. In [18], the way around this problem was to use the same idea that we did, but in the opposite direction. The manifolds $Y^{p, s}$ can be obtained as a quotient $S^{3} \times S^{3} / \mathrm{U}(1)$, with U(1) acting freely on the six-dimensional manifold $S^{3} \times S^{3}$. One can compute the one-loop determinants using the index of the twisted Dolbeault complex in six dimensions, and then use the fact that there is a free $\mathrm{U}(1)$ action to push it down to $Y^{p, s}$. In the case of the conifold $T^{1,1}$, the vacuum moduli spaces of instantons have been described in this way by [89].

In fact, the most natural way to look at these geometries is as follows. Consider $\mathbb{C}^{4}$ as the direct product $\mathbb{C}^{2} \times \mathbb{C}^{2}$, with a sphere $S^{3} \subset \mathbb{C}^{2}$ embedded in each factor in the standard way. There is a $\mathrm{U}(1)^{\times 4}$ action on $\mathbb{C}^{4}$, where each $\mathrm{U}(1)_{i}$ acts on the corresponding factor of $\mathbb{C}$ in $\mathbb{C}^{4}$, with associated equivariant parameter $\epsilon_{i}^{-1}, i=1, \ldots, 4$. There is a further $\mathrm{U}(1)_{T}$ action on $\mathbb{C}^{4}$ with charges which are functions of two integer parameters $p$ and $s$; explicitly, $\mathrm{U}(1)_{T}$ acts with charges $(p+s, p-s,-p,-s)$. Then the action of $\mathrm{U}(1)_{T}$ is free on $S^{3} \times S^{3} \subset \mathbb{C}^{4}$, and taking the quotient $S^{3} \times S^{3} / \mathrm{U}(1)_{T}$ gives $Y^{p, s}$ with a residual toric action by $\mathrm{U}(1)^{\times 3}$, but none of the remaining $\mathrm{U}(1)$ actions is free. The one-loop determinants then appear as products over four integers $m_{i}, i=1, \ldots, 4$, each one corresponding to an eigenvalue $\frac{m_{i}}{\epsilon_{i}}$ where $\epsilon_{i}^{-1}$ is the equivariant parameter for rotation in the $i$-th plane $\mathbb{C}$ in $\mathbb{C}^{4}$. These four integers are constrained by one linear relation, corresponding to the quotient by the freely acting $\mathrm{U}(1)_{T}$, which reads

$$
(p+s) m_{1}+(p-s) m_{2}-p m_{3}-s m_{4}=0 .
$$

Open Access. This article is distributed under the terms of the Creative Commons Attribution License (CC-BY 4.0), which permits any use, distribution and reproduction in any medium, provided the original author(s) and source are credited. 


\section{References}

[1] M.R. Douglas, S.H. Katz and C. Vafa, Small instantons, Del Pezzo surfaces and type-I-prime theory, Nucl. Phys. B 497 (1997) 155 [hep-th/9609071] [INSPIRE].

[2] K.A. Intriligator, D.R. Morrison and N. Seiberg, Five-dimensional supersymmetric gauge theories and degenerations of Calabi-Yau spaces, Nucl. Phys. B 497 (1997) 56 [hep-th/9702198] [INSPIRE].

[3] L. Bhardwaj, Dualities of 5d gauge theories from S-duality, arXiv:1909.05250 [INSPIRE].

[4] R. Gopakumar and C. Vafa, M theory and topological strings. 2, hep-th/9812127 [INSPIRE].

[5] D. Gaiotto, $N=2$ dualities, JHEP 08 (2012) 034 [arXiv:0904.2715] [INSPIRE].

[6] D. Gaiotto, G.W. Moore and A. Neitzke, Wall-crossing, Hitchin systems and the WKB approximation, arXiv:0907.3987 [INSPIRE].

[7] A. Gadde, L. Rastelli, S.S. Razamat and W. Yan, The 4d superconformal index from q-deformed 2d Yang-Mills, Phys. Rev. Lett. 106 (2011) 241602 [arXiv:1104.3850] [InSPIRE].

[8] M.R. Douglas, On D = 5 super Yang-Mills theory and (2,0) theory, JHEP 02 (2011) 011 [arXiv: 1012.2880] [INSPIRE].

[9] N. Lambert, C. Papageorgakis and M. Schmidt-Sommerfeld, M5-branes, D4-branes and quantum 5D super-Yang-Mills, JHEP 01 (2011) 083 [arXiv:1012.2882] [INSPIRE].

[10] Y. Fukuda, T. Kawano and N. Matsumiya, 5D SYM and 2D q-deformed YM, Nucl. Phys. B 869 (2013) 493 [arXiv: 1210.2855] [INSPIRE].

[11] J. Kallen, Cohomological localization of Chern-Simons theory, JHEP 08 (2011) 008 [arXiv: 1104.5353] [INSPIRE].

[12] J. Källén and M. Zabzine, Twisted supersymmetric 5D Yang-Mills theory and contact geometry, JHEP 05 (2012) 125 [arXiv:1202.1956] [INSPIRE].

[13] J. Källén, J. Qiu and M. Zabzine, The perturbative partition function of supersymmetric $5 D$ Yang-Mills theory with matter on the five-sphere, JHEP 08 (2012) 157 [arXiv:1206.6008] [INSPIRE].

[14] N. Drukker, T. Okuda and F. Passerini, Exact results for vortex loop operators in $3 d$ supersymmetric theories, JHEP 07 (2014) 137 [arXiv:1211.3409] [INSPIRE].

[15] T. Kawano and N. Matsumiya, 5D SYM on 3D deformed spheres, Nucl. Phys. B 898 (2015) 456 [arXiv: 1505.06565] [INSPIRE].

[16] P.M. Crichigno, D. Jain and B. Willett, 5d partition functions with a twist, JHEP 11 (2018) 058 [arXiv: 1808.06744] [INSPIRE].

[17] L. Griguolo, D. Seminara, R.J. Szabo and A. Tanzini, Black holes, instanton counting on toric singularities and q-deformed two-dimensional Yang-Mills theory, Nucl. Phys. B 772 (2007) 1 [hep-th/0610155] [INSPIRE].

[18] J. Qiu and M. Zabzine, 5D super Yang-Mills on $Y^{p, q}$ Sasaki-Einstein manifolds, Commun. Math. Phys. 333 (2015) 861 [arXiv:1307.3149] [INSPIRE].

[19] J. Qiu and M. Zabzine, Factorization of $5 D$ super Yang-Mills theory on $Y^{p, q}$ spaces, Phys. Rev. D 89 (2014) 065040 [arXiv: 1312.3475] [INSPIRE].

[20] D. Gaiotto, L. Rastelli and S.S. Razamat, Bootstrapping the superconformal index with surface defects, JHEP 01 (2013) 022 [arXiv: 1207.3577] [INSPIRE]. 
[21] O. Aharony et al., The Hagedorn-deconfinement phase transition in weakly coupled large $N$ gauge theories, Adv. Theor. Math. Phys. 8 (2004) 603 [hep-th/0310285] [InSPIRE].

[22] J. Kinney, J.M. Maldacena, S. Minwalla and S. Raju, An index for 4 dimensional super conformal theories, Commun. Math. Phys. 275 (2007) 209 [hep-th/0510251] [INSPIRE].

[23] F.A.H. Dolan, V.P. Spiridonov and G.S. Vartanov, From 4d superconformal indices to $3 d$ partition functions, Phys. Lett. B 704 (2011) 234 [arXiv:1104.1787] [INSPIRE].

[24] A. Gadde and W. Yan, Reducing the 4d index to the $S^{3}$ partition function, JHEP 12 (2012) 003 [arXiv: 1104.2592] [INSPIRE].

[25] Y. Imamura, Relation between the $4 d$ superconformal index and the $S^{3}$ partition function, JHEP 09 (2011) 133 [arXiv:1104.4482] [INSPIRE].

[26] N.A. Nekrasov, Instanton partition functions and M-theory, Jpn. J. Math. 4 (2009) 63.

[27] N. Seiberg, Five-dimensional SUSY field theories, nontrivial fixed points and string dynamics, Phys. Lett. B 388 (1996) 753 [hep-th/9608111] [INSPIRE].

[28] N. Hama, K. Hosomichi and S. Lee, SUSY gauge theories on squashed three-spheres, JHEP 05 (2011) 014 [arXiv: 1102.4716] [InSPIRE].

[29] Y. Imamura and D. Yokoyama, $N=2$ supersymmetric theories on squashed three-sphere, Phys. Rev. D 85 (2012) 025015 [arXiv:1109.4734] [InSPIRE].

[30] A. Gadde, L. Rastelli, S.S. Razamat and W. Yan, Gauge theories and macdonald polynomials, Commun. Math. Phys. 319 (2013) 147 [arXiv:1110.3740] [InSPIRE].

[31] Y. Tachikawa, 4d partition function on $S^{1} \times S^{3}$ and $2 d$ Yang-Mills with nonzero area, PTEP 2013 (2013) 013B01 [arXiv: 1207.3497] [INSPIRE].

[32] M. Aganagic, H. Ooguri, N. Saulina and C. Vafa, Black holes, q-deformed 2d Yang-Mills and non-perturbative topological strings, Nucl. Phys. B 715 (2005) 304 [hep-th/0411280] [INSPIRE].

[33] M. Blau and G. Thompson, Chern-Simons theory on S1-bundles: abelianisation and q-deformed Yang-Mills theory, JHEP 05 (2006) 003 [hep-th/0601068] [INSPIRE].

[34] N. Caporaso et al., Topological strings and large $N$ phase transitions. I. Nonchiral expansion of q-deformed Yang-Mills theory, JHEP 01 (2006) 035 [hep-th/0509041] [InSPIRE].

[35] S.S. Razamat and B. Willett, Star-shaped quiver theories with flux, Phys. Rev. D 101 (2020) 065004 [arXiv: 1911.00956] [INSPIRE].

[36] J. Yagi, 3d TQFT from 6d SCFT, JHEP 08 (2013) 017 [arXiv: 1305.0291] [INSPIRE].

[37] S. Lee and M. Yamazaki, 3d Chern-Simons theory from M5-branes, JHEP 12 (2013) 035 [arXiv: 1305.2429] [INSPIRE].

[38] D. Gang, N. Kim, M. Romo and M. Yamazaki, Aspects of defects in $3 d-3 d$ correspondence, JHEP 10 (2016) 062 [arXiv: 1510.05011] [INSPIRE].

[39] C. Cordova and D.L. Jafferis, Complex Chern-Simons from M5-branes on the squashed three-sphere, JHEP 11 (2017) 119 [arXiv: 1305.2891] [INSPIRE].

[40] R.J. Szabo, Equivariant localization of path integrals, Lect. Notes Phys. Monogr. 63 (2000) 1 [hep-th/9608068] [INSPIRE].

[41] V. Pestun and M. Zabzine, Introduction to localization in quantum field theory, J. Phys. A 50 (2017) 443001 [arXiv: 1608.02953] [INSPIRE]. 
[42] K. Ohta and Y. Yoshida, Non-Abelian localization for supersymmetric Yang-Mills-Chern-Simons theories on Seifert manifold, Phys. Rev. D 86 (2012) 105018 [arXiv:1205.0046] [INSPIRE].

[43] V. Pestun, Localization of gauge theory on a four-sphere and supersymmetric Wilson loops, Commun. Math. Phys. 313 (2012) 71 [arXiv:0712.2824] [INSPIRE].

[44] D.L. Jafferis and S.S. Pufu, Exact results for five-dimensional superconformal field theories with gravity duals, JHEP 05 (2014) 032 [arXiv:1207.4359] [INSPIRE].

[45] G. Festuccia and N. Seiberg, Rigid Supersymmetric Theories in Curved Superspace, JHEP 06 (2011) 114 [arXiv:1105.0689] [INSPIRE].

[46] C. Closset, T.T. Dumitrescu, G. Festuccia and Z. Komargodski, Supersymmetric field theories on three-manifolds, JHEP 05 (2013) 017 [arXiv:1212.3388] [INSPIRE].

[47] L.F. Alday, D. Martelli, P. Richmond and J. Sparks, Localization on three-manifolds, JHEP 10 (2013) 095 [arXiv: 1307.6848] [INSPIRE].

[48] Y. Pan, Rigid supersymmetry on 5-dimensional Riemannian manifolds and contact geometry, JHEP 05 (2014) 041 [arXiv: 1308.1567] [INSPIRE].

[49] L.F. Alday et al., Supersymmetric gauge theories on five-manifolds, JHEP 08 (2015) 007 [arXiv: 1503.09090] [INSPIRE].

[50] C. Closset and H. Kim, Three-dimensional $\mathcal{N}=2$ supersymmetric gauge theories and partition functions on Seifert manifolds: a review, Int. J. Mod. Phys. A 34 (2019) 1930011 [arXiv: 1908.08875] [INSPIRE].

[51] J. Qiu and M. Zabzine, On twisted $N=25 D$ super Yang-Mills theory, Lett. Math. Phys. 106 (2016) 1 [arXiv: 1409.1058] [INSPIRE].

[52] M. Blau and G. Thompson, Lectures on $2-D$ gauge theories: Topological aspects and path integral techniques, ICTP Ser. Theor. Phys. 10 (1994) 175 [hep-th/9310144] [INSPIRE].

[53] J. Qiu and M. Zabzine, Review of localization for $5 d$ supersymmetric gauge theories, J. Phys. A 50 (2017) 443014 [arXiv: 1608.02966] [INSPIRE].

[54] A. Kapustin, B. Willett and I. Yaakov, Exact results for Wilson loops in superconformal Chern-Simons theories with matter, JHEP 03 (2010) 089 [arXiv:0909.4559] [INSPIRE].

[55] N. Caporaso et al., Topological strings, two-dimensional Yang-Mills theory and Chern-Simons theory on torus bundles, Adv. Theor. Math. Phys. 12 (2008) 981 [hep-th/0609129] [INSPIRE].

[56] C. Closset, H. Kim and B. Willett, Supersymmetric partition functions and the three-dimensional A-twist, JHEP 03 (2017) 074 [arXiv:1701.03171] [INSPIRE].

[57] M.F. Atiyah, Elliptic operators and compact groups, Lect. Notes Math. 401 (1974) 1.

[58] J. Quine, S. Heydari and R. Song, Zeta regularized products, Trans. Amer. Math. Soc. 338 (1993) 213.

[59] C. Closset, T.T. Dumitrescu, G. Festuccia and Z. Komargodski, The geometry of supersymmetric partition functions, JHEP 01 (2014) 124 [arXiv:1309.5876] [INSPIRE].

[60] B. Willett, Localization on three-dimensional manifolds, J. Phys. A 50 (2017) 443006 [arXiv: 1608.02958] [INSPIRE].

[61] F. Benini and A. Zaffaroni, A topologically twisted index for three-dimensional supersymmetric theories, JHEP 07 (2015) 127 [arXiv:1504.03698] [INSPIRE]. 
[62] J. Schmude, Localisation on Sasaki-Einstein manifolds from holomorphic functions on the cone, JHEP 01 (2015) 119 [arXiv:1401.3266] [INSPIRE].

[63] D. Baraglia and P. Hekmati, Moduli spaces of contact instantons, Adv. Math. 294 (2016) 562 [arXiv:1401.5140] [INSPIRE].

[64] C. Closset, H. Kim and B. Willett, Seifert fibering operators in $3 d \mathcal{N}=2$ theories, JHEP 11 (2018) 004 [arXiv: 1807.02328] [INSPIRE].

[65] A. Bawane, G. Bonelli, M. Ronzani and A. Tanzini, $\mathcal{N}=2$ supersymmetric gauge theories on $S^{2} \times S^{2}$ and Liouville gravity, JHEP 07 (2015) 054 [arXiv:1411.2762] [INSPIRE].

[66] N. Banerjee, B. de Wit and S. Katmadas, The off-shell 4D/5D connection, JHEP 03 (2012) 061 [arXiv:1112.5371] [INSPIRE].

[67] M. Honda and Y. Yoshida, Supersymmetric index on $T^{2} \times S^{2}$ and elliptic genus, arXiv: 1504.04355 [INSPIRE].

[68] A. Gadde, S.S. Razamat and B. Willett, On the reduction of $4 d \mathcal{N}=1$ theories on $\mathbb{S}^{2}, J H E P$ 11 (2015) 163 [arXiv:1506.08795] [INSPIRE].

[69] F. Benini, T. Nishioka and M. Yamazaki, $4 d$ index to $3 d$ index and $2 d$ TQFT, Phys. Rev. D 86 (2012) 065015 [arXiv:1109.0283] [INSPIRE].

[70] F. Benini, R. Eager, K. Hori and Y. Tachikawa, Elliptic genera of $2 d \mathcal{N}=2$ gauge theories, Commun. Math. Phys. 333 (2015) 1241 [arXiv:1308.4896] [INSPIRE].

[71] J.G. Russo, A Note on perturbation series in supersymmetric gauge theories, JHEP 06 (2012) 038 [arXiv:1203.5061] [INSPIRE].

[72] M. Honda, Borel summability of perturbative series in $4 D N=2$ and $5 D N=1$ supersymmetric theories, Phys. Rev. Lett. 116 (2016) 211601 [arXiv:1603.06207] [INSPIRE].

[73] M. Honda, How to resum perturbative series in $3 d N=2$ Chern-Simons matter theories, Phys. Rev. D 94 (2016) 025039 [arXiv: 1604.08653] [INSPIRE].

[74] H.-C. Kim, S.-S. Kim and K. Lee, 5-dim superconformal index with enhanced $E_{n}$ global symmetry, JHEP 10 (2012) 142 [arXiv:1206.6781] [INSPIRE].

[75] S. Terashima, Supersymmetric gauge theories on $S^{4} \times S^{1}$, Phys. Rev. D 89 (2014) 125001 [arXiv:1207.2163] [INSPIRE].

[76] H.-C. Kim, S. Kim, S.-S. Kim and K. Lee, The general M5-brane superconformal index, arXiv: 1307.7660 [INSPIRE].

[77] J. Kallen, J.A. Minahan, A. Nedelin and M. Zabzine, $N^{3}$-behavior from $5 D$ Yang-Mills theory, JHEP 10 (2012) 184 [arXiv:1207.3763] [INSPIRE].

[78] G. Giasemidis, R.J. Szabo and M. Tierz, Supersymmetric gauge theories, Coulomb gases and Chern-Simons matrix models, Phys. Rev. D 89 (2014) 025016 [arXiv:1310.3122] [InSPIRE].

[79] M. Mezei, S.S. Pufu and Y. Wang, Chern-Simons theory from M5-branes and calibrated M2-branes, JHEP 08 (2019) 165 [arXiv:1812.07572] [INSPIRE].

[80] Y. Dolivet and M. Tierz, Chern-Simons matrix models and Stieltjes-Wigert polynomials, J. Math. Phys. 48 (2007) 023507 [hep-th/0609167] [INSPIRE].

[81] M. Mariño, Chern-Simons theory, matrix integrals and perturbative three manifold invariants, Commun. Math. Phys. 253 (2004) 25 [hep-th/0207096] [INSPIRE]. 
[82] A. Brini, L. Griguolo, D. Seminara and A. Tanzini, Chern-Simons theory on L $(p, q)$ lens spaces and Gopakumar-Vafa duality, J. Geom. Phys. 60 (2010) 417 [arXiv:0809.1610] [INSPIRE].

[83] R.J. Szabo and M. Tierz, q-deformations of two-dimensional Yang-Mills theory: classification, categorification and refinement, Nucl. Phys. B 876 (2013) 234 [arXiv:1305.1580] [INSPIRE].

[84] J.A. Minahan and M. Zabzine, Gauge theories with 16 supersymmetries on spheres, JHEP 03 (2015) 155 [arXiv: 1502.07154] [INSPIRE].

[85] K. Polydorou, A. Rocén and M. Zabzine, $7 D$ supersymmetric Yang-Mills on curved manifolds, JHEP 12 (2017) 152 [arXiv:1710.09653] [INSPIRE].

[86] A. Rocén, 7D supersymmetric Yang-Mills on a 3-Sasakian manifold, JHEP 11 (2018) 024 [arXiv: 1808.06917] [INSPIRE].

[87] T. Friedrich and I. Kath, Einstein manifolds of dimension five with small first eigenvalue of the Dirac operator, J. Diff. Geom. 29 (1989) 263.

[88] J.P. Gauntlett, D. Martelli, J. Sparks and D. Waldram, Sasaki-Einstein metrics on $S^{2} \times S^{3}$, Adv. Theor. Math. Phys. 8 (2004) 711 [hep-th/0403002] [InSPIRE].

[89] J.C. Geipel, O. Lechtenfeld, A.D. Popov and R.J. Szabo, Sasakian quiver gauge theories and instantons on the conifold, Nucl. Phys. B 907 (2016) 445 [arXiv: 1601.05719] [InSPIRE]. 